\title{
ERGODICITY FOR STOCHASTIC POROUS MEDIA EQUATIONS WITH MULTIPLICATIVE NOISE*
}

\author{
KONSTANTINOS DAREIOTIS ${ }^{\dagger}$, BENJAMIN GESS $^{\ddagger}$, AND PAVLOS TSATSOULIS TS $^{-}$
}

\begin{abstract}
The long time behavior of solutions to stochastic porous media equations with nonlinear multiplicative noise on bounded domains with Dirichlet boundary data is studied. Based on weighted $L^{1}$-estimates, the existence and uniqueness of invariant measures with optimal bounds on the rate of mixing are proved. Along the way, the existence and uniqueness of entropy solutions are shown.
\end{abstract} rates

Key words. stochastic porous media, entropy solutions, invariant measures, optimal mixing

AMS subject classifications. 37A25, 76S99

DOI. $10.1137 / 19 \mathrm{M} 1278521$

1. Introduction. In this work we prove the existence and uniqueness of invariant measures, with optimal estimates on the rate of mixing, for stochastic porous media equations with nonlinear multiplicative noise

$$
\left\{\begin{array}{l}
\partial_{t} u(t, x)=\Delta\left(|u|^{m-1} u\right)(t, x)+\sum_{k=1}^{\infty} \sigma^{k}(x, u(t, x)) \dot{\beta}^{k}(t) \\
u(0)=\xi \\
\left.u\right|_{\partial Q}=0
\end{array}\right.
$$

on bounded domains $Q \subseteq \mathbb{R}^{d}$, where $\left(\beta^{k}\right)_{k \geq 1}$ is a sequence of independent Brownian motions, $m \in(1, \infty), \xi$ is the initial condition (which lies in a suitably weighted $L_{x}^{1}$ space), and $\left(\sigma^{k}\right)_{k \geq 1}$ is a sequence of Hölder continuous coefficients (for the exact assumptions, see sections 2 and 3.2). Our main result is the following contraction estimate (see Theorem 3.8 below): There exists a (uniform in the initial condition) constant $C>0$ such that for each pair of entropy solutions $u(\cdot ; \xi), u(\cdot ; \tilde{\xi})$ to $(1.1)$ with initial conditions $\xi, \tilde{\xi}$, respectively, we have

$$
\mathbb{E}\|u(t ; \xi)-u(t ; \tilde{\xi})\|_{L_{w ; x}^{1}} \leq C t^{-\frac{1}{m-1}}, t>0
$$

where $L_{w ; x}^{1}$ is the weighted $L_{x}^{1}$ space with weight $w$ given by the solution to $\Delta w=-1$ with zero Dirichlet boundary conditions on $Q$. This contraction estimate implies the existence and uniqueness of an invariant measure $\mu$ to (1.1) and the following optimal

\footnotetext{
* Received by the editors August 1, 2019; accepted for publication (in revised form) May 26, 2020; published electronically September 28, 2020.

https://doi.org/10.1137/19M1278521

Funding: The work of the authors was supported by the DFG grant CRC 1283 "Taming uncertainty and profiting from randomness and low regularity in analysis, stochastics and their applications."

${ }^{\dagger}$ School of Mathematics, University of Leeds, LS2 9JT Leeds, UK (K.Dareiotis@leeds.ac.uk).

${ }^{\ddagger}$ Max-Planck-Institut für Mathematik in den Naturwissenschaften, 04103 Leipzig, Germany, and Faculty of Mathematics, University of Bielefeld, 33615 Bielefeld, Germany (benjamin. gess@mis.mpg.de).

$\S$ Max-Planck-Institut für Mathematik in den Naturwissenschaften, 04103 Leipzig, Germany (pavlos.tsatsoulis@mis.mpg.de).
} 
bound (see Remark 3.13) on the rate of mixing:

$$
\sup _{\xi \in L_{w ; x}^{1}\|F\|_{\operatorname{Lip}\left(L_{w ; x}^{1}\right)}^{1} \leq 1} \sup _{t}\left|P_{t} F(\xi)-\int_{L_{w ; x}^{1}} F(\tilde{\xi}) \mu(\mathrm{d} \tilde{\xi})\right| \leq C t^{-\frac{1}{m-1}}, t>0,
$$

where $\operatorname{Lip}\left(L_{w ; x}^{1}\right)$ denotes the space of Lipschitz continuous functions from $L_{w ; x}^{1}$ to $\mathbb{R}$, and $P_{t}$ denotes the Markov semigroup on $B_{b}\left(L_{w ; x}^{1}\right)$ associated to (1.1) (see Theorem 3.12 below). On the way to these results we further prove the existence, uniqueness, and stability of entropy solutions to (1.1), and the time continuity of entropy solutions with values in $L_{\omega}^{1} L_{w ; x}^{1}$ (see Theorems 3.1, 3.2, and 3.3 below).

The existence, uniqueness, and mixing properties of solutions to stochastic porous media equations have attracted considerable attention in the literature (see section 1.1 below). However, all available results are essentially restricted to additive noise in the following sense: They either treat only purely additive noise or assume that the noise contains a sufficiently nondegenerate additive part. Therefore, (1.1) is out beyond the reach of existing results.

Stochastic porous media equations of the type (1.1) informally appear as continuum limits of interacting branching particle processes. More precisely, Méléard and Roelly have shown in [MR93] that, under appropriate rescaling, the mean field limit of branching particle processes that interact through a potential $V$ solves a nonlocal, nonlinear stochastic diffusion equation of the type

$$
\partial_{t} u(t, x)=\frac{1}{2} \Delta(|u|(V * u))(t, x)+\sqrt{b(x, u(t, x)) u(t, x)} \dot{W}(t),
$$

where $\dot{W}(t)$ is space-time white noise. Informally, localizing the particle interactions, by taking $V$ to a Dirac mass, leads to a stochastic partial differential equation (SPDE) of the type (1.1), albeit with space-time white noise. This last step has been rigorously justified in the deterministic case by Lions and Mas-Gallic [LMG01] and Carrillo, Craig, and Patacchini [CCP19]. We emphasize that (1.3) has purely multiplicative noise, without nondegenerate additive part, so that established methods on ergodicity and mixing do not apply.

In a broader scope, the aim of the present article is to understand the applicability of the dissipativity approach (see [DPZ14, section 11.6] and the references therein) to the ergodicity of SPDEs with multiplicative noise. While the focus is on stochastic porous media equations, the ideas are equally relevant for the case of semilinear SPDEs (see Remark 1.1 below). Let us briefly and informally recall the dissipativity approach in the case of additive noise; that is, let $u, \tilde{u}$ be solutions to

$$
\partial_{t} u(t, x)=\Delta\left(|u|^{m-1} u\right)(t, x)+G \dot{W}(t),
$$

with zero Dirichlet boundary conditions on $Q$ and diffusion coefficients $G$. Then, informally, using Lemma B.1 below we have

$$
\begin{aligned}
\partial_{t}\|u-\tilde{u}\|_{H_{x}^{-1}}^{2} & =2\left(\Delta\left(|u|^{m-1} u\right)-\Delta\left(|\tilde{u}|^{m-1} \tilde{u}\right), u-\tilde{u}\right)_{H_{x}^{-1}} \\
& =-2\left(|u|^{m-1} u-|\tilde{u}|^{m-1} \tilde{u}, u-\tilde{u}\right)_{L_{x}^{2}} \\
& \leq-2 C_{m}\|u-\tilde{u}\|_{L_{x}^{m+1}}^{m+1} \leq-2 C_{m}\|u-\tilde{u}\|_{H_{x}^{-1}}^{m+1},
\end{aligned}
$$

where $H_{x}^{-1}:=\left(H_{0 ; x}^{1}\right)^{*}$ and $C_{m}>0$. This implies contraction estimates of the type

$$
\|u(t)-\tilde{u}(t)\|_{H_{x}^{-1}}^{2} \leq C_{m} t^{-\frac{1}{m-1}}, \quad t>0
$$


and polynomial rates of mixing

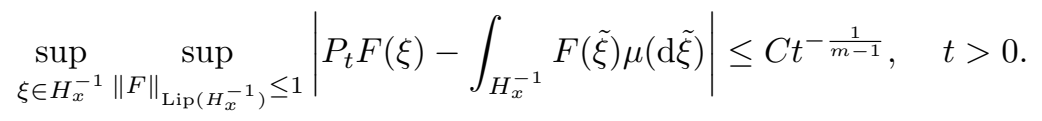

This argument is restricted to additive noise. Indeed, if we consider (1.1) then, following the previous computations, we have

$$
\partial_{t} \mathbb{E}\|u-\tilde{u}\|_{H_{x}^{-1}}^{2} \leq-2 C_{m} \mathbb{E}\|u-\tilde{u}\|_{H_{x}^{-1}}^{m+1}+\sum_{k=1}^{\infty} \mathbb{E}\left\|\sigma^{k}(u)-\sigma^{k}(\tilde{u})\right\|_{H_{x}^{-1}}^{2} .
$$

Even when $u \mapsto \sigma^{k}(\cdot, u)$ is Lipschitz continuous in $H_{x}^{-1}$ (which is rarely the case; see, for example, the discussion in [DGG19]), it is unclear how to prove stability without a smallness assumption on the Lipschitz constant of the coefficients $\sigma^{k}$.

The main insight of the present work is that the weighted topology $L_{w ; x}^{1}$ introduced here is better adapted to the dissipativity approach for SPDEs with multiplicative noise. In fact, it is shown that the stochastically perturbed equation enjoys the same stability properties as those of the deterministic PDE when considered in this weighted topology.

Remark 1.1. The same questions can be asked in the case of semilinear SPDEs, such as

$$
\partial_{t} u(t, x)=(\Delta u(t, x)+f(u(t, x)))+\sum_{k=1}^{\infty} \sigma^{k}(x, u(t, x)) \dot{\beta}^{k}(t),
$$

with zero Dirichlet boundary conditions. For simplicity let us assume that $f: \mathbb{R} \rightarrow \mathbb{R}$ is nondecreasing. Again, an $L^{2}$-based approach suffers from the Itô-correction terms, since, informally,

$$
\begin{aligned}
\partial_{t} \mathbb{E}\|u-\tilde{u}\|_{L_{x}^{2}}^{2} & \\
& =2 \mathbb{E}(\Delta(u-\tilde{u})+f(u)-f(\tilde{u}), u-\tilde{u})_{L_{x}^{2}}+\sum_{k=1}^{\infty} \mathbb{E}\left\|\sigma^{k}(u)-\sigma^{k}(\tilde{u})\right\|_{L_{x}^{2}}^{2} \\
& =-2 \mathbb{E}\|\nabla(u-\tilde{u})\|_{L_{x}^{2}}^{2}+2 \mathbb{E}(f(u)-f(\tilde{u}), u-\tilde{u})_{L_{x}^{2}}+\sum_{k=1}^{\infty}\left\|\sigma^{k}(u)-\sigma^{k}(\tilde{u})\right\|_{L_{x}^{2}}^{2} \\
& \leq-2 C_{P} \mathbb{E}\|u-\tilde{u}\|_{L_{x}^{2}}^{2}+\sum_{k=1}^{\infty}\left\|\sigma^{k}(u)-\sigma^{k}(\tilde{u})\right\|_{L_{x}^{2}}^{2}
\end{aligned}
$$

where $C_{P}$ is the Poincaré constant. This implies stability only if the Lipschitz constant of $u \mapsto \sigma^{k}(\cdot, u)$ is small enough. Hence, even if the deterministic PDE is stable, this is not necessarily inherited by the stochastically perturbed equation.

However, the weighted norm $\|\cdot\|_{L_{w ; x}^{1}}$ is better adapted to studying the stability 
of (1.5), in the sense that, informally,

$$
\begin{aligned}
\partial_{t} \mathbb{E}\|u-\tilde{u}\|_{L_{w ; x}^{1}}= & \mathbb{E} \int_{Q} \operatorname{sgn}(u-\tilde{u}) \Delta(u-\tilde{u}) w \mathrm{~d} x+\mathbb{E} \int_{Q} \operatorname{sgn}(u-\tilde{u})(f(u)-f(\tilde{u})) w \mathrm{~d} x \\
& +\frac{1}{2} \mathbb{E} \int_{Q} \operatorname{sgn}^{\prime}(u-\tilde{u}) \sum_{k=1}^{\infty}\left|\sigma^{k}(u)-\sigma^{k}(\tilde{u})\right|^{2} w \mathrm{~d} x \\
\leq & -\mathbb{E} \int_{Q} \nabla|u-\tilde{u}| \nabla w \mathrm{~d} x-\mathbb{E} \int_{Q} \operatorname{sgn}^{\prime}(u-\tilde{u})|\nabla(u-\tilde{u})|^{2} w \mathrm{~d} x \\
& +\mathbb{E} \int_{Q} \operatorname{sgn}(u-\tilde{u})(f(u)-f(\tilde{u})) w \mathrm{~d} x \\
+ & \frac{1}{2} \mathbb{E} \int_{Q} \operatorname{sgn}^{\prime}(u-\tilde{u}) \sum_{k=1}^{\infty}\left|\sigma^{k}(u)-\sigma^{k}(\tilde{u})\right|^{2} w \mathrm{~d} x .
\end{aligned}
$$

The second and third terms on the right-hand side of the last inequality are nonpositive, the fourth one vanishes under certain regularity assumptions on $\sigma$ (see Assumption 2.2), while for the first, we have that

$-\mathbb{E} \int_{Q} \nabla|u-\tilde{u}| \nabla w \mathrm{~d} x=\mathbb{E} \int_{Q}|u-\tilde{u}| \Delta w \mathrm{~d} x=-\mathbb{E} \int_{Q}|u-\tilde{u}| \mathrm{d} x \leq-C \mathbb{E} \int_{Q}|u-\tilde{u}| w \mathrm{~d} x$.

Consequently,

$$
\partial_{t} \mathbb{E}\|u-\tilde{u}\|_{L_{w ; x}^{1}} \leq-C \mathbb{E}\|u-\tilde{u}\|_{L_{w ; x}^{1}}
$$

which immediately implies exponential mixing, with the same rate as in the deterministic case.

While the above remark outlines the principal idea in a related setting, a rigorous justification of this informal argument is much more involved: Since the intended estimates rely on the weighted $L_{x}^{1}$ topology, the appropriate concept of solutions is that of entropy solutions. Indeed, the informal computations (1.6) rely on an Itô formula applied to the weighted $L_{w ; x}^{1}$-norm. Due to the degeneracy of (1.1), the corresponding solutions are expected to have limited regularity. The justification of Itô's formula in the context of low regularity solutions and irregular functions, such as the $L_{w ; x}^{1}$-norm, is the key idea of the concept of entropy solutions. This aspect is further complicated by working on a bounded domain. In fact, the uniqueness of entropy solutions for (1.1) on bounded domains could not previously be shown due to additional boundary terms. Also, this difficulty is overcome in the present work by the use of the weighted $L_{x}^{1}$ spaces instead. Another obstacle appears in the construction of the associated Markov semigroup to (1.1), due to the lack of $\mathbb{P}$-almost surely uniform in time estimates (cf., e.g., (3.1) below). In order to overcome this difficulty, we prove instead the time continuity of entropy solutions with values in $L_{\omega}^{1} L_{w ; x}^{1}$, which is sufficient to deduce the Markov property.

Finally, let us point out that the reason we work with Dirichlet boundary conditions on bounded domains is twofold: First, the principal approach outlined above relies on the existence of an appropriate weight $w$ which is a positive solution to the Dirichlet problem $\Delta w=-1$. In the case of periodic boundary data, the existence of such a weight or an appropriate replacement is not clear. Second, we extend the framework of entropy solutions in [DGG19] where the periodic case was considered. 
This extension is interesting in its own regard, as it is not immediate due to the presence of additional boundary terms, which in the current framework are treated by working in weighted $L^{1}$-spaces.

1.1. Comments on the literature. The available results can be categorized into two classes: The first class of results relies on the dissipativity approach exploiting the contractive properties of the deterministic porous media equation, while the second class relies on the mixing effects of the random perturbation. As such, these two classes lead to essentially different assumptions and results.

The first results on the existence of invariant measures for stochastic porous media equations were obtained by Da Prato and Röckner [DPR04a, DPR04b] and Bogachev, Da Prato and Röckner [BDPR04]. The dissipativity approach to proving the existence, uniqueness and rates of mixing was first applied to stochastic porous media equations by Da Prato et al. in [DPRRW06]; see also the more recent monograph by Barbu, Da Prato, and Röckner [BDPR16]. As explained above, this approach is restricted to additive noise. Since the obtained estimates are based on the contractive properties of the (deterministic) porous media operator, no nondegeneracy assumptions on the noise need to be made, and the obtained rates of mixing are of polynomial type. In [BGLR11] the dissipativity approach was further used by Beyn et al. in order to prove that the random attractor consists of a single random point. A generalization of the dissipativity approach, based on coupling arguments, has been introduced by Gess and Tölle in [GT14, GT16], allowing us to prove the ergodicity of generalized porous media equations in cases where no strict contraction estimates, such as (1), apply.

Concerning the second class of results, in the case of purely additive noise,

$$
\partial_{t} u(t, x)=\Delta\left(|u|^{m-1} u\right)(t, x)+G \dot{W}(t),
$$

couplings by change of measure were constructed by Wang in [Wan07, Wan13]; see also Liu [Liu09]. This construction relies on a nondegeneracy assumption on the diffusion coefficients $G$. In particular, it has to be assumed that $G$ is surjective onto the energy space $L_{x}^{m+1}$. Since this assumption competes with the smoothness assumption on the noise required by the well-posedness theory (cf., e.g., Liu and Röckner [LR15]), this restricts the applicability of these results to one spatial dimension. Because this approach exploits the nondegeneracy of the noise by means of establishing Harnack inequalities on the resulting Markov semigroup, the associated rate of mixing is of exponential type; that is, there is a unique invariant measure $\mu$ and constants $\lambda>0$, $C \geq 0$ such that

$$
\sup _{\xi}\left\|P_{t} \delta_{\xi}-\mu\right\|_{T V} \leq C e^{-\lambda t}, \quad t>0
$$

Lower bounds on the exponential rate $\lambda$ have been obtained by Wang in [Wan15a]. Flandoli, Gess, and Scheutzow in [FGS17] and Gess in [Ges13] used these methods in order to prove synchronization by noise, in the sense that the random attractor was shown to consist of a single random point. This line of argument has been further improved by Wang in [Wan15b], where coupling by change of measure has been replaced by reflection coupling, which allowed also the inclusion of perturbations by multiplicative noise, while retaining the nondegeneracy assumption on the additive noise part, that is,

$$
\partial_{t} u(t, x)=\Delta\left(|u|^{m-1} u\right)(t, x)+B(u) \dot{W}^{1}(t)+G \dot{W}^{2}(t),
$$


with $G$ being nondegenerate as above. This work seems to be the only previous result on ergodicity for stochastic porous media equations with multiplicative noise in the literature. However, in this work $B$ is still assumed to be Lipschitz continuous in $H_{x}^{-1}$, which in the case of $B(u)=\sigma(x, u(x))$ (as in (1.1)) essentially implies that $\sigma$ is linear in $u$.

In the case of linear multiplicative noise, the stochastic porous media equation can be transformed into a deterministic porous media equation with random coefficients. Under further, stringent assumptions on the noise, this can be used to derive statements on the long-time behavior. See, for example, [Lot07, Ges14, BR11, Ges15] and the references therein.

We conclude the discussion of available results on the ergodicity of stochastic porous media equations by emphasizing that there are no previous results on the ergodicity of (1.1) with purely nonlinear multiplicative noise, as it appears in (1.3).

The well-posedness of entropy solutions to (1.1) on the torus has been recently shown by Dareiotis, Gerencsér, and Gess in [DGG19]. In the context of the wellposedness of solutions, it already has been realized in [DGG19] that $L_{x}^{1}$ appears to be better suited than $H_{x}^{-1}$, which was used in many previous works (see, for example, [LR15] and the references therein), since the nonlinear diffusion coefficients behave nicely in $L_{x}^{1}$, while they are not expected to be even Lipschitz continuous in $H_{x}^{-1}$. The proof of well-posedness of entropy solutions to (1.1) on bounded domains, given in the present work, builds upon the analysis from [DGG19]. In contrast to the periodic case considered in [DGG19], the presence of the boundary introduces the need for a weighted $L^{1}$-norm in order to control boundary terms.

1.2. Organization of the article. In section 2 we set up the right formulation for studying the well-posedness of (1.1). In section 3 we present our main results.

In section 3.1 we discuss the well-posedness of stochastic porous media equations on bounded domains. We begin with the existence, uniqueness, and $L^{1}$-contraction of entropy solutions (see Theorem 3.1), stability with respect to the data (see Theorem 3.2 ), and continuity in $L_{\omega}^{1} L_{w ; x}^{1}$ (see Theorem 3.3). These results hold for more general porous media operators $\Delta A$ (see Assumption 2.1(a)), and the initial condition $\xi$ is assumed to be in $L_{\omega}^{m+1} L_{x}^{m+1}$. As a corollary of Theorems 3.1 and 3.3 we present an extension result to initial conditions in $L_{\omega}^{1} L_{w ; x}^{1}$ (see Proposition 3.4). In section 3.2 we present a bound for the solutions in $L_{\omega}^{m+1} L_{x}^{m+1}$ which is uniform in the initial condition and in time (see Proposition 3.6). We then discuss the main contraction estimate (see Theorem 3.8), the Markov property (see Proposition 3.10), and mixing to a unique equilibrium with optimal rate (see Theorem 3.12).

In section 4 we prove some important $L^{1}$-estimates, in section 5 we prove the results listed in section 3.1, and in section 6 we prove the results listed in section 3.2. Many technical results and proofs can be found in Appendix A.

1.3. Notation. For a set $S \subset \mathbb{R}^{d}$ and $m \in \mathbb{N}$, we denote by $C^{m}(S)$ the space of $m$-times differentiable functions on $S$ and by $C_{c}^{m}(S)$ the subset of $m$-times differentiable compactly supported functions on $S$. Given a variable $\mathfrak{s} \in S, p \in[1, \infty]$, and $m \in \mathbb{R}$, we denote by $L_{\mathfrak{s}}^{p}$ and $W_{\mathfrak{s}}^{m, p}$ the usual $L^{p}$ and $W^{m, p}$ spaces of functions in this variable. If $p=2$ we write $H_{\mathfrak{s}}^{m}$ instead of $W_{\mathfrak{s}}^{m, 2}$. If, in addition, $m \in \mathbb{N}$, we write $H_{0 ; \mathfrak{s}}^{m}$ for the closure of $C_{c}^{m}(S)$ in $H_{\mathfrak{s}}^{m}$. For a probability space $(\Omega, \mathcal{F}, \mathbb{P})$ and $p \in[1, \infty)$, we denote by $L_{\omega}^{p}$ the space of $p$-integrable random variables in $\omega \in \Omega$. If the probability space carries a filter $\left(\mathcal{F}_{t}\right)_{t \geq 0}$, we denote by $L_{\omega, t}^{p}$ the space of predictable $p$-integrable random processes in $(\omega, t) \in \Omega \times[0, T]$ for every $T>0$. For spaces of functions of several arguments, we sometimes use mixed notation. For example, $L_{\omega}^{p} L_{x}^{q}$ stands for 
the space of $p$-integrable random variables taking values in $L_{x}^{q}$, while $L_{\omega, t}^{p} L_{x}^{q}$ stands for the space of predictable $p$-integrable random processes taking values in $L_{x}^{q}$.

We denote by $w$ the solution to the boundary value problem

$$
\left\{\begin{array}{l}
\Delta w=-1 \\
\left.w\right|_{\partial Q}=0
\end{array}\right.
$$

It is well known that $w \in H_{0 ; x}^{1}$ and $w>0$ in $Q$. We define $L_{w ; x}^{1}$ to be the space of measurable functions $f: Q \rightarrow \mathbb{R}$ such that

$$
\|f\|_{L_{w ; x}^{1}}:=\int_{Q}|f(x)| w(x) \mathrm{d} x<\infty .
$$

We define the set

$$
\mathcal{S}:=\{d, K, m,|Q|, T\},
$$

where $d$ denotes the dimension of the $x$-space, $K$ and $m$ are positive constants given by Assumptions 2.1 and 2.2 below, $|Q|$ denotes the volume of $Q$, and $T>0$.

Throughout the article, $C$ denotes a strictly positive constant which depends on the structural set $\mathcal{S}$, unless otherwise stated, and might change from line to line. In the proofs we will frequently use the notation $a \lesssim b$ by which we mean $a \leq C b$. The notation $a \lesssim_{q} b$ (respectively, $C_{q}$ ) means that the constant $C$ depends on $\mathcal{S}$ and on $q$. We also write $a \vee b$ (respectively, $a \wedge b$ ) to denote the maximum (respectively, minimum) between $a$ and $b$.

In the proofs we sometimes use the abbreviation $\int_{x}, \int_{t}$ instead of $\int_{Q} \mathrm{~d} x, \int_{0}^{T} \mathrm{~d} t$. An analogous notation is used for multiple integrals. For example, $\int_{t, x}$ stands for $\int_{0}^{T} \int_{Q} \mathrm{~d} x \mathrm{~d} t$.

2. Formulation. We consider a generalization of (1.1) in the form

$$
\left\{\begin{array}{l}
\partial_{t} u(t, x)=\Delta A(u(t, x))+\sigma^{k}(x, u(t, x)) \dot{\beta}^{k}(t), \\
u(0)=\xi \\
\left.u\right|_{\partial Q}=0
\end{array}\right.
$$

under Assumptions 2.1 and 2.2 below. For simplicity, we also assume that the bounded domain $Q \subset \mathbb{R}^{d}$ is smooth, although this assumption can be relaxed. From now on we fix a filtered probability space $\left(\Omega, \mathcal{F},\left\{\mathcal{F}_{t}\right\}_{t \geq 0}, \mathbb{P}\right)$ with a sequence $\left(\beta^{k}\right)_{k \geq 1}$ of independent Brownian motions.

For a locally integrable function $f: \mathbb{R} \rightarrow \mathbb{R}$ we define

$$
[f](r):=\int_{0}^{r} f(\zeta) \mathrm{d} \zeta,[f, c](r):=\int_{c}^{r} f(\zeta) \mathrm{d} \zeta .
$$

We also let $\mathfrak{a}:=\sqrt{A^{\prime}}$.

Assumption 2.1. The following hold for some $K \geq 1$ and $m>1$.

(a) The function $A: \mathbb{R} \mapsto \mathbb{R}$ is differentiable, strictly increasing, and odd. The function $\mathfrak{a}$ is differentiable away from the origin, and it satisfies the bounds

$$
|\mathfrak{a}(0)| \leq K,\left|\mathfrak{a}^{\prime}(r)\right| \leq K|r|^{\frac{m-3}{2}} \quad \text { if } r>0
$$

Copyright $@$ ㅇ by SIAM. Unauthorized reproduction of this article is prohibited. 
as well as

$$
K \mathfrak{a}(r) \geq I_{|r| \geq 1}, K|[\mathfrak{a}](r)-[\mathfrak{a}](\tilde{r})| \geq \begin{cases}|r-\tilde{r}| & \text { if }|r| \vee|\tilde{r}| \geq 1 \\ |r-\tilde{r}|^{\frac{m+1}{2}} & \text { if }|r| \vee|\tilde{r}|<1\end{cases}
$$

(b) The initial condition $\xi$ is an $\mathcal{F}_{0}$-measurable $L_{x}^{m+1}$-valued random variable such that $\mathbb{E}\|\xi\|_{L_{x}^{m+1}}^{m+1}<\infty$.

Assumption 2.2. The function $\sigma: Q \times \mathbb{R} \mapsto \ell^{2}$ satisfies the following bounds. There exist $\kappa \in\left(0, \frac{1}{2}\right], \bar{\kappa} \in\left(\frac{1}{m \wedge 2}, 1\right]$, and $K \geq 1$ such that for every $r \in \mathbb{R}, \tilde{r} \in$ $[r-1, r+1]$, and $x, y \in Q$,

$$
|\sigma(x, r)|_{\ell^{2}} \leq K(1+|r|),|\sigma(x, r)-\sigma(y, \tilde{r})|_{\ell^{2}} \leq K|r-\tilde{r}|^{\frac{1}{2}+\kappa}+K(1+|r|)|x-y|^{\bar{\kappa}} .
$$

For $\sigma, \tilde{\sigma}: Q \times \mathbb{R} \mapsto \ell^{2}$ satisfying Assumption 2.2 we set

$$
d(\sigma, \tilde{\sigma}):=\sup _{x \in Q, r \in \mathbb{R}} \frac{|\sigma(x, r)-\tilde{\sigma}(x, r)|_{\ell^{2}}^{2}}{(1+|r|)^{m+1}} .
$$

Definition 2.3. A pair of functions $(\eta, \phi)$ is called admissible if

(i) $\eta \in C^{2}(\mathbb{R}), \eta^{\prime \prime} \geq 0$, and $\operatorname{supp} \eta^{\prime \prime}$ is compact; and

(ii) $\phi \geq 0, \phi=\varphi \varrho$ where $\varphi \in C_{c}^{\infty}([0, T))$ and $\varrho \in C_{c}^{\infty}(Q)$. (2.1).

Similarly to [DGG19] we give the following definition of entropy solutions for

Definition 2.4. A predictable stochastic process $u: \Omega \times[0, \infty) \rightarrow L_{x}^{m+1}$ is an entropy solution to (2.1) if

i. $u \in L_{\omega, t}^{m+1} L_{x}^{m+1}$ and $A(u) \in L_{\omega, t}^{2} H_{0 ; x}^{1}$;

ii. for every $f \in C(\mathbb{R})$ bounded we have $[\mathfrak{a} f](u) \in L_{\omega, t}^{2} H_{x}^{1}$ and $\partial_{x_{i}}[\mathfrak{a} f](u)=f(u) \partial_{x_{i}}[\mathfrak{a}](u)$; and

iii. for every admissible pair of functions $(\eta, \phi)$ as in Definition 2.3 we have that

$$
\begin{aligned}
-\int_{0}^{T} \int_{Q} \eta(u) \partial_{t} \phi \mathrm{d} x \mathrm{~d} t \leq & \int_{Q} \eta(\xi) \phi(0) \mathrm{d} x+\int_{0}^{T} \int_{Q}\left[\eta^{\prime} \mathfrak{a}^{2}\right](u) \Delta \phi \mathrm{d} x \mathrm{~d} t \\
& +\int_{0}^{T} \int_{Q}\left(\frac{1}{2} \phi \eta^{\prime \prime}(u)|\sigma(u)|_{\ell^{2}}^{2}-\phi \eta^{\prime \prime}(u)|\nabla[\mathfrak{a}](u)|^{2}\right) \mathrm{d} x \mathrm{~d} t \\
& +\int_{0}^{T} \int_{Q} \phi \eta^{\prime}(u) \sigma^{k}(u) \mathrm{d} x \mathrm{~d} \beta^{k}(t) .
\end{aligned}
$$

We refer to $(2.1)$ as $\mathcal{E}(A, \sigma, \xi)$. In what follows we write $u(\cdot ; \xi)$ to denote the solution of $\mathcal{E}(A, \sigma, \xi)$. If the value of the initial condition is clear from the context, we simply write $u$.

\section{Main results.}

3.1. Well-posedness. The next two theorems build upon the analysis from [DGG19] and concern the existence, uniqueness and stability of entropy solutions to (2.1). All of the results in this subsection hold for fixed, but arbitrarily large, $T>0$.

TheOREm 3.1. Let Assumptions 2.1 and 2.2 hold. Then, there exists a unique entropy solution u of $\mathcal{E}(A, \sigma, \xi)$. Moreover, if $u(t ; \tilde{\xi})$ is an entropy solution of $\mathcal{E}(A, \sigma, \tilde{\xi})$, the following contraction estimate holds:

$$
\sup _{t \in[0, T]} \operatorname{ess} \mathbb{E}\|u(t ; \xi)-u(t ; \tilde{\xi})\|_{L_{w ; x}^{1}} \leq \mathbb{E}\|\xi-\tilde{\xi}\|_{L_{w ; x}^{1}} .
$$

Copyright $@$ by SIAM. Unauthorized reproduction of this article is prohibited. 
TheOREM 3.2. Let $\left(A_{n}\right)_{n \geq 1}$ and $\left(\xi_{n}\right)_{n \geq 1}$ satisfy Assumption 2.1, and let $\left(\sigma_{n}\right)_{n \geq 1}$ satisfy Assumption 2.2, uniformly in $n$. Assume furthermore that for $n \rightarrow \infty, A_{n} \rightarrow$ $A$ uniformly on compact sets, $\xi_{n} \rightarrow \xi$ in $L_{\omega}^{m+1} L_{x}^{m+1}$, and $d\left(\sigma_{n}, \sigma\right) \rightarrow 0$ for some $A$ and $\xi$ satisfying Assumption 2.1 and $\sigma$ satisfying Assumption 2.2. Let $u_{n}$, $u$ be the unique entropy solutions of $\mathcal{E}\left(A_{n}, \sigma_{n}, \xi_{n}\right), \mathcal{E}(A, \sigma, \xi)$. Then, as $n \rightarrow \infty, u_{n} \rightarrow u$ in $L_{\omega, t}^{1} L_{w ; x}^{1}$.

In the next theorem we prove the continuity of entropy solutions in $L_{\omega}^{1} L_{w ; x}^{1}$.

TheOREm 3.3. Let Assumptions 2.1 and 2.2 hold. If $u$ is an entropy solution of $\mathcal{E}(A, \sigma, \xi)$, then $u \in C\left([0, T] ; L_{\omega}^{1} L_{w ; x}^{1}\right)$.

As a corollary of the contraction estimate (3.1) and the continuity in $L_{\omega}^{1} L_{w ; x}^{1}$ we have the following extension result.

Proposition 3.4. Let Assumptions 2.1 and 2.2 hold. The mapping

$$
L_{\omega}^{m+1} L_{x}^{m+1} \ni \xi \mapsto u(\cdot ; \xi) \in C\left([0, T] ; L_{\omega}^{1} L_{w ; x}^{1}\right)
$$

extends uniquely to a continuous map from $L_{\omega}^{1} L_{w ; x}^{1}$ to $C\left([0, T] ; L_{\omega}^{1} L_{w ; x}^{1}\right)$. Furthermore, the following contraction estimate holds for every $\xi, \tilde{\xi} \in L_{\omega}^{1} L_{w ; x}^{1}$ :

$$
\sup _{t \in[0, T]} \mathbb{E}\|u(t ; \xi)-u(t ; \tilde{\xi})\|_{L_{w ; x}^{1}} \leq \mathbb{E}\|\xi-\tilde{\xi}\|_{L_{w ; x}^{1}} .
$$

3.2. Ergodicity. In this section we assume that $A(r)=|r|^{m-1} r$ for $m \in(1, \infty)$. From now on, it will be more convenient to work with initial conditions $\xi \in L_{w ; x}^{1}$. In the following, we define $u(\cdot ; \xi)$ for $\xi \in L_{w ; x}^{1}$ by continuity using Proposition 3.4. By Remark 3.5 and Proposition 3.6 below, one can prove that for every $\xi \in L_{\omega}^{1} L_{w ; x}^{1}$ the extension $u(\cdot ; \xi)$ is an entropy solution of $(2.1)$ on $(0, T]$ for every $T>0$, in the sense that it satisfies Definition 2.4 with $[0, T]$ replaced by $[s, T]$, for every $s \in(0, T]$.

Remark 3.5. Although Proposition 3.4 allows us to extend $u(\cdot ; \xi)$ for $\xi \in L_{\omega}^{1} L_{w ; x}^{1}$ by continuity, it is unclear whether the extension solves (2.1) in general. However, it is easy to see that if there exists a sequence $\xi_{n} \rightarrow \xi$ such that for every $s>0$

$$
\sup _{n \geq 1} \sup _{t \in[s, T]} \mathbb{E}\left\|u\left(t ; \xi_{n}\right)\right\|_{L_{x}^{m+1}}<\infty,
$$

then $u(\cdot ; \xi)$ is an entropy solution of $(2.1)$ on $(0, T]$. Indeed, since $u\left(\cdot ; \xi_{n}\right) \rightarrow u(\cdot ; \xi)$ in $C\left([0, T] ; L_{\omega}^{1} L_{w ; x}^{1}\right)$, we know that for every $s \in(0, T]$, passing to a subsequence, $u\left(s, x ; \xi_{n}\right) \rightarrow u(s, x ; \xi)$ for almost every $(\omega, x)$. Hence, by Fatou's lemma we have

$$
\mathbb{E}\|u(s ; \xi)\|_{L_{x}^{m+1}} \leq \liminf _{n \rightarrow \infty} \mathbb{E}\left\|u\left(s ; \xi_{n}\right)\right\|_{L_{x}^{m+1}} \leq \sup _{n \geq 1} \mathbb{E}\left\|u\left(s ; \xi_{n}\right)\right\|_{L_{x}^{m+1}}
$$

and the latter quantity is uniformly bounded in $n$ due to (3.3). Hence, by Theorem 3.1 there exists a unique solution of $(2.1)$ on $[s, T]$ with initial condition $u(s ; \xi)$, which we denote by $u_{s}(\cdot ; u(s ; \xi))$. By Corollary 6.1 we know that $u\left(\cdot ; \xi_{n}\right)$ coincides with $u_{s}\left(\cdot ; u\left(s ; \xi_{n}\right)\right)$ (the unique entropy solution of $(2.1)$ with initial condition $\left.u\left(s ; \xi_{n}\right)\right)$ on $[s, T]$. Since $u\left(s ; \xi_{n}\right) \rightarrow u(s ; \xi)$ in $L_{\omega}^{1} L_{w ; x}^{1}$, using (3.2) we see that $u_{s}\left(\cdot ; u\left(s ; \xi_{n}\right)\right) \rightarrow$ $u_{s}(\cdot ; u(s ; \xi))$ in $C\left([s, T] ; L_{\omega}^{1} L_{w ; x}^{1}\right)$. But $u\left(t ; \xi_{n}\right)=u_{s}\left(t ; u\left(s ; \xi_{n}\right)\right)$ for $t \in[s, T]$, which, in turn, implies that $u(t ; \xi)=u_{s}(t ; u(s ; \xi))$. Since $s \in(0, T]$ is arbitrary, this proves that $u(\cdot ; \xi)$ is an entropy solution on $(0, T]$.

The next proposition states that entropy solutions satisfy the so-called "coming down from infinity" property, which implies (3.3).

Copyright (c) by SIAM. Unauthorized reproduction of this article is prohibited. 
Proposition 3.6. Let Assumption 2.2 hold. Then,

$$
\sup _{\xi \in L_{\omega, x}^{m+1}} \sup _{t \in[0, \infty)}(t \wedge 1)^{\frac{m+1}{m-1}} \mathbb{E}\|u(t ; \xi)\|_{L_{x}^{m+1}}^{m+1}<\infty .
$$

Remark 3.7. By a simple application of Fatou's lemma we can replace the supremum over $\xi \in L_{\omega, x}^{m+1}$ by a supremum over $\xi \in L_{\omega}^{1} L_{w ; x}^{1}$ in (3.4).

The choice $A(r)=|r|^{m-1} r$ allows us to obtain a polynomial (with respect to time) decay for differences of entropy solutions uniformly in the initial conditions, as shown in the next theorem.

Theorem 3.8. Let Assumption 2.2 hold, and let $m_{*}=\frac{m}{m-1}$. There exists $C>0$ depending only on $m$ such that for all $t \geq 0$ we have

$$
\sup _{\xi, \tilde{\xi} \in L_{\omega}^{1} L_{w ; x}^{1}} \mathbb{E}\|u(t ; \xi)-u(t, \tilde{\xi})\|_{L_{w ; x}^{1}} \leq C\|w\|_{L_{x}^{m *}}^{m_{*}} t^{-\frac{1}{m-1}} .
$$

Below we let $B_{b}\left(L_{w ; x}^{1}\right)$ be the space of bounded Borel measurable functions from $L_{w ; x}^{1}$ to $\mathbb{R}$. We need the following definition.

Definition 3.9. We define $P_{t}: B_{b}\left(L_{w ; x}^{1}\right) \rightarrow B_{b}\left(L_{w ; x}^{1}\right)$ by

$$
P_{t} F(\xi):=\mathbb{E} F(u(t ; \xi)),
$$

where $F \in B_{b}\left(L_{w ; x}^{1}\right)$ and $\xi \in L_{w ; x}^{1}$.

Proposition 3.10. Let Assumption 2.2 hold. The family $\left(P_{t}\right)_{t \geq 0}$ is a Feller Markov semigroup on $B_{b}\left(L_{w ; x}^{1}\right)$.

Remark 3.11. One can actually define $P_{t}: B_{b}\left(L_{w ; x}^{1}\right) \rightarrow B_{b}\left(L_{w ; x}^{1}\right)$ for any $A$ satisfying Assumption 2.1(a) using Proposition 3.4. It is clear from the proof of Proposition 3.10 that $P_{t}$ is a Feller Markov semigroup on $B_{b}\left(L_{w ; x}^{1}\right)$ even in this case.

Theorem 3.8 provides a quantitative estimate for the semigroup $P_{t}$ acting on Lipschitz continuous functions on $L_{w ; x}^{1}$ and allows us to prove the existence and uniqueness of an invariant measure (which is actually supported on $L_{x}^{m+1}$ ). It also provides optimal mixing rates (see Remark 3.13) uniformly in the initial condition. We summarize in the following theorem.

Theorem 3.12. Let Assumption 2.2 hold, and let $m_{*}=\frac{m}{m-1}$. There exists a unique invariant measure $\mu \in \mathcal{M}_{1}\left(L_{w ; x}^{1}\right)$ for the semigroup $P_{t}$ which, moreover, is supported on $L_{x}^{m+1}$. Furthermore, there exists $C>0$, depending only on $m$, such that for all $t \geq 0$,

$$
\sup _{\xi \in L_{w ; x}^{1}\|F\|_{\operatorname{Lip}\left(L_{w ; x}^{1}\right)}^{1} \leq 1} \sup _{t}\left|P_{t} F(\xi)-\int_{L_{w ; x}^{1}} F(\tilde{\xi}) \mu(\mathrm{d} \tilde{\xi})\right| \leq C\|w\|_{L_{x}^{m *}}^{m_{*}^{*}} t^{-\frac{1}{m-1}},
$$

where $\operatorname{Lip}\left(L_{w ; x}^{1}\right)$ is the space of Lipschitz continuous functions from $L_{w ; x}^{1}$ to $\mathbb{R}$.

Remark 3.13. The decay rate in Theorems 3.8 and 3.12 is optimal, in the sense that there exists a solution to the deterministic system that cannot decay faster than $t^{-\frac{1}{m-1}}$ as $t \rightarrow \infty$. Indeed, one can consider the homogeneous porous media equation $\partial_{t} u=\Delta\left(|u|^{m-1} u\right)$ with Dirichlet boundary conditions and search for solutions of the 
form $u(t, x)=(1+t)^{-\frac{1}{m-1}} f(x)$. It is easy to check that $u$ is a solution if $f$ satisfies the following equation:

$$
\left\{\begin{array}{l}
\Delta\left(|f|^{m-1} f\right)+\frac{1}{m-1} f=0 \\
\left.f\right|_{\partial Q}=0
\end{array}\right.
$$

Existence and regularity of nonzero solutions to (3.5) for sufficiently smooth bounded domains was studied in [AP81]. In particular, [AP81, Proposition 1] implies the existence of a nonzero solution $f$ which is bounded on $\partial \Omega \cup \Omega$.

We would like to point out that in the case of additive noise, under sufficient nondegeneracy assumptions, it is known that the decay is exponential in the total variational norm (see, for example, [Wan15a, section 3]).

4. The $(\star)$-property and $L^{1}$-estimates. In this section we introduce the $(\star)$ property, a purely technical concept, and derive the basic $L^{1}$-estimates which we use in later sections to prove our main results. Before we proceed we need some notation.

Below we fix a nonnegative compactly supported smooth function $\rho: \mathbb{R} \rightarrow \mathbb{R}$ supported in $(0,1)$ such that $\int \rho(t) \mathrm{d} t=1$, and for $\theta \in(0,1)$ we set $\rho_{\theta}(t):=\theta^{-1} \rho\left(\theta^{-1} t\right)$. For $x \in \mathbb{R}^{d}$ we also let $\varrho(x):=\prod_{i=1}^{d} \rho\left(x_{i}\right)$, and for $\varepsilon \in(0,1)$ we set $\varrho_{\varepsilon}(x):=$ $\varepsilon^{-d} \varrho\left(\varepsilon^{-1} x\right)$.

For $g \in C^{\infty}(\mathbb{R})$ with supp $g^{\prime}$ compact, $\tilde{\varrho} \in C_{c}^{\infty}(Q \times Q), \varphi \in C_{c}^{\infty}((0, T))$, $\tilde{\sigma}$ as in Assumption 2.2, predictable random variable $\tilde{u} \in L_{\omega, t}^{m+1} L_{x}^{m+1}, \theta>0$, and $a \in \mathbb{R}$, we set

$$
\begin{aligned}
& \phi_{\theta}(t, x, s, y):=\tilde{\varrho}(x, y) \rho_{\theta}(t-s) \varphi\left(\frac{t+s}{2}\right), \\
& F_{\theta}(t, x, a):=\int_{0}^{T} \int_{Q} \tilde{\sigma}^{k}(y, \tilde{u}(s, y)) g(\tilde{u}(s, y)-a) \phi_{\theta}(t, x, s, y) \mathrm{d} y \mathrm{~d} \beta^{k}(s),
\end{aligned}
$$

with a slight abuse of notation since we hide the dependence of $\phi_{\theta}$ and $F_{\theta}$ on the various functions.

We need the following definition.

Definition 4.1. We say that a predictable random variable $u \in L_{\omega, t}^{m+1} L_{x}^{m+1}$ has the ( $\star$ )-property with coefficient $\sigma$ if for every $g, \varrho, \varphi, \tilde{\sigma}, \tilde{u}$ as above and for every $\theta>0$ sufficiently small, we have that $F_{\theta}(\cdot, \cdot, u) \in L_{\omega}^{1} L_{t, x}^{1}$ and

$$
\begin{aligned}
& \mathbb{E} \int_{0}^{T} \int_{Q} F_{\theta}(t, x, u(t, x)) \mathrm{d} x \mathrm{~d} t \\
& \quad \leq-\mathbb{E} \int_{[0, T]^{2}} \int_{Q^{2}} \sigma^{k}(x, u(s, x)) \tilde{\sigma}^{k}(y, \tilde{u}(s, y)) g^{\prime}(u(s, x) \\
& \quad-\tilde{u}(s, y)) \phi_{\theta}(t, x, s, y) \mathrm{d} x \mathrm{~d} y \mathrm{~d} t \mathrm{~d} s+C \theta^{1-\mu}
\end{aligned}
$$

for $\mu=\frac{3 m+5}{4(m+1)}$ and some constant $C>0$ (independent of $\theta$ ).

The main result of this section is Lemma 4.4 which is the counterpart of [DGG19, Theorem 4.1] for Dirichlet boundary conditions. For the reader's convenience we split the proof of Lemma 4.4 into Proposition 4.2 and Lemma 4.3.

From now on, for $\alpha, \delta, \varepsilon \in(0,1)$ and $\lambda \geq 0$, we set

$$
\mathcal{G}_{\alpha}(\delta, \varepsilon, \lambda):=\delta^{2 \kappa}+\delta^{-1} \varepsilon^{2 \bar{\kappa}}+\delta \varepsilon^{-1}+\delta^{2 \alpha} \varepsilon^{-2}+\varepsilon^{-2} \lambda^{2}+\varepsilon^{-1} \lambda .
$$

Copyright (C) by SIAM. Unauthorized reproduction of this article is prohibited. 
Proposition 4.2. Let $u, \tilde{u}$ be entropy solutions of the Dirichlet problems $\mathcal{E}(A, \sigma, \xi)$, $\mathcal{E}(\tilde{A}, \tilde{\sigma}, \tilde{\xi})$, where the data satisfy Assumptions 2.1 and 2.2 , and assume that $u$ satisfies the $(\star)$-property with coefficient $\sigma$ (see Definition 4.1). Then, for every nonnegative function $\psi \in C_{c}^{\infty}(Q), \varphi \in C_{c}^{\infty}((0, T))$, and $\alpha \in\left(0, \frac{1}{2}\right)$ there exists $C \equiv C(\mathcal{S}, \alpha, \psi, \varphi)>$ 0 such that for every $\lambda, \varepsilon, \delta \in(0,1)$ we have that

$$
\begin{aligned}
& -\mathbb{E} \int_{0}^{T} \int_{Q^{2}}|u(t, x)-\tilde{u}(t, y)| \partial_{t} \varphi(t) \psi(x) \varrho_{\varepsilon}(x-y) \mathrm{d} x \mathrm{~d} y \mathrm{~d} t \\
& \leq \mathbb{E} \int_{0}^{T} \int_{Q^{2}}|A(u(t, x))-\tilde{A}(\tilde{u}(t, y))| \varphi(t) \Delta \psi(x) \varrho_{\varepsilon}(x-y) \mathrm{d} x \mathrm{~d} y \mathrm{~d} t \\
& \quad+C \varepsilon^{-2} \mathbb{E}\left(\left\|\mathbf{1}_{|u| \geq R_{\lambda}}(1+|u|)\right\|_{L_{t, x}^{m}}^{m}+\left\|\mathbf{1}_{|\tilde{u}| \geq R_{\lambda}}(1+|\tilde{u}|)\right\|_{L_{t, x}^{m}}^{m}\right) \\
& \quad+C\left(\mathcal{G}_{\alpha}(\delta, \varepsilon, \lambda)+\delta^{-1} d(\sigma, \tilde{\sigma})\right) \mathbb{E}\left(1+\|u\|_{L_{t, x}^{m+1}}^{m+1}+\|\tilde{u}\|_{L_{t, x}^{m+1}}^{m+1}\right)
\end{aligned}
$$

where $R_{\lambda}=\sup \{R \in[0, \infty]:|\mathfrak{a}(r)-\tilde{\mathfrak{a}}(r)| \leq \lambda$ for every $|r| \leq R\}$ and $\mathcal{G}_{\alpha}$ as in (4.2).

Proof. The proof is similar to [DGG19, Proof of Theorem 4.1]. The main difference lies in the presence of $\psi$ since we impose Dirichlet boundary conditions, and thus we cannot let $\psi=1$ as in the case of periodic boundary conditions dealt with in [DGG19]. We only give a sketch of the proof, highlighting the differences.

Let $\eta_{\delta}$ be a symmetric smooth approximation of $|\cdot|$ given by

$$
\eta_{\delta}(0)=\eta_{\delta}^{\prime}(0)=0, \eta_{\delta}^{\prime \prime}(r)=\delta^{-1} \tilde{\eta}\left(\delta^{-1}|r|\right)
$$

for some nonnegative $\tilde{\eta} \in C^{\infty}(\mathbb{R})$ which is bounded by 2 and supported in $(0,1)$ and integrates to 1 . Below we repeatedly use the following properties of $\eta_{\delta}$ :

$$
\left|\eta_{\delta}(r)-\right| r|| \lesssim \delta, \quad \operatorname{supp} \eta_{\delta}^{\prime \prime} \subset[-\delta, \delta], \quad \int\left|\eta_{\delta}^{\prime \prime}(r-\tilde{r})\right| \mathrm{d} \tilde{r} \leq 2, \quad\left|\eta_{\delta}^{\prime \prime}(r)\right| \leq 2 \delta^{-1} .
$$

For $y \in Q, s \in(0, T)$, and $\varepsilon, \theta>0$ sufficiently small, we also set

$\phi_{\varepsilon, \theta}(t, x, s, y):=\rho_{\theta}(t-s) \varrho_{\varepsilon}(x-y) \varphi\left(\frac{t+s}{2}\right) \psi(x), \quad \phi_{\varepsilon}(t, x, y):=\varrho_{\varepsilon}(x-y) \varphi(t) \psi(x)$.

We first apply the definition of entropy solutions with $u=u(t, x), \eta(u)=\eta_{\delta}(u-a)$, for $a \in \mathbb{R}$, and $\phi(t, x)=\phi_{\varepsilon, \theta}(t, x, s, y)$. Noting that $\phi_{\varepsilon, \theta}(0, x, s, y)=0$ for $\theta$ sufficiently small, this gives that, $\mathbb{P}$-almost surely,

$$
\begin{aligned}
-\int_{0}^{T} \int_{Q} \eta_{\delta}(u-a) \partial_{t} \phi_{\varepsilon, \theta} \mathrm{d} x \mathrm{~d} t \leq & \int_{0}^{T} \int_{Q}\left[\eta_{\delta}^{\prime}(\cdot-a) \mathfrak{a}^{2}, a\right](u) \Delta_{x} \phi_{\varepsilon, \theta} \\
& +\frac{1}{2} \phi_{\varepsilon, \theta} \eta_{\delta}^{\prime \prime}(u-a)|\sigma(x, u)|_{\ell^{2}}^{2} \mathrm{~d} x \mathrm{~d} t \\
& -\int_{0}^{T} \int_{Q} \phi_{\varepsilon, \theta} \eta_{\delta}^{\prime \prime}(u-a)\left|\nabla_{x}[\mathfrak{a}](u)\right|^{2} \mathrm{~d} x \mathrm{~d} t \\
& +\int_{0}^{T} \int_{Q} \phi_{\varepsilon, \theta} \eta_{\delta}^{\prime}(u-a) \sigma^{k}(x, u) \mathrm{d} x \mathrm{~d} \beta^{k}(t) .
\end{aligned}
$$

We now plug in $\tilde{u}(s, y)$ in place of $a$ (all the expressions are smooth functions of $a$ ) and integrate over $s, y$. Then we repeat the same procedure with the roles of $u$ and 
$\tilde{u}$ reversed, add the two resulting inequalities, and take expectations to obtain the estimate

$$
\begin{aligned}
& -\mathbb{E} \int_{t, x, s, y} \eta_{\delta}(u-\tilde{u})\left(\partial_{t} \phi_{\varepsilon, \theta}+\partial_{s} \phi_{\varepsilon, \theta}\right) \\
& \leq \leq \mathbb{E} \int_{t, x, s, y}\left(\left[\eta_{\delta}^{\prime}(\cdot-\tilde{u}) \mathfrak{a}^{2}, \tilde{u}\right](u) \Delta_{x} \phi_{\varepsilon, \theta}+\left[\eta_{\delta}^{\prime}(\cdot-u) \tilde{\mathfrak{a}}^{2}, u\right](\tilde{u}) \Delta_{y} \phi_{\varepsilon, \theta}\right) \\
& \quad-\mathbb{E} \int_{t, x, s, y} \eta_{\delta}^{\prime \prime}(u-\tilde{u})\left(\left|\nabla_{x}[\mathfrak{a}](u)\right|^{2}+\left|\nabla_{y}[\tilde{\mathfrak{a}}](\tilde{u})\right|^{2}\right) \phi_{\varepsilon, \theta} \\
& \quad+\mathbb{E} \int_{s, y}\left[\int_{t, x} \eta_{\delta}^{\prime}(u-a) \sigma^{k}(x, u) \phi_{\varepsilon, \theta} \mathrm{d} \beta^{k}(t)\right]_{a=\tilde{u}} \\
& \quad+\mathbb{E} \int_{t, x}\left[\int_{s, y} \eta_{\delta}^{\prime}(\tilde{u}-a) \tilde{\sigma}^{k}(y, \tilde{u}) \phi_{\varepsilon, \theta} \mathrm{d} \beta^{k}(s)\right]_{a=u} \\
& \quad+\mathbb{E} \int_{t, x, s, y} \frac{1}{2} \eta_{\delta}^{\prime \prime}(u-\tilde{u}) \phi_{\varepsilon, \theta}\left(|\sigma(u)|_{\ell^{2}}^{2}+|\tilde{\sigma}(\tilde{u})|_{\ell^{2}}^{2} .\right.
\end{aligned}
$$

The next step is to pass to the limit $\theta \rightarrow 0$ to obtain the estimate

$$
\begin{aligned}
-\mathbb{E} \int_{t, x, y} \eta_{\delta}(u-\tilde{u}) \partial_{t} \phi_{\varepsilon} \leq & \mathbb{E} \int_{t, x, y}\left[\eta_{\delta}^{\prime}(\cdot-\tilde{u}) \mathfrak{a}^{2}, \tilde{u}\right](u) \Delta_{x} \phi_{\varepsilon} \\
& +\left[\eta_{\delta}^{\prime}(\cdot-u) \tilde{\mathfrak{a}}^{2}, u\right](\tilde{u}) \Delta_{y} \phi_{\varepsilon} \quad\left(=: I_{1}+I_{2}\right) \\
& -\mathbb{E} \int_{t, x, y} \eta_{\delta}^{\prime \prime}(u-\tilde{u}) \phi_{\varepsilon}\left(\left|\nabla_{x}[\mathfrak{a}](u)\right|^{2}+\left|\nabla_{y}[\tilde{\mathfrak{a}}](\tilde{u})\right|^{2}\right) \quad\left(=: I_{3}\right) \\
& +\frac{1}{2} \mathbb{E} \int_{t, x, y} \eta_{\delta}^{\prime \prime}(u-\tilde{u}) \phi_{\varepsilon}|\sigma(x, u)-\tilde{\sigma}(y, \tilde{u})|_{\ell^{2}}^{2}, \quad\left(=: I_{4}\right)
\end{aligned}
$$

with $u=u(t, x)$ and $\tilde{u}=\tilde{u}(t, y)$. To do so, we first note that

$$
\partial_{t} \phi_{\varepsilon, \theta}(t, x, s, y)+\partial_{s} \phi_{\varepsilon, \theta}(t, x, s, y)=\rho_{\theta}(t-s) \varrho_{\varepsilon}(x-y)\left(\partial_{t} \varphi\right)\left(\frac{t+s}{2}\right) \psi(x) .
$$

We then use [DGG19, Proposition 3.5] to pass to the limit $\theta \rightarrow 0$ for each term in (4.3). The main difference here is the presence of $\psi$, but it is easy to see that [DGG19, Proposition 3.5] still applies in our case since $\psi$ is in $\mathcal{C}_{c}^{\infty}(Q)$. The only subtle terms in (4.3) are those involving the stochastic integrals, but they can also be treated as in [DGG19, Proof of Theorem 4.1]. For the first stochastic integral, we note that $\phi_{\varepsilon, \theta}$ vanishes for $t \notin[s, s+\theta]$, and since $\tilde{u}(s, y)$ is $\mathcal{F}_{s}$-measurable, the expectation vanishes for every $\theta>0$ by a simple factorization argument. For the second stochastic integral, we use the $(\star)$-property for $u(t, x)$ which, together with the last term in (4.3), gives $I_{4}$ in (1) if we let $\theta \rightarrow 0$. For the term $I_{1}$ we note that

$$
\begin{aligned}
I_{1}= & \mathbb{E} \int_{t, x, y}\left[\eta_{\delta}^{\prime}(\cdot-\tilde{u}) \mathfrak{a}^{2}, \tilde{u}\right](u) \Delta_{x} \phi_{\varepsilon} \\
= & -\mathbb{E} \int_{t, x, y}\left[\eta_{\delta}^{\prime}(\cdot-\tilde{u}) \mathfrak{a}^{2}, \tilde{u}\right](u) \partial_{x_{i} y_{i}}^{2} \phi_{\varepsilon} \\
& +\mathbb{E} \int_{t, x, y}\left[\eta_{\delta}^{\prime}(\cdot-\tilde{u}) \mathfrak{a}^{2}, \tilde{u}\right](u) \varphi(t) \partial_{x_{i}}\left(\partial_{x_{i}} \psi(x) \varrho_{\varepsilon}(x-y)\right) . \quad\left(=: I_{1,1}\right)
\end{aligned}
$$

Copyright $@$ by SIAM. Unauthorized reproduction of this article is prohibited. 
We furthermore note that

$$
\begin{aligned}
I_{1,1}= & -\mathbb{E} \int_{t, x, y} \int_{\tilde{u}}^{u} \int_{\tilde{u}}^{r} \eta_{\delta}^{\prime \prime}(r-\tilde{r}) \mathfrak{a}(r)^{2} \mathrm{~d} \tilde{r} \mathrm{~d} r \partial_{x_{i} y_{i}}^{2} \phi_{\varepsilon} \\
= & -\mathbb{E} \int_{\tilde{u} \leq u} \int_{\tilde{u}}^{u} \int_{\tilde{u}}^{u} \mathbf{1}_{\{\tilde{r} \leq r\}} \eta_{\delta}^{\prime \prime}(r-\tilde{r}) \mathfrak{a}(r)^{2} \mathrm{~d} \tilde{r} \mathrm{~d} r \partial_{x_{i} y_{i}}^{2} \phi_{\varepsilon} \\
& -\mathbb{E} \int_{\tilde{u} \geq u} \int_{\tilde{u}}^{u} \int_{\tilde{u}}^{u} \mathbf{1}_{\{\tilde{r} \geq r\}} \eta_{\delta}^{\prime \prime}(r-\tilde{r}) \mathfrak{a}(r)^{2} \mathrm{~d} \tilde{r} \mathrm{~d} r \partial_{x_{i} y_{i}}^{2} \phi_{\varepsilon} .
\end{aligned}
$$

Similarly we have that $I_{2}=I_{2,1}+I_{2,2}$, where

$$
\begin{aligned}
I_{2,1}:= & -\mathbb{E} \int_{\tilde{u} \leq u} \int_{\tilde{u}}^{u} \int_{\tilde{u}}^{u} \mathbf{1}_{\{\tilde{r} \leq r\}} \eta_{\delta}^{\prime \prime}(r-\tilde{r}) \tilde{\mathfrak{a}}(\tilde{r})^{2} \mathrm{~d} r \mathrm{~d} \tilde{r} \partial_{y_{i} x_{i}}^{2} \phi_{\varepsilon} \\
& -\mathbb{E} \int_{\tilde{u} \geq u} \int_{\tilde{u}}^{u} \int_{\tilde{u}}^{u} \mathbf{1}_{\{\tilde{r} \geq r\}} \eta_{\delta}^{\prime \prime}(r-\tilde{r}) \tilde{\mathfrak{a}}(\tilde{r})^{2} \mathrm{~d} r \mathrm{~d} \tilde{r} \partial_{y_{i} x_{i}}^{2} \phi_{\varepsilon}
\end{aligned}
$$

and

$$
I_{2,2}:=\mathbb{E} \int_{t, x, y}\left[\eta_{\delta}^{\prime}(\cdot-u) \tilde{\mathfrak{a}}, u\right](\tilde{u}) \varphi(t) \partial_{y_{i}}\left(\partial_{x_{i}} \psi(x) \varrho_{\varepsilon}(x-y)\right) .
$$

For $I_{3}$ as in [DGG19, Proof of Theorem 4.1], we have the bound

$$
\begin{aligned}
I_{3} \leq & 2 \mathbb{E} \int_{\tilde{u} \leq u} \int_{\tilde{u}}^{u} \int_{\tilde{u}}^{u} \mathbf{1}_{\{\tilde{r} \leq r\}} \eta_{\delta}^{\prime \prime}(r-\tilde{r}) \tilde{\mathfrak{a}}(\tilde{r}) \mathfrak{a}(r) \mathrm{d} \tilde{r} \mathrm{~d} r \partial_{x_{i} y_{i}}^{2} \phi_{\varepsilon} \\
& +2 \mathbb{E} \int_{\tilde{u} \geq u} \int_{\tilde{u}}^{u} \int_{\tilde{u}}^{u} \mathbf{1}_{\{\tilde{r} \geq r\}} \eta_{\delta}^{\prime \prime}(r-\tilde{r}) \tilde{\mathfrak{a}}(\tilde{r}) \mathfrak{a}(r) \mathrm{d} \tilde{r} \mathrm{~d} r \partial_{x_{i} y_{i}}^{2} \phi_{\varepsilon} .
\end{aligned}
$$

We now add the terms $I_{1,1}, I_{2,1}$, and $I_{3}$ to obtain the estimate

$$
I_{1,1}+I_{2,1}+I_{3} \leq \mathbb{E} \int_{t, x, y} \int_{\tilde{u}}^{u} \int_{\tilde{u}}^{u} \eta_{\delta}^{\prime \prime}(r-\tilde{r})|\mathfrak{a}(r)-\tilde{\mathfrak{a}}(\tilde{r})|^{2} \mathrm{~d} \tilde{r} \mathrm{~d} r\left|\partial_{x_{i} y_{i}}^{2} \phi_{\varepsilon}\right| .
$$

Altogether, the previous estimates imply the bound

$$
\begin{aligned}
& -\mathbb{E} \int_{t, x, y} \eta_{\delta}(u-\tilde{u}) \partial_{t} \phi_{\varepsilon} \\
& \leq \mathbb{E} \int_{t, x, y}\left[\eta_{\delta}^{\prime}(\cdot-\tilde{u}) \mathfrak{a}^{2}, \tilde{u}\right](u) \varphi(t) \partial_{x_{i}}\left(\partial_{x_{i}} \psi(x) \varrho_{\varepsilon}(x-y)\right) \quad\left(=: I_{1}^{\prime}\right) \\
& \quad+\mathbb{E} \int_{t, x, y}\left[\eta_{\delta}^{\prime}(\cdot-u) \tilde{\mathfrak{a}}^{2}, u\right](\tilde{u}) \varphi(t) \partial_{y_{i}}\left(\partial_{x_{i}} \psi(x) \varrho_{\varepsilon}(x-y)\right) \\
& \quad+\mathbb{E} \int_{t, x, y} \int_{\tilde{u}}^{u} \int_{\tilde{u}}^{u} \eta_{\delta}^{\prime \prime}(r-\tilde{r})|\mathfrak{a}(r)-\tilde{\mathfrak{a}}(\tilde{r})|^{2} \mathrm{~d} \tilde{r} \mathrm{~d} r\left|\partial_{x_{i} y_{i}}^{2} \phi_{\varepsilon}\right| \\
& \quad+\mathbb{E} \int_{t, x, y} \frac{1}{2} \eta_{\delta}^{\prime \prime}(u-\tilde{u}) \phi_{\varepsilon}|\sigma(x, u)-\tilde{\sigma}(y, \tilde{u})|_{\ell^{2}}^{2} .
\end{aligned}
$$

For the term on the left-hand side of (4.5) we have that

$$
\left|\int_{t, x, y}\left(\eta_{\delta}(u-\tilde{u})-|u-\tilde{u}|\right) \partial_{t} \phi_{\varepsilon}\right| \lesssim \delta
$$

since $\left|\eta_{\delta}(\cdot)-\right| \cdot|| \lesssim \delta$.

Copyright (c) by SIAM. Unauthorized reproduction of this article is prohibited. 
For the terms $I_{1}^{\prime}$ and $I_{2}^{\prime}$ we have that

$$
\begin{aligned}
I_{1}^{\prime}= & \mathbb{E} \int_{t, x, y}\left[\eta_{\delta}^{\prime}(\cdot-\tilde{u}) \mathfrak{a}^{2}, \tilde{u}\right](u) \varphi(t) \Delta \psi(x) \varrho_{\varepsilon}(x-y) & \left(=: I_{1,1}^{\prime}\right) \\
& +\mathbb{E} \int_{t, x, y}\left[\eta_{\delta}^{\prime}(\cdot-\tilde{u}) \mathfrak{a}^{2}, \tilde{u}\right](u) \varphi(t) \partial_{x_{i}} \psi(x) \partial_{x_{i}}\left(\varrho_{\varepsilon}(x-y)\right) & \left(=: I_{1,2}^{\prime}\right)
\end{aligned}
$$

and

$$
I_{2}^{\prime}=\mathbb{E} \int_{t, x, y}\left[\eta_{\delta}^{\prime}(\cdot-u) \tilde{\mathfrak{a}}^{2}, u\right](\tilde{u}) \varphi(t) \partial_{x_{i}} \psi(x) \partial_{y_{i}}\left(\varrho_{\varepsilon}(x-y)\right) .
$$

The term $I_{1,1}^{\prime}$ can be written as

$$
\begin{aligned}
I_{1,1}^{\prime}= & \mathbb{E} \int_{t, x, y} \int_{\tilde{u}}^{u} \operatorname{sgn}(r-\tilde{u}) \mathfrak{a}(r)^{2} \mathrm{~d} r \varphi(t) \Delta \psi(x) \varrho_{\varepsilon}(x-y) \\
& +\mathbb{E} \int_{t, x, y} \int_{\tilde{u}}^{u}\left(\eta_{\delta}^{\prime}(r-\tilde{u})-\operatorname{sgn}(r-\tilde{u})\right) \mathfrak{a}(r)^{2} \mathrm{~d} r \varphi(t) \Delta \psi(x) \varrho_{\varepsilon}(x-y) \\
= & \mathbb{E} \int_{t, x, y} \int_{\tilde{u}}^{u} \operatorname{sgn}(r-\tilde{u}) \mathfrak{a}(r)^{2} \mathrm{~d} r \varphi(t) \Delta \psi(x) \varrho_{\varepsilon}(x-y) \quad\left(=: I_{1,1,1}^{\prime}\right) \\
& +\mathbb{E} \int_{t, x, y} \int_{\tilde{u}}^{u} \mathbf{1}_{|r-\tilde{u}| \leq \delta}\left(\eta_{\delta}^{\prime}(r-\tilde{u})\right. \\
& -\operatorname{sgn}(r-\tilde{u})) \mathfrak{a}(r)^{2} \mathrm{~d} r \varphi(t) \Delta \psi(x) \varrho_{\varepsilon}(x-y) .
\end{aligned}
$$

For the term $I_{1,1,2}^{\prime}$ using the boundedness of $\Delta \psi$, the fact that $\int_{x} \varrho_{\varepsilon}(x-y) \lesssim 1$, and Assumption 2.1(a) we get that

$$
\begin{aligned}
\left|I_{1,1,2}^{\prime}\right| & \lesssim \mathbb{E} \int_{t, x, y} \int_{|r-\tilde{u}| \leq \delta} \mathfrak{a}(r)^{2} \mathrm{~d} r \varphi(t)|\Delta \psi(x)| \varrho_{\varepsilon}(x-y) \\
& \lesssim \delta \mathbb{E} \int_{t, x, y} \sup _{|r-\tilde{u}| \leq \delta} \mathfrak{a}(r)^{2} \varphi(t) \varrho_{\varepsilon}(x-y) \\
& \lesssim \delta \mathbb{E} \int_{t, y}\left(\int_{0}^{\delta+|\tilde{u}|} \mathfrak{a}^{\prime}(r) \mathrm{d} r\right)^{2} \varphi(t) \lesssim \delta \mathbb{E}\left(1+\|\tilde{u}\|_{L_{t, x}^{m}}^{m}\right) .
\end{aligned}
$$

The term $I_{1,2}^{\prime}$ can be written as

$$
\begin{aligned}
I_{1,2}^{\prime}= & \mathbb{E} \int_{t, x, y} \int_{\tilde{u}}^{u} \operatorname{sgn}(r-\tilde{u}) \mathfrak{a}(r)^{2} \mathrm{~d} r \varphi(t) \partial_{x_{i}} \psi(x) \partial_{x_{i}}\left(\varrho_{\varepsilon}(x-y)\right) \\
& +\mathbb{E} \int_{t, x, y} \int_{\tilde{u}}^{u}\left(\eta_{\delta}^{\prime}(r-\tilde{u})-\operatorname{sgn}(r-\tilde{u})\right) \mathfrak{a}(r)^{2} \mathrm{~d} r \varphi(t) \partial_{x_{i}} \psi(x) \partial_{x_{i}}\left(\varrho_{\varepsilon}(x-y)\right) \\
= & \mathbb{E} \int_{t, x, y} \int_{\tilde{u}}^{u} \operatorname{sgn}(r-\tilde{u}) \mathfrak{a}(r)^{2} \mathrm{~d} r \varphi(t) \partial_{x_{i}} \psi(x) \partial_{x_{i}}\left(\varrho_{\varepsilon}(x-y)\right) \quad\left(=: I_{1,2,1}^{\prime}\right) \\
& +\mathbb{E} \int_{t, x, y} \int_{\tilde{u}}^{u} \mathbf{1}_{|r-\tilde{u}| \leq \delta}\left(\eta_{\delta}^{\prime}(r-\tilde{u})\right. \\
& -\operatorname{sgn}(r-\tilde{u})) \mathfrak{a}(r)^{2} \mathrm{~d} r \varphi(t) \partial_{x_{i}} \psi(x) \partial_{x_{i}}\left(\varrho_{\varepsilon}(x-y)\right) .
\end{aligned}
$$

Similar calculations as in the case of $I_{1,1,2}^{\prime}$, but now using the fact that $\int_{x} \mid \partial_{x_{i}}\left(\varrho_{\varepsilon}(x-\right.$ $y)) \mid \lesssim \varepsilon^{-1}$, imply that

$$
I_{1,2,2}^{\prime} \lesssim \delta \varepsilon^{-1} \mathbb{E}\left(1+\|\tilde{u}\|_{L_{t, x}^{m}}^{m}\right)
$$

Copyright $@$ ( ) by SIAM. Unauthorized reproduction of this article is prohibited. 
Putting these estimates together gives

$$
\begin{aligned}
I_{1}^{\prime} \leq & \mathbb{E} \int_{t, x, y} \int_{\tilde{u}}^{u} \operatorname{sgn}(r-\tilde{u}) \mathfrak{a}(r)^{2} \mathrm{~d} r \varphi(t) \Delta \psi(x) \varrho_{\varepsilon}(x-y) \\
& +\mathbb{E} \int_{t, x, y} \int_{\tilde{u}}^{u} \operatorname{sgn}(r-\tilde{u}) \mathfrak{a}(r)^{2} \mathrm{~d} r \varphi(t) \partial_{x_{i}} \psi(x) \partial_{x_{i}}\left(\varrho_{\varepsilon}(x-y)\right) \\
& +C \delta \varepsilon^{-1} \mathbb{E}\left(1+\|\tilde{u}\|_{L_{t, x}^{m}}^{m}\right) .
\end{aligned}
$$

We also have

$$
\begin{array}{rlr}
I_{2}^{\prime}= & \mathbb{E} \int_{t, x, y} \int_{u}^{\tilde{u}} \operatorname{sgn}(\tilde{r}-u) \tilde{\mathfrak{a}}(\tilde{r})^{2} \mathrm{~d} \tilde{r} \varphi(t) \partial_{x_{i}} \psi(x) \partial_{y_{i}}\left(\varrho_{\varepsilon}(x-y)\right) & \left(=: I_{2,1}^{\prime}\right) \\
& +\mathbb{E} \int_{t, x, y} \int_{u}^{\tilde{u}} \mathbf{1}_{|\tilde{r}-u| \leq \delta}\left(\eta_{\delta}^{\prime}(\tilde{r}-u)\right. \\
& -\operatorname{sgn}(\tilde{r}-u)) \tilde{\mathfrak{a}}(\tilde{r})^{2} \mathrm{~d} \tilde{r} \varphi(t) \partial_{x_{i}} \psi(x) \partial_{y_{i}}\left(\varrho_{\varepsilon}(x-y)\right)
\end{array}
$$

and, as before,

$$
\begin{aligned}
I_{2}^{\prime} & \leq \mathbb{E} \int_{t, x, y} \int_{u}^{\tilde{u}} \operatorname{sgn}(\tilde{r}-u) \tilde{\mathfrak{a}}(\tilde{r})^{2} \mathrm{~d} \tilde{r} \varphi(t) \partial_{x_{i}} \psi(x) \partial_{y_{i}}\left(\varrho_{\varepsilon}(x-y)\right) \\
& +C \delta \varepsilon^{-1} \mathbb{E}\left(1+\|u\|_{L_{t, x}^{m}}^{m}\right)
\end{aligned}
$$

The term $I_{3}^{\prime}$ can be treated exactly as in [DGG19, Proof of Theorem 4.1] to obtain

$$
\begin{aligned}
I_{3}^{\prime} \lesssim & \varepsilon^{-2}\left(\delta^{2 \alpha}+\lambda^{2}\right) \mathbb{E}\left(1+\|u\|_{L_{t, x}^{m}}^{m}+\|\tilde{u}\|_{L_{t, x}^{m}}^{m}\right) \\
& +\varepsilon^{-2}\left(\mathbb{E}\left\|\mathbf{1}_{|u| \geq R_{\lambda}}(1+|u|)\right\|_{L_{t, x}^{m}}^{m}+\mathbb{E}\left\|\mathbf{1}_{|\tilde{u}| \geq R_{\lambda}}(1+|\tilde{u}|)\right\|_{L_{t, x}^{m}}^{m}\right) .
\end{aligned}
$$

For the term $I_{4}^{\prime}$ we notice that

$$
\begin{aligned}
I_{4}^{\prime} \lesssim & \mathbb{E} \int_{t, x, y} \eta_{\delta}^{\prime \prime}(u-\tilde{u}) \phi_{\varepsilon}\left(|\sigma(x, u)-\sigma(x, \tilde{u})|_{\ell^{2}}^{2}+\mid \sigma(x, u)\right. \\
& \left.-\left.\sigma(y, \tilde{u})\right|_{\ell^{2}} ^{2}+|\sigma(y, \tilde{u})-\tilde{\sigma}(y, \tilde{u})|_{\ell^{2}}^{2}\right) \\
\lesssim & \left(\delta^{2 \kappa}+\delta^{-1} \varepsilon^{2 \bar{\kappa}}+\delta^{-1} d(\sigma, \tilde{\sigma})\right) \mathbb{E}\left(1+\|u\|_{L_{t, x}^{m+1}}^{m+1}+\|\tilde{u}\|_{L_{t, x}^{m+1}}^{m+1}\right),
\end{aligned}
$$

where we use Assumption 2.2 and the fact that $\left|\eta_{\delta}^{\prime \prime}\right| \lesssim \delta^{-1}$.

Noting that $\partial_{y_{i}}\left(\varrho_{\varepsilon}(x-y)\right)=-\partial_{x_{i}}\left(\varrho_{\varepsilon}(x-y)\right)$ and that for every $u, \tilde{u}$, and nondecreasing differentiable function $A$, we have the identity

$$
\int_{\tilde{u}}^{u} \operatorname{sgn}(r-\tilde{u}) A^{\prime}(r) d r=|A(u)-A(\tilde{u})|
$$

Copyright $@$ by SIAM. Unauthorized reproduction of this article is prohibited. 
we obtain by (4.6)-(4.9) (and the fact that $\delta \leq \delta^{2 \kappa}$ )

$$
\begin{aligned}
- & \mathbb{E} \int_{0}^{T} \int_{Q^{2}}|u-\tilde{u}| \partial_{t} \varphi(t) \psi(x) \varrho_{\varepsilon}(x-y) \mathrm{d} x \mathrm{~d} y \mathrm{~d} t \\
\leq & \mathbb{E} \int_{0}^{T} \int_{Q^{2}}|A(u)-A(\tilde{u})| \varphi(t) \Delta \psi(x) \varrho_{\varepsilon}(x-y) \mathrm{d} x \mathrm{~d} y \mathrm{~d} t \\
& +\mathbb{E} \int_{0}^{T} \int_{Q^{2}}|A(u)-A(\tilde{u})| \varphi(t) \partial_{x_{i}} \psi(x) \partial_{x_{i}}\left(\varrho_{\varepsilon}(x-y)\right) \mathrm{d} x \mathrm{~d} y \mathrm{~d} t \\
& -\mathbb{E} \int_{0}^{T} \int_{Q^{2}}|\tilde{A}(u)-\tilde{A}(\tilde{u})| \varphi(t) \partial_{x_{i}} \psi(x) \partial_{x_{i}}\left(\varrho_{\varepsilon}(x-y)\right) \mathrm{d} x \mathrm{~d} y \mathrm{~d} t \\
& +C \varepsilon^{-2} \mathbb{E}\left(\left\|\mathbf{1}_{|u| \geq R_{\lambda}}(1+|u|)\right\|_{L_{t, x}^{m}}^{m}+\left\|\mathbf{1}_{|\tilde{u}| \geq R_{\lambda}}(1+|\tilde{u}|)\right\|_{L_{t, x}^{m}}^{m}\right) \\
& +C\left(\mathcal{G}_{\alpha}(\delta, \varepsilon, \lambda)+\delta^{-1} d(\sigma, \tilde{\sigma})\right) \mathbb{E}\left(1+\|u\|_{L_{t, x}^{m+1}}^{m+1}+\|\tilde{u}\|_{L_{t, x}^{m+1}}^{m+1}\right) .
\end{aligned}
$$

By the triangle inequality, we have that

$$
\begin{aligned}
& \mathbb{E} \int_{0}^{T} \int_{Q^{2}}(|A(u)-A(\tilde{u})|-|\tilde{A}(u)-\tilde{A}(\tilde{u})|) \varphi(t) \partial_{x_{i}} \psi(x) \partial_{x_{i}}\left(\varrho_{\varepsilon}(x-y)\right) \mathrm{d} x \mathrm{~d} y \mathrm{~d} t \\
& \leq \mathbb{E} \int_{0}^{T} \int_{Q^{2}}(|A(u)-\tilde{A}(u)|+|A(\tilde{u})-\tilde{A}(\tilde{u})|) \varphi(t)\left|\partial_{x_{i}} \psi(x) \partial_{x_{i}}\left(\varrho_{\varepsilon}(x-y)\right)\right| \mathrm{d} x \mathrm{~d} y \mathrm{~d} t \\
& \lesssim \varepsilon^{-1} \lambda \mathbb{E}\left(\|u\|_{L_{t, x}^{\frac{m+1}{2}}}^{\frac{m+1}{2}}+\|\tilde{u}\|_{L_{t, x}^{\frac{m+1}{2}}}^{\frac{m+1}{2}}\right)+\varepsilon^{-1} \mathbb{E}\left(\left\|\mathbf{1}_{|u| \geq R_{\lambda}} u\right\|_{L_{t, x}^{m}}^{m}+\left\|\mathbf{1}_{|\tilde{u}| \geq R_{\lambda}} \tilde{u}\right\|_{L_{t, x}^{m}}^{m}\right),
\end{aligned}
$$

where in the last step we use the facts that $\int_{y}\left|\partial_{x_{i}}\left(\varrho_{\varepsilon}(x-y)\right)\right|, \int_{x}\left|\partial_{x_{i}}\left(\varrho_{\varepsilon}(x-y)\right)\right| \lesssim \varepsilon^{-1}$ and that for every $r \in \mathbb{R}$, by Assumption 2.1(a),

$$
\begin{aligned}
|A(r)-\tilde{A}(r)| & \leq \int_{0}^{|r|}\left|\mathfrak{a}(\zeta)^{2}-\tilde{\mathfrak{a}}(\zeta)^{2}\right| \mathrm{d} \zeta \\
& \lesssim \lambda \int_{0}^{|r|}(|\mathfrak{a}(\zeta)|+|\tilde{\mathfrak{a}}(\zeta)|) \mathrm{d} \zeta+\mathbf{1}_{|r| \geq R_{\lambda}} \int_{0}^{|r|}\left(\mathfrak{a}(\zeta)^{2}+\tilde{\mathfrak{a}}(\zeta)^{2}\right) \mathrm{d} \zeta \\
& \lesssim \lambda|r|^{\frac{m+1}{2}}+\mathbf{1}_{|r| \geq R_{\lambda}}|r|^{m} .
\end{aligned}
$$

Similarly, since $\int_{x}\left|\varrho_{\varepsilon}(x-y)\right| \lesssim 1$, we see that

$$
\begin{aligned}
& \mathbb{E} \int_{0}^{T} \int_{Q^{2}}(|A(u)-A(\tilde{u})|-|A(u)-\tilde{A}(\tilde{u})|) \varphi(t) \Delta \psi(x) \varrho_{\varepsilon}(x-y) \mathrm{d} x \mathrm{~d} y \mathrm{~d} t \\
& \lesssim \lambda \mathbb{E}\left(\|\tilde{u}\|_{L_{t, x}^{\frac{m+1}{2}}}^{\frac{m+1}{2}}\right)+\mathbb{E}\left(\left\|\mathbf{1}_{|\tilde{u}| \geq R_{\lambda}} \tilde{u}\right\|_{L_{t, x}^{m}}^{m}\right) .
\end{aligned}
$$

By (4.10)-(4.12) we obtain the desired inequality.

The next lemma is a pointwise in time version of Proposition 4.2.

Lemma 4.3. Let $u, \tilde{u}$ be entropy solutions of the Dirichlet problems $\mathcal{E}(A, \sigma, \xi)$, $\mathcal{E}(\tilde{A}, \tilde{\sigma}, \tilde{\xi})$, where the data satisfy Assumptions 2.1 and 2.2 , and assume that $u$ satisfies

Copyright (c) by SIAM. Unauthorized reproduction of this article is prohibited. 
the $(\star)$-property with coefficient $\sigma$ (see Definition 4.1). Then, for every $\psi \in C_{c}^{\infty}(Q)$ and $\alpha \in\left(0, \frac{1}{2}\right)$ there exists $C \equiv C(\mathcal{S}, \alpha, \psi)>0$ such that for every $\lambda, \varepsilon, \delta \in(0,1)$ and every right Lebesgue point $s \leq t$ of the mapping

$$
\tau \mapsto \mathbb{E} \int_{x, y}|u(\tau, x)-\tilde{u}(\tau, y)| \psi(x) \varrho_{\varepsilon}(x-y)
$$

we have that

$$
\begin{aligned}
& \mathbb{E} \int_{Q^{2}}|u(t, x)-\tilde{u}(t, y)| \psi(x) \varrho_{\varepsilon}(x-y) \mathrm{d} x \mathrm{~d} y \\
& \leq \mathbb{E} \int_{Q^{2}}|u(s, x)-\tilde{u}(s, y)| \psi(x) \varrho_{\varepsilon}(x-y) \mathrm{d} x \mathrm{~d} y \\
& \quad+\mathbb{E} \int_{s}^{t} \int_{Q^{2}}|A(u(\tau, x))-\tilde{A}(\tilde{u}(\tau, y))| \Delta \psi(x) \varrho_{\varepsilon}(x-y) \mathrm{d} x \mathrm{~d} y \mathrm{~d} \tau \\
& \quad+C \varepsilon^{-2} \mathbb{E}\left(\left\|\mathbf{1}_{|u| \geq R_{\lambda}}(1+|u|)\right\|_{L_{t, x}^{m}}^{m}+\left\|\mathbf{1}_{|\tilde{u}| \geq R_{\lambda}}(1+|\tilde{u}|)\right\|_{L_{t, x}^{m}}^{m}\right) \\
& \quad+C\left(\mathcal{G}_{\alpha}(\delta, \varepsilon, \lambda)+\delta^{-1} d(\sigma, \tilde{\sigma})\right) \mathbb{E}\left(1+\|u\|_{L_{t, x}^{m+1}}^{m+1}+\|\tilde{u}\|_{L_{t, x}^{m+1}}^{m+1}\right),
\end{aligned}
$$

where $R_{\lambda}=\sup \{R \in[0, \infty]:|\mathfrak{a}(r)-\tilde{\mathfrak{a}}(r)| \leq \lambda$ for every $|r| \leq R\}$ and $\mathcal{G}_{\alpha}$ as in (4.2).

Proof. The proof is given in [DGG19, Proof of Theorem 4.1] by approximating the function $\mathbf{1}_{[s, t]}$ and using Proposition 4.2.

We are now ready to prove the main result of this section.

Lemma 4.4. Let $u, \tilde{u}$ be entropy solutions of the Dirichlet problems $\mathcal{E}(A, \sigma, \xi)$, $\mathcal{E}(\tilde{A}, \tilde{\sigma}, \tilde{\xi})$, where the data satisfy Assumptions 2.1 and 2.2 , and assume that $u$ satisfies the $(\star)$-property with coefficient $\sigma$ (see Definition 4.1).

1. For every $\psi \in C_{c}^{\infty}(Q)$ and $\alpha \in\left(0, \frac{1}{2}\right)$ there exists $C \equiv C(\mathcal{S}, \alpha, \psi)>0$ such that for every $\lambda, \delta \in(0,1)$ and $\varepsilon \in(0,1)$ sufficiently small,

$$
\begin{aligned}
\mathbb{E} \int_{0}^{T} & \int_{Q}|u(t, x)-\tilde{u}(t, x)| \psi(x) \mathrm{d} x \mathrm{~d} t \\
\leq & T \mathbb{E} \int_{Q}|\xi(x)-\tilde{\xi}(x)| \psi(x) \mathrm{d} x+T \sup _{|h| \leq \varepsilon} \mathbb{E} \int_{Q}|\tilde{\xi}(x)-\tilde{\xi}(x+h)| \psi(x) \mathrm{d} x \\
& +\mathbb{E} \int_{0}^{T} \int_{0}^{t} \int_{Q}|A(u(\tau, x))-\tilde{A}(\tilde{u}(\tau, x))| \Delta \psi(x) \mathrm{d} x \mathrm{~d} \tau \mathrm{d} t \\
& +C \varepsilon^{\frac{2}{m+1}} \mathbb{E}\left(1+\|\nabla[\tilde{\mathfrak{a}}](\tilde{u})\|_{L_{t, x}^{1}}\right) \\
& +C \varepsilon \mathbb{E}\|\nabla \tilde{A}(\tilde{u})\|_{L_{t, x}^{1}}+C \varepsilon^{-2} \mathbb{E}\left(\left\|\mathbf{1}_{|u| \geq R_{\lambda}}(1+|u|)\right\|_{L_{t, x}^{m}}^{m}\right. \\
& \left.+\left\|\mathbf{1}_{|\tilde{u}| \geq R_{\lambda}}(1+|\tilde{u}|)\right\|_{L_{t, x}^{m}}^{m}\right) \\
& +C\left(\mathcal{G}_{\alpha}(\delta, \varepsilon, \lambda)+\delta^{-1} d(\sigma, \tilde{\sigma})\right) \mathbb{E}\left(1+\|u\|_{L_{t, x}^{m+1}}^{m+1}+\|\tilde{u}\|_{L_{t, x}^{m+1}}^{m+1}\right)
\end{aligned}
$$

where $R_{\lambda}=\sup \{R \in[0, \infty]:|\mathfrak{a}(r)-\tilde{\mathfrak{a}}(r)| \leq \lambda$ for every $|r| \leq R\}$ and $\mathcal{G}_{\alpha}$ as in (4.2).

Copyright $@$ ( ) by SIAM. Unauthorized reproduction of this article is prohibited. 
2. If we furthermore assume that $\tilde{A}=A$ and $\tilde{\sigma}=\sigma$, then for every $\psi \in C_{c}^{\infty}(Q)$ and almost every $s<t \leq T$ we have that

$$
\begin{aligned}
& \mathbb{E} \int_{Q}|u(t, x)-\tilde{u}(t, x)| \psi(x) \mathrm{d} x-\mathbb{E} \int_{Q}|u(s, x)-\tilde{u}(s, x)| \psi(x) \mathrm{d} x \\
& \quad \leq \mathbb{E} \int_{s}^{t} \int_{Q}|A(u(\tau, x))-A(\tilde{u}(\tau, x))| \Delta \psi(x) \mathrm{d} x \mathrm{~d} \tau .
\end{aligned}
$$

In addition, this estimate holds for $s=0$ and $u(s), \tilde{u}(s)$ replaced by $\xi, \tilde{\xi}$.

Proof of Lemma 4.4-1. We first notice that for all $\varepsilon \in(0,1)$ sufficiently small, by the mean value theorem for $\tilde{A}(\tilde{u}(\tau, \cdot))$ we have that

$$
\begin{aligned}
& \mathbb{E} \int_{s}^{t} \int_{Q^{2}}|A(u(\tau, x))-\tilde{A}(\tilde{u}(\tau, y))| \Delta \psi(x) \varrho_{\varepsilon}(x-y) \mathrm{d} x \mathrm{~d} y \mathrm{~d} \tau \\
& \quad \leq \mathbb{E} \int_{s}^{t} \int_{Q}|A(u(\tau, x))-\tilde{A}(\tilde{u}(\tau, x))| \Delta \psi(x) \mathrm{d} x \mathrm{~d} \tau+C \varepsilon \mathbb{E}\|\nabla \tilde{A}(\tilde{u})\|_{L_{t, x}^{1}}
\end{aligned}
$$

for some $C \equiv C\left(\mathcal{S},\|\Delta \psi\|_{L_{x}^{\infty}}\right)>0$, where we also use the fact that $\int_{y}\left|(x-y) \varrho_{\varepsilon}(x-y)\right| \lesssim \varepsilon$. Then, by Lemma 4.3 we have that for every right Lebesgue point $s \leq t$ of the mapping (4.13),

$$
\begin{aligned}
& \mathbb{E} \int_{Q^{2}}|u(t, x)-\tilde{u}(t, y)| \psi(x) \varrho_{\varepsilon}(x-y) \mathrm{d} x \mathrm{~d} y \\
& \leq \mathbb{E} \int_{Q^{2}}|u(s, x)-\tilde{u}(s, y)| \psi(x) \varrho_{\varepsilon}(x-y) \mathrm{d} x \mathrm{~d} y \\
& \quad+\mathbb{E} \int_{s}^{t} \int_{Q}|A(u(\tau, x))-\tilde{A}(\tilde{u}(\tau, x))| \Delta \psi(x) \mathrm{d} x \mathrm{~d} \tau+M,
\end{aligned}
$$

where $M \equiv M(\delta, \varepsilon, \lambda)>0$ is given by

$$
\begin{aligned}
M:= & C \varepsilon^{-2} \mathbb{E}\left(\left\|\mathbf{1}_{|u| \geq R_{\lambda}}(1+|u|)\right\|_{L_{t, x}^{m}}^{m}+\left\|\mathbf{1}_{|\tilde{u}| \geq R_{\lambda}}(1+|\tilde{u}|)\right\|_{L_{t, x}^{m}}^{m}\right) \\
& +C\left(\mathcal{G}_{\alpha}(\delta, \varepsilon, \lambda)+\delta^{-1} d(\sigma, \tilde{\sigma})\right) \mathbb{E}\left(1+\|u\|_{L_{t, x}^{m+1}}^{m+1}+\|\tilde{u}\|_{L_{t, x}^{m+1}}^{m+1}\right)+C \varepsilon \mathbb{E}\|\nabla \tilde{A}(\tilde{u})\|_{L_{t, x}^{1}} .
\end{aligned}
$$

As in [DGG19, Lemma 3.2] we can prove that

$$
\lim _{h \rightarrow 0} \frac{1}{h} \int_{0}^{h} \mathbb{E} \int_{Q}|u(s, x)-\xi(x)|^{2} \psi(x)^{2} \mathrm{~d} x \mathrm{~d} s=0,
$$

and similarly for $\tilde{u}, \tilde{\xi}$. Hence, if we integrate (4.16) over $s \in(0, h)$, divide by $h$, and let $h \rightarrow 0$, we get

$$
\begin{aligned}
& \mathbb{E} \int_{Q^{2}}|u(t, x)-\tilde{u}(t, y)| \psi(x) \varrho_{\varepsilon}(x-y) \mathrm{d} x \mathrm{~d} y \leq \mathbb{E} \int_{Q^{2}}|\xi(x)-\tilde{\xi}(y)| \psi(x) \varrho_{\varepsilon}(x-y) \mathrm{d} x \mathrm{~d} y \\
& +\mathbb{E} \int_{0}^{t} \int_{Q}|A(u(\tau, x))-\tilde{A}(\tilde{u}(\tau, x))| \Delta \psi(x) \mathrm{d} x \mathrm{~d} \tau+M .
\end{aligned}
$$

Copyright $@$ ㅇ by SIAM. Unauthorized reproduction of this article is prohibited. 
We now integrate the above inequality over $t \in[0, T]$ to obtain

$$
\begin{aligned}
& \mathbb{E} \int_{0}^{T} \int_{Q^{2}}|u(t, x)-\tilde{u}(t, y)| \psi(x) \varrho_{\varepsilon}(x-y) \mathrm{d} x \mathrm{~d} y \mathrm{~d} t \\
& \leq T \mathbb{E} \int_{Q^{2}}|\xi(x)-\tilde{\xi}(y)| \psi(x) \varrho_{\varepsilon}(x-y) \mathrm{d} x \mathrm{~d} y \\
& \quad \mathbb{E} \int_{0}^{T} \int_{0}^{t} \int_{Q}|A(u(\tau, x))-\tilde{A}(\tilde{u}(\tau, x))| \Delta \psi(x) \mathrm{d} x \mathrm{~d} \tau \mathrm{d} t+T M .
\end{aligned}
$$

Moreover, for every $\varepsilon \in(0,1)$ sufficiently small such that $x+h \in Q$ whenever $x \in$ $\operatorname{supp} \psi$ and $|h| \leq \varepsilon$, we have that

$$
\begin{aligned}
\mathbb{E} \int_{Q^{2}}|\xi(x)-\tilde{\xi}(y)| \psi(x) \varrho_{\varepsilon}(x-y) \mathrm{d} x \mathrm{~d} y \leq & \sup _{|h| \leq \varepsilon} \mathbb{E} \int_{Q}|\tilde{\xi}(x)-\tilde{\xi}(x+h)| \psi(x) \mathrm{d} x \\
& +\mathbb{E} \int_{Q}|\xi(x)-\tilde{\xi}(x)| \psi(x) \mathrm{d} x
\end{aligned}
$$

We finally note that by [DGG19, Lemma 3.1],

$$
\begin{aligned}
& \mathbb{E}\left|\int_{0}^{T} \int_{Q^{2}}\right| u(t, x)-\tilde{u}(t, y)\left|\psi(x) \varrho_{\varepsilon}(x-y) \mathrm{d} x \mathrm{~d} y \mathrm{~d} t-\int_{0}^{T} \int_{Q}\right| u(t, x)-\tilde{u}(t, x)|\psi(x) \mathrm{d} x \mathrm{~d} t| \\
& \quad \lesssim \varepsilon^{\frac{2}{m+1}}\left(1+\mathbb{E}\|\nabla[\tilde{\mathfrak{a}}](\tilde{u})\|_{L_{t, x}^{1}}\right) .
\end{aligned}
$$

This implies the assertion.

Proof of Lemma 4.4-2. We first notice that since $\tilde{A}=A$, we can choose $\lambda=0$ and $R_{\lambda}=\infty$ in (4.14). Since we also have that $d(\sigma, \tilde{\sigma})=0$, using again (4.15), (4.14) reads as follows:

$$
\begin{aligned}
& \mathbb{E} \int_{Q^{2}}|u(t, x)-\tilde{u}(t, y)| \psi(x) \varrho_{\varepsilon}(x-y) \mathrm{d} x \mathrm{~d} y \\
& \leq \mathbb{E} \int_{Q^{2}}|u(s, x)-\tilde{u}(s, y)| \psi(x) \varrho_{\varepsilon}(x-y) \mathrm{d} x \mathrm{~d} y \\
& \quad+\mathbb{E} \int_{s}^{t} \int_{Q}|A(u(\tau, x))-A(\tilde{u}(\tau, x))| \Delta \psi(x) \mathrm{d} x \mathrm{~d} \tau \\
& \quad+C \mathcal{G}_{\alpha}(\delta, \varepsilon, 0) \mathbb{E}\left(1+\|u\|_{L_{t, x}^{m+1}}^{m+1}+\|\tilde{u}\|_{L_{t, x}^{m+1}}^{m+1}\right) \\
& \quad+C \varepsilon \mathbb{E}\|\nabla \tilde{A}(\tilde{u})\|_{L_{t, x}^{1}}
\end{aligned}
$$

for almost every $s \leq t \leq T$. Note that by (4.17), $\tau=0$ is a right Lebesgue point of (4.13), and hence the last inequality holds also for $s=0$. As in [DGG19, Proof of Theorem 4.1], we pass to the limit $\varepsilon, \delta \rightarrow 0$ simultaneously by choosing $\delta$ depending on $\varepsilon$. More specifically, we choose $\nu \in\left((m \wedge 2)^{-1}, \bar{\kappa}\right)$ and $\alpha<1 \wedge \frac{m}{2}$ such that $(2 \alpha)(2 \nu)>2$ and set $\delta=\varepsilon^{2 \nu}$. Letting $\varepsilon \rightarrow 0$ proves the desired estimate.

In the next corollary we replace $\psi$ in Lemma $4.4-2$ by $w$ as in (1.7) which implies an estimate in $L_{w ; x}^{1}$.

Copyright $@$ by SIAM. Unauthorized reproduction of this article is prohibited. 
COROLLARY 4.5. Under the assumptions of Lemma 4.4-2 we have that for almost every $s<t \leq T$,

$$
\mathbb{E}\|u(t)-\tilde{u}(t)\|_{L_{w ; x}^{1}} \leq \mathbb{E}\|u(s)-\tilde{u}(s)\|_{L_{w ; x}^{1}}-\mathbb{E} \int_{s}^{t}\|A(u(\tau))-A(\tilde{u}(\tau))\|_{L_{x}^{1}} \mathrm{~d} \tau .
$$

In addition, the following estimate holds:

$$
\sup _{t \in[0, T]} \operatorname{ess} \mathbb{E}\|u(t)-\tilde{u}(t)\|_{L_{w ; x}^{1}} \leq \mathbb{E}\|\xi-\tilde{\xi}\|_{L_{w ; x}^{1}} .
$$

Proof. We choose a sequence of nonnegative functions $\psi_{n} \in C_{c}^{\infty}(Q)$ such that $\psi_{n} \rightarrow w$ in $H_{0, x}^{1}$. Using the facts that $|A(u)-A(\tilde{u})| \in L_{\omega, t}^{2} H_{0, x}^{1}$ and that $w$ solves (1.7), by virtue of Lemma 4.4-2 we get that for almost every $s<t \leq T$ (including $s=0)$,

$$
\begin{aligned}
& \mathbb{E} \int_{Q}|u(t, x)-\tilde{u}(t, x)| w(x) \mathrm{d} x \\
& \leq \int_{Q}|u(s, x)-\tilde{u}(s, x)| w(x) \mathrm{d} x-\mathbb{E} \int_{s}^{t} \int_{Q}|A(u(\tau, x))-A(\tilde{u}(\tau, x))| \mathrm{d} x \mathrm{~d} \tau,
\end{aligned}
$$

which proves the desired estimate.

\section{Proofs of well-posedness.}

5.1. Proofs of Theorems 3.1 and 3.2. In this section we first prove Theorem 3.1 on the existence and uniqueness of solutions to $\mathcal{E}(A, \sigma, \xi)$. From Corollary 4.5 (see (4.19)) it follows that each pair of entropy solutions of $\mathcal{E}(A, \sigma, \xi)$ coincides, provided that one of them satisfies the $(\star)$-property (see Definition 4.1 ). Hence, in order to conclude the existence and uniqueness of entropy solutions, it suffices to show the existence of an entropy solution satisfying the $(\star)$-property. To do so, we use a vanishing viscosity approximation. The (probabilistically) strong existence of solutions for the approximating equations is quite standard by now. It relies on a technique from [GK96], where a characterization of the convergence in probability is used to show that weak existence combined with strong uniqueness implies strong existence. This has been used in the past in the context of SPDEs (see [Hof13, GH18] and the references therein). Proofs are included in Appendix A for the convenience of the reader.

For the proof of the following proposition we refer the reader to [DGG19, Proposition 5.1].

Proposition 5.1. Let A satisfy Assumption 2.1(a) with a constant $K \geq 1$. Then, for every $n \geq 1$ there exists an increasing function $A_{n} \in C^{\infty}(\mathbb{R})$ with bounded derivatives, satisfying Assumption 2.1 (a) with constant $3 K$, such that $\mathfrak{a}_{n}(r) \geq \frac{2}{n}$, and

$$
\sup _{|r| \leq n}\left|\mathfrak{a}(r)-\mathfrak{a}_{n}(r)\right| \leq \frac{4}{n}
$$

Let $A_{n}$ be as above, and set

$$
\xi_{n}:=(-n) \vee(\xi \wedge n), \quad \sigma_{n}:=\rho_{\frac{1}{n}}^{\otimes(d+1)} * \sigma(\cdot,-n \vee(\cdot \wedge n))
$$

Copyright $@$ by SIAM. Unauthorized reproduction of this article is prohibited. 
Definition 5.2. An $L_{x}^{2}$-solution of the Dirichlet problem $\mathcal{E}\left(A_{n}, \sigma_{n}, \xi_{n}\right)$ is a continuous $L_{x}^{2}$-valued process $u_{n}$, such that $u_{n}, A_{n}\left(u_{n}\right) \in L_{\omega, t}^{2} H_{0 ; x}^{1}$, and the equality

$$
\left(u_{n}(t, \cdot), \phi\right)=\left(\xi_{n}, \phi\right)-\int_{0}^{t}\left(\nabla A_{n}\left(u_{n}(s, \cdot)\right), \nabla \phi\right) d s+\int_{0}^{t}\left(\sigma_{n}^{k}\left(\cdot, u_{n}(s, \cdot)\right), \phi\right) \mathrm{d} \beta^{k}(s)
$$

holds for every $\phi \in C_{c}^{\infty}(Q), \mathbb{P}$-almost surely, for every $t \in[0, T]$.

If $u_{n}$ is an $L_{x}^{2}$-solution of $\mathcal{E}\left(A_{n}, \sigma_{n}, \xi_{n}\right)$, then by standard arguments (see also [DGG19, p. 24]) one obtains

$$
\begin{gathered}
\mathbb{E} \sup _{t \leq T}\left\|u_{n}\right\|_{L_{x}^{2}}^{p}+\mathbb{E}\left\|\nabla\left[\mathfrak{a}_{n}\right]\left(u_{n}\right)\right\|_{L_{t, x}^{2}}^{p} \lesssim p 1+\mathbb{E}\left\|\xi_{n}\right\|_{L_{x}^{2}}^{p}, \\
\mathbb{E} \sup _{t \leq T}\left\|u_{n}\right\|_{L_{x}^{m+1}}^{m+1}+\mathbb{E}\left\|\nabla A_{n}\left(u_{n}\right)\right\|_{L_{t, x}^{2}}^{2} \lesssim 1+\mathbb{E}\left\|\xi_{n}\right\|_{L_{x}^{m+1}}^{m+1},
\end{gathered}
$$

where the implicit constants do not depend on $n$. Notice that $\left|\xi_{n}\right|$ is bounded by $n$, which implies that the right-hand side of the last two inequalities is finite. Moreover, by the construction of $\xi_{n}$ one concludes that for every $p \geq 2$,

$$
\mathbb{E} \sup _{t \leq T}\left\|u_{n}\right\|_{L_{x}^{2}}^{p}+\mathbb{E}\left\|\nabla\left[\mathfrak{a}_{n}\right]\left(u_{n}\right)\right\|_{L_{t, x}^{2}}^{p} \lesssim_{p} 1+\mathbb{E}\|\xi\|_{L_{x}^{2}}^{p}
$$

and

$$
\mathbb{E} \sup _{t \leq T}\left\|u_{n}\right\|_{L_{x}^{m+1}}^{m+1}+\mathbb{E}\left\|\nabla A_{n}\left(u_{n}\right)\right\|_{L_{t, x}^{2}}^{2} \lesssim 1+\mathbb{E}\|\xi\|_{L_{x}^{m+1}}^{m+1} .
$$

Finally, since $\mathfrak{a}_{n} \geq \frac{2}{n}>0$, we have $\left|\nabla u_{n}\right| \leq C_{n}\left|\nabla\left[\mathfrak{a}_{n}\right]\left(u_{n}\right)\right|$, and so by (5.5), we have the ( $n$-dependent) bound

$$
\mathbb{E}\left\|\nabla u_{n}\right\|_{L_{t, x}^{2}}^{p}<\infty
$$

The proofs of the next two lemmas are the same as those of [DGG19, Lemma 5.2] and [DGG19, Corollary 3.4] and are therefore omitted.

Lemma 5.3. For $n \geq 1$, let $u_{n}$ be an $L_{x}^{2}$-solution of $\mathcal{E}\left(A_{n}, \sigma_{n}, \xi_{n}\right)$. Then, $u_{n}$ satisfies the ( $\star$ )-property (4.1) with coefficient $\sigma_{n}$ and $C \equiv C(\mathcal{S}, n)>0$. If, in addition, $\|\xi\|_{L_{x}^{2}}$ has moments of order 4 , then the constant $C$ is independent of $n$.

Lemma 5.4. Let $u_{n}$ be a sequence bounded in $L_{\omega, t}^{m+1} L_{x}^{m+1}$, satisfying the ( $\star$ )property (4.1) with coefficient $\sigma_{n}$, uniformly in $n$. Suppose that $u_{n}$ converges for almost every $(\omega, t, x)$ to a function $u$ and that $\lim _{n \rightarrow \infty} d\left(\sigma_{n}, \sigma\right)=0$. Then $u$ has the $(\star)$-property with coefficient $\sigma$.

The proof of the next proposition is given in Appendix A.

Proposition 5.5. Suppose Assumptions 2.1 and 2.2 hold. Then, for every $n \geq 1$, $\mathcal{E}\left(A_{n}, \sigma_{n}, \xi_{n}\right)$ has a unique $L_{x}^{2}$-solution $u_{n}$.

We are now ready to prove Theorem 3.1.

Proof of Theorem 3.1. Step 1. We first assume that $\mathbb{E}\|\xi\|_{L_{x}^{2}}^{4}<\infty$. For $n \geq 1$, let $u_{n}$ be the unique $L_{x}^{2}$-solution of $\mathcal{E}\left(A_{n}, \sigma_{n}, \xi_{n}\right)$ by Proposition 5.5. We will show that $\left(u_{n}\right)_{n \geq 1}$ is a Cauchy sequence in $L_{\omega, t}^{1} L_{w ; x}^{1}$. Let $N \geq 1$ be arbitrary. As in the conclusion of the proof of Lemma 4.4-2, we choose $\nu$ such that $\nu \in\left((m \wedge 2)^{-1}, \bar{\kappa}\right)$, and then we choose $\alpha<1 \wedge \frac{m}{2}$ such that $-2+(2 \alpha)(2 \nu)>0$, so that $\mathcal{G}_{\alpha}\left(\varepsilon^{2 \nu}, \varepsilon, 0\right) \rightarrow 0$ 
as $\varepsilon \rightarrow 0$. Then, let $\psi_{l} \in C_{c}^{\infty}(Q)$ be a sequence of nonnegative functions such that $\left\|\psi_{l}-w\right\|_{H_{0 ; x}^{1}} \rightarrow 0$ as $l \rightarrow \infty$. We apply Lemma $4.4-1$ to $u_{n}$ and $u_{n^{\prime}}$, for arbitrary $n \leq n^{\prime}$ setting $\delta=\varepsilon^{2 \nu}$, and $\lambda=\frac{8}{n}$. By (5.1) we have that $R_{\lambda} \geq n$ (see the statement of Lemma 4.4-1 for the definition of $R_{\lambda}$ ). Recalling the uniform estimates (5.5)-(5.6) and the triangle inequality

$$
\mathbb{E}\left\|\xi_{n^{\prime}}(\cdot)-\xi_{n^{\prime}}(\cdot+h)\right\|_{L_{x}^{1}} \leq \mathbb{E}\|\xi(\cdot)-\xi(\cdot+h)\|_{L_{x}^{1}}+2 \mathbb{E}\left\|\xi-\xi_{n^{\prime}}\right\|_{L_{x}^{1}},
$$

where for convenience we have extended $\xi_{n}$ and $\xi$ on $\mathbb{R}^{d}$ by setting them equal to 0 in $Q^{c}$, we have

$$
\begin{aligned}
\mathbb{E}\left\|\left(u_{n}-u_{n^{\prime}}\right) \psi_{l}\right\|_{L_{t, x}^{1}} \leq & C_{l}\left(\mathbb{E}\left\|\xi-\xi_{n^{\prime}}\right\|_{L_{x}^{1}}+\mathbb{E}\left\|\xi-\xi_{n}\right\|_{L_{x}^{1}}\right) \\
& +\mathbb{E} \int_{0}^{T} \int_{0}^{t} \int_{Q}\left|A_{n}\left(u_{n}(\tau)\right)-A_{n^{\prime}}\left(u_{n^{\prime}}(\tau)\right)\right| \Delta \psi_{l} \mathrm{~d} x \mathrm{~d} \tau \mathrm{d} t \\
& +C_{l} \varepsilon^{-2} \mathbb{E}\left(\left\|\mathbf{1}_{\left|u_{n}\right| \geq n}\left(1+\left|u_{n}\right|\right)\right\|_{L_{t, x}^{m}}^{m}+\left\|\mathbf{1}_{\left|u_{n^{\prime}}\right| \geq n}\left(1+\left|u_{n^{\prime}}\right|\right)\right\|_{L_{t, x}^{m}}^{m}\right) \\
& +C_{l} \varepsilon^{-2 \nu} d\left(\sigma_{n}, \sigma_{n^{\prime}}\right)+C_{l} \varepsilon^{-2} n^{-2}+C_{l} \varepsilon^{-1} n^{-1}+M_{l}(\varepsilon) .
\end{aligned}
$$

Here the constants $C_{l}$ are independent of $\varepsilon, n, n^{\prime}$ and $M_{l}(\varepsilon)=\tilde{M}_{l}\left(\varepsilon^{2 \nu}, \varepsilon\right)$ for

$$
\tilde{M}_{l}(\delta, \varepsilon)=C_{l}\left(\delta^{2 \kappa}+\delta^{-1} \varepsilon^{2 \bar{\kappa}}+\delta \varepsilon^{-1}+\delta^{2 \alpha} \varepsilon^{-2}+\varepsilon+\sup _{|h| \leq \varepsilon} \mathbb{E}\|\xi(\cdot)-\xi(\cdot+h)\|_{L_{x}^{1}}\right) .
$$

In particular $M_{l}(\varepsilon) \rightarrow 0$ as $\varepsilon \rightarrow 0$ (for every $l$ ). Note that

$$
\begin{aligned}
\mathbb{E} \int_{0}^{T} & \int_{0}^{t} \int_{Q}\left|A_{n}\left(u_{n}(\tau)\right)-A_{n^{\prime}}\left(u_{n^{\prime}}(\tau)\right)\right| \Delta \psi_{l} \mathrm{~d} x \mathrm{~d} \tau \mathrm{d} t \\
\leq & \mathbb{E} \int_{0}^{T} \int_{0}^{t} \int_{Q}\left|A_{n}\left(u_{n}(\tau)\right)-A_{n^{\prime}}\left(u_{n^{\prime}}(\tau)\right)\right| \Delta w \mathrm{~d} x \mathrm{~d} \tau \mathrm{d} t \\
& \quad+\mathbb{E} \int_{0}^{T} \int_{0}^{t} \int_{Q}\left|A_{n}\left(u_{n}(\tau)\right)-A_{n^{\prime}}\left(u_{n^{\prime}}(\tau)\right)\right| \Delta\left(\psi_{l}-w\right) \mathrm{d} x \mathrm{~d} \tau \mathrm{d} t \\
& \lesssim \mathbb{E}\left\|\nabla\left|A_{n}\left(u_{n}\right)-A_{n^{\prime}}\left(u_{n^{\prime}}\right)\right|\right\|_{L_{t, x}^{2}}\left\|\psi_{l}-w\right\|_{H_{0 ; x}^{1}} \\
& \lesssim \mathbb{E}\left(\left\|\nabla A_{n}\left(u_{n}\right)\right\|_{L_{t, x}^{2}}+\left\|\nabla A_{n^{\prime}}\left(u_{n^{\prime}}\right)\right\|_{L_{t, x}^{2}}\right)\left\|\psi_{l}-w\right\|_{H_{0 ; x}^{1}}
\end{aligned}
$$

where in the second step we use the fact that $\Delta w=-1$, integration by parts, and the Cauchy-Schwarz inequality. By virtue of the uniform estimates (5.5) and (5.6) combined with (5.8), this gives

$$
\begin{aligned}
\mathbb{E}\left\|\left(u_{n}-u_{n^{\prime}}\right) w\right\|_{L_{t, x}^{1}} \leq & C\left\|\psi_{l}-w\right\|_{H_{0 ; x}^{1}}+C_{l}\left(\mathbb{E}\left\|\xi-\xi_{n^{\prime}}\right\|_{L_{x}^{1}}+\mathbb{E}\left\|\xi-\xi_{n}\right\|_{L_{x}^{1}}\right) \\
& +C_{l} \varepsilon^{-2} \mathbb{E}\left(\left\|\mathbf{1}_{\left|u_{n}\right| \geq n}\left(1+\left|u_{n}\right|\right)\right\|_{L_{t, x}^{m}}^{m}+\left\|\mathbf{1}_{\left|u_{n^{\prime}}\right| \geq n}\left(1+\left|u_{n^{\prime}}\right|\right)\right\|_{L_{t, x}^{m}}^{m}\right) \\
& +C_{l} \varepsilon^{-2 \nu} d\left(\sigma_{n}, \sigma_{n^{\prime}}\right)+C_{l} \varepsilon^{-2} n^{-2}+C_{l} \varepsilon^{-1} n^{-1}+M_{l}(\varepsilon),
\end{aligned}
$$

where $C$ does not depend on $l, \varepsilon, n$, or $n^{\prime}$. One can now choose first $l$ large enough and then $\varepsilon>0$ small enough so that for all $n, n^{\prime}$ large,

$$
\mathbb{E}\left\|\left(u_{n}-u_{n^{\prime}}\right) w\right\|_{L_{t, x}^{1}} \leq \frac{1}{N} .
$$

Copyright $@$ by SIAM. Unauthorized reproduction of this article is prohibited. 
Therefore, $\left(u_{n}\right)_{n \geq 1}$ converges in $L_{\omega, t}^{1} L_{w ; x}^{1}$ to a limit $u$. Moreover, by passing to a subsequence, we may also assume that

$$
\lim _{n \rightarrow \infty} u_{n}=u
$$

for almost every $(\omega, t, x) \in \Omega \times(0, T) \times Q$. Consequently, by Lemmas 5.3 and 5.4 and (5.6), we have that $u$ has the $(\star)$-property (4.1) with coefficient $\sigma$. In addition, it follows by (5.6) that for any $q<m+1$,

$$
\left(\left|u_{n}(t, x)\right|^{q}\right)_{n=1}^{\infty}
$$

is uniformly integrable on $\Omega \times[0, T] \times Q$.

We now show that $u$ is an entropy solution. From now on, when we refer to the estimate (5.5), it means that we only use it with $p=2$. By (5.6), it follows that $u$ satisfies Definition 2.4-i. Let $f \in C_{b}(\mathbb{R})$, and let $\eta$ be as in Definition 2.4. For every $n \geq 1$, we clearly have $\left[\mathfrak{a}_{n} f\right]\left(u_{n}\right) \in L_{\omega, t}^{2} H_{0, x}^{1}$ and $\partial_{x_{i}}\left[\mathfrak{a}_{n} f\right]\left(u_{n}\right)=f\left(u_{n}\right) \partial_{x_{i}}\left[\mathfrak{a}_{n}\right]\left(u_{n}\right)$. Also, we have $\left|\left[\mathfrak{a}_{n} f\right](r)\right| \leq\|f\|_{L_{r}^{\infty}} 3 K|r|^{\frac{m+1}{2}}$ for every $r \in \mathbb{R}$, which, combined with (5.5) and (5.6), gives that

$$
\sup _{n} \mathbb{E}\left\|\left[\mathfrak{a}_{n} f\right]\left(u_{n}\right)\right\|_{L_{t}^{2} H_{0 ; x}^{1}}^{2}<\infty .
$$

Hence, for a subsequence we have the weak convergences $\left[\mathfrak{a}_{n} f\right]\left(u_{n}\right) \rightarrow v_{f},\left[\mathfrak{a}_{n}\right]\left(u_{n}\right) \rightarrow v$ for some $v_{f}, v \in L_{\omega, t}^{2} H_{0 ; x}^{1}$. By (5.1), (5.9), and (5.10) it is easy to see that $v_{f}=[\mathfrak{a} f](u)$, $v=[\mathfrak{a}](u)$. Moreover, for any $\phi \in C_{c}^{\infty}([0, T) \times Q)$ and $B \in \mathcal{F}$, we have

$$
\begin{aligned}
\mathbb{E} \mathbf{1}_{B} \int_{0}^{T} \int_{Q} \partial_{x_{i}}[\mathfrak{a} f](u) \phi \mathrm{d} x \mathrm{~d} t & =\lim _{n \rightarrow \infty} \mathbb{E} \mathbf{1}_{B} \int_{0}^{T} \int_{Q} \partial_{x_{i}}\left[\mathfrak{a}_{n} f\right]\left(u_{n}\right) \phi \mathrm{d} x \mathrm{~d} t \\
& =\lim _{n \rightarrow \infty} \mathbb{E} \mathbf{1}_{B} \int_{0}^{T} \int_{Q} f\left(u_{n}\right) \partial_{x_{i}}\left[\mathfrak{a}_{n}\right]\left(u_{n}\right) \phi \mathrm{d} x \mathrm{~d} t \\
& =\mathbb{E} \mathbf{1}_{B} \int_{0}^{T} \int_{Q} f(u) \partial_{x_{i}}[\mathfrak{a}](u) \phi \mathrm{d} x \mathrm{~d} t,
\end{aligned}
$$

where for the last equality we have used that $\partial_{x_{i}}\left[\mathfrak{a}_{n}\right]\left(u_{n}\right) \rightarrow[\mathfrak{a}](u)$ (weakly) and $f\left(u_{n}\right) \rightarrow f(u)$ (strongly) in $L_{\omega, t}^{2} L_{x}^{2}$. Hence, Definition 2.4-ii is satisfied. We now show Definition 2.4-iii. Let $\eta$ and $\phi=\varphi \varrho$ be as in Definition 2.4-iii, and let $B \in \mathcal{F}$. By Itô's formula (see, e.g., [Kry13]) for the function

$$
u \mapsto \int_{Q} \eta(u) \varrho \mathrm{d} x
$$

and by Itô's product rule, we have

$$
\begin{aligned}
- & \mathbb{E} \mathbf{1}_{B} \int_{0}^{T} \int_{Q} \eta\left(u_{n}\right) \partial_{t} \phi \mathrm{d} x \mathrm{~d} t=\mathbb{E} \mathbf{1}_{B}\left[\int_{Q} \eta\left(\xi_{n}\right) \phi(0) \mathrm{d} x+\int_{0}^{T} \int_{Q}\left[\eta^{\prime} \mathfrak{a}_{n}^{2}\right]\left(u_{n}\right) \Delta \phi \mathrm{d} x \mathrm{~d} t\right. \\
& +\int_{0}^{T} \int_{Q}\left(\frac{1}{2} \phi \eta^{\prime \prime}\left(u_{n}\right)\left|\sigma_{n}\left(u_{n}\right)\right|_{\ell^{2}}^{2}-\phi \eta^{\prime \prime}\left(u_{n}\right)\left|\nabla\left[\mathfrak{a}_{n}\right]\left(u_{n}\right)\right|^{2}\right) \mathrm{d} x \mathrm{~d} t \\
& \left.+\int_{0}^{T} \int_{Q} \phi \eta^{\prime}\left(u_{n}\right) \sigma_{n}^{k}\left(u_{n}\right) \mathrm{d} x \mathrm{~d} \beta^{k}(t)\right] .
\end{aligned}
$$

Copyright $@$ by SIAM. Unauthorized reproduction of this article is prohibited. 
On the basis of (5.9) and (5.10) and the construction of $\xi_{n}, \sigma_{n}$, and $\mathfrak{a}_{n}$ it is easy to see that

$$
\begin{gathered}
\lim _{n \rightarrow \infty} \mathbb{E} \mathbf{1}_{B} \int_{Q} \eta\left(\xi_{n}\right) \phi(0) \mathrm{d} x=\mathbb{E} \mathbf{1}_{B} \int_{Q} \eta(\xi) \phi(0) \mathrm{d} x \\
\lim _{n \rightarrow \infty} \mathbb{E} \mathbf{1}_{B} \int_{0}^{T} \int_{Q} \eta\left(u_{n}\right) \partial_{t} \phi \mathrm{d} x \mathrm{~d} t=\mathbb{E} \mathbf{1}_{B} \int_{0}^{T} \int_{Q} \eta(u) \partial_{t} \phi \mathrm{d} x \mathrm{~d} t \\
\lim _{n \rightarrow \infty} \mathbb{E} \mathbf{1}_{B} \int_{0}^{T} \int_{Q}\left[\eta^{\prime} \mathfrak{a}_{n}^{2}\right]\left(u_{n}\right) \Delta \phi \mathrm{d} x \mathrm{~d} t=\mathbb{E} \mathbf{1}_{B} \int_{0}^{T} \int_{Q}\left[\eta^{\prime} \mathfrak{a}^{2}\right](u) \Delta \phi \mathrm{d} x \mathrm{~d} t, \\
\lim _{n \rightarrow \infty} \mathbb{E} \mathbf{1}_{B} \int_{0}^{T} \int_{Q} \phi \eta^{\prime \prime}\left(u_{n}\right)\left|\sigma_{n}\left(u_{n}\right)\right|_{\ell^{2}}^{2} \mathrm{~d} x \mathrm{~d} t=\mathbb{E} \mathbf{1}_{B} \int_{0}^{T} \int_{Q} \phi \eta^{\prime \prime}(u)|\sigma(u)|_{\ell^{2}}^{2} \mathrm{~d} x \mathrm{~d} t \\
\lim _{n \rightarrow \infty} \mathbb{E} \mathbf{1}_{B} \int_{0}^{T} \int_{Q} \phi \eta^{\prime}\left(u_{n}\right) \sigma_{n}^{k}\left(u_{n}\right) \mathrm{d} x \mathrm{~d} \beta^{k}(t)=\mathbb{E} \mathbf{1}_{B} \int_{0}^{T} \int_{Q} \phi \eta^{\prime}(u) \sigma^{k}(u) \mathrm{d} x \mathrm{~d} \beta^{k}(t) .
\end{gathered}
$$

Let us set $\tilde{f}(r):=\sqrt{\eta^{\prime \prime}(r)}$. Notice that $\partial_{x_{i}}\left[\tilde{f} \mathfrak{a}_{n}\right]\left(u_{n}\right)=\sqrt{\eta^{\prime \prime}\left(u_{n}\right)} \partial_{x_{i}}\left[\mathfrak{a}_{n}\right]\left(u_{n}\right)$. As before, we have (after passing to a subsequence if necessary) $\partial_{x_{i}}\left[\tilde{f} \mathfrak{a}_{n}\right]\left(u_{n}\right) \rightarrow \partial_{x_{i}}[\tilde{f} \mathfrak{a}](u)$ (weakly) in $L_{\omega, t}^{2} L_{x}^{2}$. In particular, this implies that $\partial_{x_{i}}\left[\tilde{f} \mathfrak{a}_{n}\right]\left(u_{n}\right) \rightarrow \partial_{x_{i}}[\tilde{f} \mathfrak{a}](u)$ (weakly) in $L^{2}(\Omega \times(0, T) \times Q ; \mathrm{d} \bar{\mu})$, where $\mathrm{d} \bar{\mu}:=\mathbf{1}_{B} \phi \mathrm{d} \mathbb{P} \otimes \mathrm{d} x \otimes \mathrm{d} t$. This implies that

$$
\mathbb{E} \mathbf{1}_{B} \int_{0}^{T} \int_{Q} \phi \eta^{\prime \prime}(u)|\nabla[\mathfrak{a}](u)|^{2} \mathrm{~d} x \mathrm{~d} t \leq \liminf _{n \rightarrow \infty} \mathbb{E} \mathbf{1}_{B} \int_{0}^{T} \int_{Q} \phi \eta^{\prime \prime}\left(u_{n}\right)\left|\nabla\left[\mathfrak{a}_{n}\right]\left(u_{n}\right)\right|^{2} \mathrm{~d} x \mathrm{~d} t .
$$

Hence, taking lim inf in (5.11) along an appropriate subsequence, we see that $u$ satisfies Definition 2.4-iii too.

To summarize, we have shown that if, in addition to the assumptions of Theorem 3.1, we have that $\mathbb{E}\|\xi\|_{L_{x}^{2}}^{4}<\infty$, then there exists an entropy solution to (2.1) which has the ( $\star$ )-property (4.1) with coefficient $\sigma$ (therefore, it is also unique by (4.19) in Corollary 4.5). In addition, we can pass to the limit in (5.5) and (5.6) to obtain that

$$
\begin{gathered}
\mathbb{E} \sup _{t \leq T}\|u\|_{L_{x}^{2}}^{2}+\mathbb{E}\|\nabla[\mathfrak{a}](u)\|_{L_{t, x}^{2}}^{2} \lesssim 1+\mathbb{E}\|\xi\|_{L_{x}^{2}}^{2}, \\
\mathbb{E} \sup _{t \leq T}\|u\|_{L_{t, x}^{m+1}}^{m+1}+\mathbb{E}\|\nabla A(u)\|_{L_{t, x}^{2}}^{2} \lesssim 1+\mathbb{E}\|\xi\|_{L_{x}^{m+1}}^{m+1} .
\end{gathered}
$$

Step 2. We now remove the extra condition on $\xi$. For $n \geq 1$, let $\xi_{n}$ be as in (5.2), and let $u_{(n)}$ be the unique solution of $\mathcal{E}\left(A, \sigma, \xi_{n}\right)$. Note that $u_{(n)}$ has the ( $\star$ )-property with coefficient $\sigma$. Hence, by equation (4.19) we have that $\left(u_{(n)}\right)_{n \geq 1}$ is Cauchy in $L_{\omega, t}^{1} L_{w ; x}^{1}$ and therefore has a limit $u$. In addition, $u_{(n)}$ satisfy the estimates (5.12) and (5.13) uniformly in $n$. With the arguments provided in Step 1 we can show that $u$ is an entropy solution.

Step 3 . We finally show (3.1), which also implies uniqueness. Let $\tilde{u}$ be an entropy solution of $\mathcal{E}(A, \sigma, \tilde{\xi})$. By (4.19) we have that

$$
\sup _{t \in[0, T]} \operatorname{ess} \mathbb{E}\left\|u_{(n)}(t)-\tilde{u}(t)\right\|_{L_{w ; x}^{1}} \leq \mathbb{E}\left\|\xi_{n}-\tilde{\xi}\right\|_{L_{w ; x}^{1}}
$$

for the sequence $u_{(n)}$ as in Step 2. The proof is complete if we let $n \rightarrow \infty$.

Remark 5.6. Let the assumptions of Theorem 3.1 hold. If we further assume that $\inf _{r \geq 0} \mathfrak{a}(r)=c>0$, it is easy to see that, in addition to (5.12) and (5.13), we have

$$
\mathbb{E}\|u\|_{L_{t}^{2} H_{0 ; x}^{1}}^{2} \leq C\left(1+\mathbb{E}\|\xi\|_{L_{x}^{2}}^{2}\right)
$$

Copyright (c) by SIAM. Unauthorized reproduction of this article is prohibited. 
with $C$ depending on $\mathcal{S}$ and $c$. Furthermore, after a standard approximation argument, it follows from Definition 2.4 that for each $\phi \in H_{0 ; x}^{1}$ we have

$$
(u(t), \phi)=(\xi, \phi)-\int_{0}^{t}(\nabla A(u(s)), \nabla \phi) d s+\int_{0}^{t}\left(\sigma^{k}(u(s)), \phi\right) \mathrm{d} \beta^{k}(s)
$$

for almost every $(\omega, t)$. These two facts imply by virtue of [KR79, Theorem 3.2] that $u$ is a continuous $L_{x}^{2}$-valued process.

We now proceed with the proof of Theorem 3.2 which implies the stability of entropy solutions with respect to the initial condition $\xi$, the nonlinearity $A$, and $\sigma$. The proof is similar to [DGG19, Proof of Theorem 2.2].

Proof of Theorem 3.2. Let $\bar{\xi}_{n}=-a_{n} \vee\left(a_{n} \wedge \xi_{n}\right)$, where $a_{n}$ is chosen large enough such that

$$
\mathbb{E}\left\|\xi_{n}-\bar{\xi}_{n}\right\|_{L_{w ; x}^{1}} \leq \frac{1}{n}
$$

Let $\bar{u}_{n}$ be the solution of $\mathcal{E}\left(A_{n}, \sigma_{n}, \bar{\xi}_{n}\right)$ which, by Theorem 3.1, exists and is unique and by Lemma 5.4 satisfies the $(\star)$-property (4.1). By (4.19) we know that

$$
\mathbb{E}\left\|u_{n}-\bar{u}_{n}\right\|_{L_{t}^{1} L_{w ; x}^{1}} \leq \mathbb{E} \int_{0}^{T}\left\|\xi_{n}-\bar{\xi}_{n}\right\|_{L_{w ; x}^{1}} \mathrm{~d} t \leq \frac{T}{n} .
$$

Thus, it is enough to prove that $\bar{u}_{n} \rightarrow u$ in $L_{\omega, t}^{1} L_{w ; x}^{1}$. Let $\psi_{l} \in C_{c}^{\infty}(Q)$ be a sequence of positive functions such that $\left\|\psi_{l}-w\right\|_{H_{0 ; x}^{1}} \rightarrow 0$ as $l \rightarrow \infty$. Then, for arbitrary $l \geq 1$, we have that

$$
\begin{aligned}
\mathbb{E}\left\|\bar{u}_{n}-u\right\|_{L_{t}^{1} L_{w ; x}^{1}} & \leq \mathbb{E}\left\|\left(\bar{u}_{n}-u\right) \psi_{l}\right\|_{L_{t}^{1} L_{x}^{1}}+\mathbb{E}\left\|\left(\bar{u}_{n}-u\right)\left(w-\psi_{l}\right)\right\|_{L_{t}^{1} L_{x}^{1}} \\
& \leq \mathbb{E}\left\|\left(\bar{u}_{n}-u\right) \psi_{l}\right\|_{L_{t}^{1} L_{x}^{1}}+C\left\|w-\psi_{l}\right\|_{H_{0 ; x}^{1}}
\end{aligned}
$$

for some $C$ independent on $n$ (similarly to (5.12)) and $l$. Using Lemma 4.4-1 and proceeding similarly to Step 1 in the proof of Theorem 3.1, where the specific choice of $\lambda=\frac{8}{n}$ implies that $R_{\lambda} \geq b_{n}$ for some $b_{n} \geq 1$ which can be chosen such that $b_{n} \rightarrow \infty$ as $n \rightarrow \infty$ (since $A_{n} \rightarrow A$ uniformly on compact sets by assumption), we obtain that

$$
\begin{aligned}
\mathbb{E}\left\|\bar{u}_{n}-u\right\|_{L_{t}^{1} L_{w ; x}^{1}} \leq & C\left\|w-\psi_{l}\right\|_{H_{0, x}^{1}}+C_{l} \mathbb{E}\left\|\bar{\xi}_{n}-\xi\right\|_{L_{x}^{1}} \\
& +C_{l} \varepsilon^{-2} \mathbb{E}\left(\left\|\mathbf{1}_{\left|\bar{u}_{n}\right| \geq b_{n}}\left(1+\left|\bar{u}_{n}\right|\right)\right\|_{L_{t, x}^{m}}^{m}+\left\|\mathbf{1}_{|u| \geq b_{n}}(1+|u|)\right\|_{L_{t, x}^{m}}^{m}\right) \\
& +C_{l} \varepsilon^{-2 \nu} d\left(\sigma_{n}, \sigma\right)+C_{l} \varepsilon^{-2} n^{-2}+C_{l} \varepsilon^{-1} n^{-1}+M_{l}(\varepsilon)
\end{aligned}
$$

for some $M_{l}(\varepsilon) \rightarrow 0$ as $\varepsilon \rightarrow 0$ (for fixed $l$ ) and constants $C_{l}$ independent of $\varepsilon, n$, and $C$ independent of $\varepsilon, l, n$. To conclude, given $N \geq 1$ we first choose $l$ large enough and then $\varepsilon$ small enough so that for all $n$ large enough,

$$
\mathbb{E}\left\|\bar{u}_{n}-u\right\|_{L_{t}^{1} L_{w ; x}^{1}} \leq \frac{1}{N},
$$

which completes the proof.

5.2. Proofs of Theorem 3.3 and Proposition 3.4. To prove that entropy solutions belong to $C\left([0, T] ; L_{\omega}^{1} L_{w ; x}^{1}\right)$, we use the continuity of vanishing viscosity approximations from Proposition 5.5 and the stability theorem, Theorem 3.2, together with Lemma 4.3 .

Copyright $@$ by SIAM. Unauthorized reproduction of this article is prohibited. 
Proof of Theorem 3.3. Let $u_{n}$ be given by Proposition 5.5. Recall that by Theorem 3.2 we have

$$
\lim _{n \rightarrow 0} \mathbb{E}\left\|u-u_{n}\right\|_{L_{t}^{1} L_{w ; x}^{1}}=0,
$$

which in particular implies that there exists $\mathcal{T} \subset[0, T]$ with $|\mathcal{T}|=T$ and a (nonrelabeled) subsequence such that

$$
\lim _{n \rightarrow \infty} \mathbb{E}\left\|u(t)-u_{n}(t)\right\|_{L_{w ; x}^{1}}=0
$$

for every $t \in \mathcal{T}$. We now show that given $\lambda>0$, there exists $h$ such that

$$
\mathbb{E}\left\|u_{n}(t)-u_{n}\left(t^{\prime}\right)\right\|_{L_{w ; x}^{1}} \leq \lambda
$$

for every $n \geq 1$ and $t, t^{\prime} \in \mathcal{T}$ with $\left|t-t^{\prime}\right| \leq h$. By (5.6) we have

$$
\mathbb{E}\left\|u_{n}(t)-u_{n}\left(t^{\prime}\right)\right\|_{L_{w ; x}^{1}} \lesssim \xi\left\|w-\psi_{l}\right\|_{L_{x}^{2}}+\mathbb{E} \int_{Q}\left|u_{n}(t)-u_{n}\left(t^{\prime}\right)\right| \psi_{l} \mathrm{~d} x,
$$

where $\psi_{l} \in C_{c}^{\infty}(Q), \psi_{l} \geq 0$, and $\left\|w-\psi_{l}\right\|_{H_{0 ; x}^{1}} \rightarrow 0$. We also have

$$
\begin{aligned}
& \mathbb{E} \int_{Q}\left|u_{n}(t, x)-u_{n}\left(t^{\prime}, x\right)\right| \psi_{l}(x) d x \\
& \leq \mathbb{E} \int_{Q} \int_{Q}\left|u_{n}(t, x)-u_{n}\left(t^{\prime}, y\right)\right| \psi_{l}(x) \varrho_{\varepsilon}(x-y) \mathrm{d} x \mathrm{~d} y \quad\left(=: I_{1}\right) \\
& \quad+\mathbb{E} \int_{Q} \int_{Q}\left|u_{n}\left(t^{\prime}, x\right)-u_{n}\left(t^{\prime}, y\right)\right| \psi_{l}(x) \varrho_{\varepsilon}(x-y) \mathrm{d} x \mathrm{~d} y . \quad\left(=: I_{2}\right)
\end{aligned}
$$

By Lemma 4.3 and using that $u_{n}$ is continuous in $t$ with values in $L_{x}^{2}$ (see Remark 5.6) for every $n \geq 1$ (hence every $t \in[0, T]$ is a Lebesgue point of (4.13)), we obtain

$$
\begin{aligned}
I_{2}= & \mathbb{E} \int_{Q} \int_{Q}\left|u_{n}\left(t^{\prime}, x\right)-u_{n}\left(t^{\prime}, y\right)\right| \psi_{l}(x) \varrho_{\varepsilon}(x-y) \mathrm{d} x \mathrm{~d} y \\
\leq & \mathbb{E} \int_{Q} \int_{Q}\left|\xi_{n}(x)-\xi_{n}(y)\right| \psi_{l}(x) \varrho_{\varepsilon}(x-y) \mathrm{d} x \mathrm{~d} y \\
& +\mathbb{E} \int_{0}^{t^{\prime}} \int_{Q^{2}}\left|A_{n}\left(u_{n}(s, x)\right)-A_{n}\left(u_{n}(s, y)\right)\right| \Delta \psi_{l}(x) \varrho_{\varepsilon}(x-y) \mathrm{d} x \mathrm{~d} y \mathrm{~d} t \\
& +C \mathcal{G}_{\alpha}(\delta, \varepsilon, 0) \mathbb{E}\left(1+\left\|u_{n}\right\|_{L_{t, x}^{m+1}}^{m+1}\right) \\
\leq & \mathbb{E} \int_{Q} \int_{Q}|\xi(x)-\xi(y)| \psi_{l}(x) \varrho_{\varepsilon}(x-y) \mathrm{d} x \mathrm{~d} y \\
& +C \varepsilon \mathbb{E}\left\|\nabla A_{n}\left(u_{n}\right)\right\|_{L_{t, x}^{1}}\left\|\Delta \psi_{l}\right\|_{L_{x}^{\infty}}+C_{l} \mathcal{G}_{\alpha}(\delta, \varepsilon, 0) \mathbb{E}\left(1+\left\|u_{n}\right\|_{L_{t, x}^{m+1}}^{m+1}\right)
\end{aligned}
$$

for some constant $C_{l}>0$ which depends on $l$ but not on $\delta$ and $\varepsilon$. Using (5.6) we conclude that

$$
I_{2} \lesssim \xi \mathbb{E} \int_{Q} \int_{Q}|\xi(x)-\xi(y)| \psi_{l}(x) \varrho_{\varepsilon}(x-y) \mathrm{d} x \mathrm{~d} y+\varepsilon\left\|\Delta \psi_{l}\right\|_{L_{x}^{\infty}}+M_{l}(\varepsilon),
$$

Copyright $@$ by SIAM. Unauthorized reproduction of this article is prohibited. 
where $M_{l}(\varepsilon) \rightarrow 0$ as $\varepsilon \rightarrow 0$ (for fixed $l$, by choosing $\delta$ and $\alpha$ as in the proof of Lemma 4.4-2). We also have that

$$
I_{1} \lesssim \delta\left\|\psi_{l}\right\|_{L_{x}^{\infty}}+\mathbb{E} \int_{Q} \int_{Q} \eta_{\delta}\left(u_{n}(t, x)-u_{n}\left(t^{\prime}, y\right)\right) \psi_{l}(x) \varrho_{\varepsilon}(x-y) \mathrm{d} x \mathrm{~d} y .
$$

By Itô's formula we conclude that

$$
\begin{aligned}
& \mathbb{E} \int_{Q} \int_{Q} \eta_{\delta}\left(u_{n}(t, x)-u_{n}\left(t^{\prime}, y\right)\right) \psi_{l}(x) \varrho_{\varepsilon}(x-y) \mathrm{d} x \mathrm{~d} y \\
& \leq \\
& \quad \mathbb{E} \int_{Q} \int_{Q} \eta_{\delta}\left(u_{n}\left(t^{\prime}, x\right)-u_{n}\left(t^{\prime}, y\right)\right) \psi_{l}(x) \varrho_{\varepsilon}(x-y) \mathrm{d} x \mathrm{~d} y \\
& \quad+\mathbb{E} \int_{t^{\prime}}^{t} \int_{Q} \int_{Q} \eta_{\delta}^{\prime}\left(u_{n}(s, x)-u_{n}\left(t^{\prime}, y\right)\right) \nabla A_{n}\left(u_{n}(s, x)\right) \nabla\left(\psi_{l}(x) \varrho_{\varepsilon}(x-y)\right) \mathrm{d} x \mathrm{~d} y \mathrm{~d} s \\
& \quad+\mathbb{E} \int_{t^{\prime}}^{t} \int_{Q} \int_{Q} \frac{1}{2} \eta_{\delta}^{\prime \prime}\left(u_{n}(s, x)-u_{n}\left(t^{\prime}, y\right)\right)\left|\sigma_{n}\left(x, u_{n}(s, x)\right)\right|_{\ell^{2}}^{2} \psi_{l}(x) \varrho_{\varepsilon}(x-y) \mathrm{d} x \mathrm{~d} y \mathrm{~d} s,
\end{aligned}
$$

where we have used the fact that

$-\mathbb{E} \int_{t^{\prime}}^{t} \int_{Q} \int_{Q} \eta_{\delta}^{\prime \prime}\left(u_{n}(s, x)-u_{n}\left(t^{\prime}, y\right)\right) \nabla u_{n}(s, x) \nabla A_{n}\left(u_{n}(s, x)\right) \psi_{l}(x) \varrho_{\varepsilon}(x-y) \mathrm{d} x \mathrm{~d} y \mathrm{~d} s \leq 0$.

From this, using the fact that $\left|\sigma_{n}\left(x, u_{n}(s, x)\right)\right|_{\ell^{2}}^{2} \lesssim 1+\left|u_{n}(s, x)\right|^{2}$, it follows that

$$
\begin{aligned}
I_{1} & \lesssim \delta\left\|\psi_{l}\right\|_{L_{x}^{\infty}}+I_{2}+\left\|\psi_{l}\right\|_{W_{x}^{1, \infty}} \int_{t^{\prime}}^{t} \varepsilon^{-1} \mathbb{E}\left\|\nabla A_{n}\left(u_{n}(s)\right)\right\|_{L_{x}^{2}}+\delta^{-1}\left(1+\mathbb{E}\left\|u_{n}(s)\right\|_{L_{x}^{2}}^{2}\right) \mathrm{d} s \\
& \lesssim \delta\left\|\psi_{l}\right\|_{L_{x}^{\infty}}+I_{2}+\varepsilon^{-1}\left|t^{\prime}-t\right|^{\frac{1}{2}} \mathbb{E}\left\|\nabla A_{n}\left(u_{n}\right)\right\|_{L_{t, x}^{2}}+\delta^{-1}\left|t^{\prime}-t\right|^{\frac{m-1}{m+1}}\left(1+\mathbb{E}\left\|u_{n}(s)\right\|_{L_{t, x}^{m+1}}^{2}\right) \\
& \lesssim \xi\left\|\psi_{l}\right\|_{L_{x}^{\infty}}+I_{2}+\varepsilon^{-1}\left|t^{\prime}-t\right|^{\frac{1}{2}}+\delta^{-1}\left|t^{\prime}-t\right|^{\frac{m-1}{m+1}} .
\end{aligned}
$$

Consequently, by (5.15)-(5.18), we obtain

$$
\begin{aligned}
& \mathbb{E}\left\|u_{n}(t)-u_{n}\left(t^{\prime}\right)\right\|_{L_{w ; x}^{1}} \\
& \lesssim_{\xi}\left\|w-\psi_{l}\right\|_{L_{x}^{2}}+\mathbb{E} \int_{Q} \int_{Q}|\xi(x)-\xi(y)| \psi_{l}(x) \varrho_{\varepsilon}(x-y) \mathrm{d} x \mathrm{~d} y+\varepsilon\left\|\Delta \psi_{l}\right\|_{L_{x}^{\infty}} \\
& \quad+M_{l}(\varepsilon)+\delta\left\|\psi_{l}\right\|_{L_{x}^{\infty}}+\varepsilon^{-1}\left|t^{\prime}-t\right|^{\frac{1}{2}}+\delta^{-1}\left|t^{\prime}-t\right|^{\frac{m-1}{m+1}} .
\end{aligned}
$$

Then, given $\lambda>0$, we choose $l$ large enough and $\varepsilon, \delta>0$ small enough so that

$$
\begin{array}{r}
\left\|w-\psi_{l}\right\|_{L_{x}^{2}}+\mathbb{E} \int_{Q} \int_{Q}|\xi(x)-\xi(y)| \psi_{l}(x) \varrho_{\varepsilon}(x-y) \mathrm{d} x \mathrm{~d} y \\
+\varepsilon\left\|\Delta \psi_{l}\right\|_{L_{x}^{\infty}}+M_{l}(\varepsilon)+\delta\left\|\psi_{l}\right\|_{L_{x}^{\infty}} \leq \lambda / 2 .
\end{array}
$$

Then it is clear that for every $t^{\prime}, t \in \mathcal{T}$ such that $\left|t^{\prime}-t\right|$ is sufficiently small, we have

$$
\mathbb{E}\left\|u_{n}(t)-u_{n}\left(t^{\prime}\right)\right\|_{L_{w ; x}^{1}} \leq \lambda,
$$

which, after passing to the limit $n \rightarrow \infty$, gives

$$
\mathbb{E}\left\|u(t)-u\left(t^{\prime}\right)\right\|_{L_{w ; x}^{1}} \leq \lambda .
$$

Consequently $u: \mathcal{T} \rightarrow L_{\omega}^{1} L_{w ; x}^{1}$ is uniformly continuous, and hence it has a unique continuous extension on $[0, T]$.

Copyright $@$ by SIAM. Unauthorized reproduction of this article is prohibited. 
Proof of Proposition 3.4. Let $\left(\xi_{n}\right)_{n \geq 1}$ be a sequence in $L_{\omega}^{m+1} L_{x}^{m+1}$ such that $\xi_{n} \rightarrow$ $\xi$ in $L_{\omega}^{1} L_{w ; x}^{1}$. By Theorem 3.1, for every $m>n \geq 1$ we have the estimate

$$
\sup _{t \in[0, T]} \mathbb{E}\left\|u\left(t ; \xi_{m}\right)-u\left(t ; \xi_{n}\right)\right\|_{L_{w ; x}^{1}} \leq \mathbb{E}\left\|\xi_{m}-\xi_{n}\right\|_{L_{w ; x}^{1}},
$$

where we have replaced supess $\operatorname{es}_{t \in[0, T]}$ by $\sup _{t \in[0, T]}$ in (3.1) since, by Theorem 3.3, we know that $u\left(\cdot ; \xi_{n}\right) \in C\left([0, T] ; L_{\omega}^{1} L_{w ; x}^{1}\right)$ for every $n \geq 1$. Thus, the sequence $\left\{u\left(\cdot ; \xi_{n}\right)\right\}_{n \geq 1}$ is Cauchy in $C\left([0, T] ; L_{\omega}^{1} L_{w ; x}^{1}\right)$, which implies that the limit $v(\cdot ; \xi):=$ $\lim _{n \rightarrow \infty} u\left(\cdot ; \xi_{n}\right)$ exists in $C\left([0, T] ; L_{\omega}^{1} L_{w ; x}^{1}\right)$. Moreover, if $\left(\tilde{\xi}_{n}\right)_{n>1}$ is another sequence in $L_{\omega}^{m+1} L_{x}^{m+1}$ which converges to $\xi$ in $L_{\omega}^{1} L_{w ; x}^{1}$, by again using Theorem 3.1 we get that for every $n \geq 1$,

$$
\sup _{t \in[0, T]} \mathbb{E}\left\|u\left(t ; \xi_{n}\right)-u\left(t ; \tilde{\xi}_{n}\right)\right\|_{L_{w ; x}^{1}} \leq \mathbb{E}\left\|\xi_{n}-\tilde{\xi}_{n}\right\|_{L_{w ; x}^{1},}
$$

which shows that $v(\cdot ; \xi)$ is independent of $\left(\xi_{n}\right)_{n \geq 1}$. It is easy to see that for $\xi \in L_{\omega}^{m+1} L_{x}^{m+1}, v(\cdot ; \xi)$ coincides with $u(\cdot ; \xi)$ in $C\left([0, T] ; L_{\omega}^{1} L_{w ; x}^{1}\right)$. Thus, $v$ is the unique continuous (with respect to the variable $\xi$ ) extension of $u$ on $C\left([0, T] ; L_{\omega}^{1} L_{w ; x}^{1}\right)$. To ease the notation we identify $u$ with $v$. Finally, (3.2) follows easily by construction.

\section{Proofs of ergodicity.}

6.1. Proof of Proposition 3.6. This is a simple application of Fatou's lemma combined with Lemma A.1 and Theorem 3.3.

Proof of Proposition 3.6. Let $t \in[0, \infty)$. By Theorem 3.3 we know that $u(\cdot ; \xi) \in$ $C\left([0, \infty) ; L_{\omega}^{1} L_{w ; x}^{1}\right)$, and hence there exists a sequence $t_{n} \rightarrow t$ such that $u\left(t_{n} ; \xi\right) \rightarrow$ $u(t ; \xi)$ for almost every $(\omega, x)$. By Lemma A.1 we can assume that

$$
\sup _{n \geq 1}\left(t_{n} \wedge 1\right)^{\frac{m+1}{m-1}} \mathbb{E}\left\|u\left(t_{n} ; \xi\right)\right\|_{L_{x}^{m+1}}^{m+1} \leq C
$$

for some $C>0$ depending only on $d, K, m$, and $|Q|$ and not on $t$ and $\xi$. Then, by Fatou's lemma we have that

$$
\begin{aligned}
(t \wedge 1)^{\frac{m+1}{m-1}} \mathbb{E}\|u(t ; \xi)\|_{L_{x}^{m+1}}^{m+1} & \leq \liminf _{n \rightarrow \infty}\left(t_{n} \wedge 1\right)^{\frac{m+1}{m-1}} \mathbb{E}\left\|u\left(t_{n} ; \xi\right)\right\|_{L_{x}^{m+1}}^{m+1} \\
& \leq \sup _{n \geq 1}\left(t_{n} \wedge 1\right)^{\frac{m+1}{m-1}} \mathbb{E}\left\|u\left(t_{n} ; \xi\right)\right\|_{L_{x}^{m+1}}^{m+1} \leq C,
\end{aligned}
$$

which completes the proof since $t$ is arbitrary.

6.2. Proof of Theorem 3.8. In this section we prove Theorem 3.8. The proof is based on (4.18) in Corollary 4.5, which, however, we have only shown for entropy solutions satisfying the $(\star)$-property $(4.1)$ and for almost every $s<t$. For this reason we first use the approximations from Proposition 5.5 which satisfy the $(\star)$-property and pass to the limit in (4.18). We then use the continuity of solutions in $L_{\omega}^{1} L_{w ; x}^{1}$ given by Theorem 3.3 to derive the estimate for every $s<t$.

Proof of Theorem 3.8. We first assume that $\xi, \tilde{\xi} \in L_{\omega}^{m+1} L_{x}^{m+1}$. By Theorem 3.2 we can approximate $u(\cdot ; \xi), u(\cdot ; \tilde{\xi})$ in $L_{\omega, t}^{1} L_{w ; x}^{1}$ by $u_{n}, \tilde{u}_{n}$ as in Proposition 5.5 satisfying the ( $\star$ )-property. By (4.18) in Corollary 4.5 and by passing to the limit $n \rightarrow \infty$ (upon a subsequence) we obtain that for almost every $0 \leq s<t \leq T$ (including $s=0$ ), 


$$
\begin{aligned}
& \mathbb{E}\|u(t ; \xi)-u(t ; \tilde{\xi})\|_{L_{w ; x}^{1}}-\mathbb{E}\|u(s ; \xi)-u(s ; \tilde{\xi})\|_{L_{w ; x}^{1}} \\
& \quad \leq-\mathbb{E} \int_{s}^{t}\left\||u(\tau ; \xi)|^{m-1} u(\tau ; \xi)-|u(\tau ; \tilde{\xi})|^{m-1} u(\tau ; \tilde{\xi})\right\|_{L_{x}^{1}} \mathrm{~d} \tau .
\end{aligned}
$$

Since by Theorem 3.3 we know that $u(\cdot ; \xi), u(\cdot ; \tilde{\xi}) \in C\left([0, T] ; L_{\omega}^{1} L_{w ; x}^{1}\right)$, the same estimate holds for every $0 \leq s<t \leq T$. Combining this estimate with Lemma B.1, there exists $C>0$ depending only on $m$ such that

$\mathbb{E}\|u(t ; \xi)-\tilde{u}(t ; \tilde{\xi})\|_{L_{w ; x}^{1}}-\mathbb{E}\|u(s ; \xi)-u(s ; \tilde{\xi})\|_{L_{w ; x}^{1}} \leq-C \int_{s}^{t} \mathbb{E}\|u(\tau ; \xi)-u(\tau ; \tilde{\xi})\|_{L_{x}^{m}}^{m} \mathrm{~d} \tau$,

and if we furthermore note that

$$
\left(\mathbb{E}\|u(\tau ; \xi)-u(\tau ; \tilde{\xi})\|_{L_{w ; x}^{1}}\right)^{m} \leq \mathbb{E}\|u(\tau ; \xi)-u(\tau ; \tilde{\xi})\|_{L_{x}^{m}}^{m}\|w\|_{L_{x}^{m *}}^{m},
$$

we finally obtain the integral inequality

$$
\begin{aligned}
& \mathbb{E}\|u(t ; \xi)-u(t ; \tilde{\xi})\|_{L_{w ; x}^{1}}-\mathbb{E}\|u(s ; \xi)-u(s ; \tilde{\xi})\|_{L_{w ; x}^{1}} \\
& \leq-C\|w\|_{L_{x}^{m *}}^{-m} \int_{s}^{t}\left(\mathbb{E}\|u(\tau ; \xi)-u(\tau ; \tilde{\xi})\|_{L_{w ; x}^{1}}\right)^{m} \mathrm{~d} \tau .
\end{aligned}
$$

Let $f(t)=\mathbb{E}\|u(t ; \xi)-u(t ; \tilde{\xi})\|_{L_{w ; x}^{1}}$. Then $f$ satisfies the integral inequality

$$
f(t)-f(s) \leq-C\|w\|_{L_{x}^{m *}}^{-m} \int_{s}^{t} f(\tau)^{m} \mathrm{~d} \tau
$$

for every $s \leq t$, and it is continuous since $u, \tilde{u} \in C\left([0, T] ; L_{\omega}^{1} L_{w ; x}^{1}\right)$. Hence, by Lemma B.2 we obtain that $f(t) \leq h(t)$, where $h$ solves

$$
\left\{\begin{array}{l}
h^{\prime}(t)=-C\|w\|_{L_{x}^{m *}}^{-m} h(t)^{m} \\
h(0)=f(0) .
\end{array}\right.
$$

A simple computation shows that

$$
h(t)=\left(\frac{1}{h(0)^{-(m-1)}+C\|w\|_{L_{x}^{m_{*}}}^{-m_{*}}(m-1) t}\right)^{\frac{1}{m-1}},
$$

which, in turn, implies that

$$
\mathbb{E}\|u(t ; \xi)-u(t ; \tilde{\xi})\|_{L_{w ; x}^{1} \leq} \leq C\|w\|_{L_{x}^{m *}}^{\frac{m}{m-1}} t^{-\frac{1}{m-1}} .
$$

If $\xi, \tilde{\xi} \in L_{\omega}^{1} L_{w ; x}^{1}$, we approximate with sequences $\left(\xi_{n}\right)_{n \geq 1},\left(\tilde{\xi}_{n}\right)_{n \geq 1}$ in $L_{\omega}^{m+1} L_{x}^{m+1}$ and use (3.2) to pass to the limit.

6.3. Proofs of Proposition 3.10 and Theorem 3.12. To prove Proposition 3.10 , we first prove the flow property for entropy solutions (see Corollary 6.1). This uses the continuity of solutions in $L_{\omega}^{1} L_{w ; x}^{1}$ given by Theorem 3.3 in combination with an approximation argument in $L_{\omega}^{m+1} L_{x}^{m+1}$ in the spirit of [DPZ14, Theorem 9.14], which strongly relies on the weighted $L^{1}$-contraction estimate (3.2). For technical reasons, in this section we extend the time horizon to $-\infty$.

Copyright $@$ by SIAM. Unauthorized reproduction of this article is prohibited. 
We need the following notation. For $\xi \in L_{x}^{m+1}$ and $s>-\infty$ we denote by $u_{s}(\cdot ; \xi)$ the entropy solution to

$$
\left\{\begin{array}{l}
\partial_{t} u_{s}(t, x ; \xi)=\Delta\left(\left|u_{s}(t ; \xi)\right|^{m-1} u_{s}(t ; \xi)\right)(t, x)+\sigma^{k}\left(x, u_{s}(t, x ; \xi)\right) \dot{\beta}^{k}(t), \\
u_{s}(s, x ; \xi)=\xi \\
\left.u_{s}\right|_{\partial Q}=0
\end{array}\right.
$$

for $t \geq s$, where we have extended $\beta^{k}(t)$ for $t<0$ by gluing at $t=0$ an independent Brownian motion evolving backwards in time. The existence and uniqueness of entropy solutions to this equation for $s=0$ are given by Theorem 3.1. The case $s \neq 0$ follows analogously. To be consistent with the previous sections, we simply write $u(\cdot ; \xi)$ to denote $u_{0}(\cdot ; \xi)$.

We have the following useful relation.

Corollary 6.1. For every $\xi \in L_{x}^{m+1}$ and $-\infty<s_{1} \leq s_{2} \leq t \leq T$ we have that $u_{s_{1}}(t ; \xi)=u_{s_{2}}\left(t ; u_{s_{1}}\left(s_{2} ; \xi\right)\right)$ in $L_{\omega}^{1} L_{w ; x}^{1}$.

Proof. For simplicity we prove the statement for $\left(s_{1}, s_{2}\right)=(0, s)$. If $u$ is an entropy solution on $[0, T]$ and $\varphi \in C_{c}^{\infty}([s, T)$ ), which can be extended continuously on $[0, s]$ (taking the constant value $\varphi(s)$ ), we can choose $\phi=\chi_{k} \varphi \varrho$ in Definition 2.4 with

$$
\chi_{\kappa}(t)= \begin{cases}1, & t \in[s, T), \\ \kappa\left(t-\left(s-\frac{1}{\kappa}\right)\right), & t \in\left[s-\frac{1}{\kappa}, s\right], \\ 0, & t \in\left[0, s-\frac{1}{\kappa}\right]\end{cases}
$$

to obtain that

$$
\begin{aligned}
& -\int_{0}^{T} \int_{Q} \eta(u) \chi_{\kappa} \partial_{t} \varphi(t) \varrho \mathrm{d} x \mathrm{~d} t \leq \kappa \int_{s-\frac{1}{\kappa}}^{s} \int_{Q} \eta(u) \varphi(s) \varrho \mathrm{d} x \mathrm{~d} t \\
& \quad+\int_{0}^{T} \int_{Q}\left[\eta^{\prime} \mathfrak{a}^{2}\right](u) \chi_{\kappa} \varphi \Delta \rho \mathrm{d} x \mathrm{~d} t \\
& \quad+\int_{0}^{T} \int_{Q}\left(\frac{1}{2} \chi_{\kappa} \varphi \varrho \eta^{\prime \prime}(u)|\sigma(u)|_{\ell^{2}}^{2}-\chi_{\kappa} \varphi \varrho \eta^{\prime \prime}(u)|\nabla[\mathfrak{a}](u)|^{2}\right) \mathrm{d} x \mathrm{~d} t \\
& \quad+\int_{0}^{T} \int_{Q} \chi_{\kappa} \varphi \varrho \eta^{\prime}(u) \sigma^{k}(u) \mathrm{d} x \mathrm{~d} \beta^{k}(t) .
\end{aligned}
$$

One can prove that $u(s) \in L_{\omega}^{m+1} L_{x}^{m+1}$. Indeed, we can approximate $u$ by $u_{n}$ as in Proposition 5.5 in $L_{\omega, t}^{1} L_{w ; x}^{1}$, and hence for almost every $(\omega, t, x)$ up to a subsequence, and use (5.6) and Fatou's lemma to obtain that

$$
\sup _{t \in[0, T]} \operatorname{ess}\|u(t)\|_{L_{x}^{m+1}}^{m+1} \lesssim 1+\mathbb{E}\|\xi\|_{L_{x}^{m+1}}^{m+1} .
$$

This estimate, together with the fact that $u \in C\left([0, T] ; L_{\omega}^{1} L_{w ; x}^{1}\right)$, implies that there exists a sequence $s_{n} \rightarrow s$ such that $u\left(s_{n}, x\right) \rightarrow u(s, x)$ for almost every $(\omega, x)$ and

$$
\sup _{n \geq 1} \mathbb{E}\left\|u\left(s_{n}\right)\right\|_{L_{x}^{m+1}}^{m+1} \lesssim 1+\mathbb{E}\|\xi\|_{L_{x}^{m+1}}^{m+1}
$$

Then, again by Fatou's lemma, we have that

$$
\mathbb{E}\|u(s)\|_{L_{x}^{m+1}}^{m+1} \lesssim \liminf _{n \rightarrow \infty} \mathbb{E}\left\|u\left(s_{n}\right)\right\|_{L_{x}^{m+1}}^{m+1} \lesssim \sup _{n \geq 1} \mathbb{E}\left\|u\left(s_{n}\right)\right\|_{L_{x}^{m+1}}^{m+1} \lesssim 1+\mathbb{E}\|\xi\|_{L_{x}^{m+1}}^{m+1},
$$


which implies that $u(s) \in L_{\omega}^{m+1} L_{x}^{m+1}$. We also have the following estimate:

$$
\begin{aligned}
& \left|\kappa \int_{s-\frac{1}{\kappa}}^{s} \int_{Q} \eta(u) \varphi(s) \varrho \mathrm{d} x \mathrm{~d} t-\int_{Q} \eta(u(s)) \varphi(s) \varrho \mathrm{d} x\right| \\
& =\left|\kappa \int_{s-\frac{1}{\kappa}}^{s} \int_{Q}(\eta(u)-\eta(u(s))) \varphi(s) \varrho \mathrm{d} x \mathrm{~d} t\right| \\
& \lesssim\left\|\eta^{\prime}\right\|_{\infty}\left|\kappa \int_{s-\frac{1}{\kappa}}^{s} \int_{Q}\right| u-u(s)|\varphi(s) \varrho \mathrm{d} x \mathrm{~d} t|,
\end{aligned}
$$

where (passing to a subsequence) the latter term converges to zero $\mathbb{P}$-almost surely. This is true since, by Theorem 3.3, $u$ is continuous in $L_{\omega}^{1} L_{w ; x}^{1}\left(\right.$ thus $\kappa \int_{s-\frac{1}{\kappa}}^{s}\left(\mathbb{E} \int_{Q} \mid u-\right.$ $u(s) \mid w \mathrm{~d} x) \mathrm{d} t \rightarrow 0), w$ is strictly positive in $Q$, and $\phi(s)$ has compact support in $Q$.

Taking $\kappa \rightarrow \infty$ in (6.2) (passing to a suitable subsequence) implies that $u$ is an entropy solution on $[s, T]$. Finally, by uniqueness of entropy solutions and the continuity in $L_{\omega}^{1} L_{w ; x}^{1}$, we conclude that $u(t)$ coincides with $u_{s}(t ; u(s ; \xi))$ in $L_{\omega}^{1} L_{w ; x}^{1}$ for every $t \in[s, T]$, which completes the proof.

Proof of Proposition 3.10. To prove that the map $P_{t}$ is a semigroup we need to show that $P_{t+s}=P_{s} P_{t}$ for every $0<s \leq t$. The argument follows [DPZ14, Proof of Theorem 9.14]. In particular, we prove that

$$
\mathbb{E}\left(F(u(t+s ; \xi)) \mid \mathcal{F}_{s}\right)=\left.\mathbb{E} F\left(u_{s}(t ; \zeta)\right)\right|_{\zeta=u(s ; \xi)}
$$

$\mathbb{P}$-almost surely for every $\xi \in L_{w ; x}^{1}$ and $F \in C_{b}\left(L_{w ; x}^{1}\right)$ (space of bounded continuous functions from $L_{w, x}^{1}$ to $\mathbb{R}$ ), which implies the result by virtue of the monotone class theorem.

We first consider $\xi \in L_{x}^{m+1}$. By Corollary 6.1 we have that the random variables $u(s+t ; \xi)$ and $u_{s}(t ; u(s ; \xi))$ coincide in $L_{\omega}^{1} L_{w ; x}^{1}$. Since $u(s ; \xi)$ is $\mathcal{F}_{s}$-measurable and $u(s ; \xi) \in L_{\omega}^{m+1} L_{x}^{m+1}$ (by the same argument as in the proof of Corollary 6.1), it suffices to prove that

$$
\mathbb{E}\left(F\left(u_{s}\left(t ; \zeta_{0}\right)\right) \mid \mathcal{F}_{s}\right)=\left.\mathbb{E} F\left(u_{s}(t ; \zeta)\right)\right|_{\zeta=\zeta_{0}},
$$

$\mathbb{P}$-almost surely, for every $\mathcal{F}_{s}$-measurable $\zeta_{0} \in L_{\omega}^{m+1} L_{x}^{m+1}$. If $\zeta_{0}$ is a simple random variable, (6.4) follows easily. Otherwise, there exists a sequence of simple random variables $\left(\zeta_{0}^{(n)}\right)_{n \geq 1}$, such that $\zeta_{0}^{(n)} \rightarrow \zeta_{0}$ in $L_{\omega}^{m+1} L_{x}^{m+1}$ as $n \rightarrow \infty$, which, in turn, implies that $\zeta_{0}^{(n)} \rightarrow \zeta_{0}$ in $L_{\omega}^{1} L_{w ; x}^{1}$. By (6.4) we know that for every $n \geq 1$,

$$
\mathbb{E}\left(F\left(u_{s}\left(t ; \zeta_{0}^{(n)}\right)\right) \mid \mathcal{F}_{s}\right)=\left.\mathbb{E} F\left(u_{s}(t ; \zeta)\right)\right|_{\zeta=\zeta_{0}^{(n)}} .
$$

Using (3.2) (with $u$ replaced by $u_{s}$ ) we obtain the estimate

$$
\mathbb{E}\left\|u_{s}\left(t ; \zeta_{0}^{(n)}\right)-u_{s}\left(t ; \zeta_{0}\right)\right\|_{L_{w ; x}^{1}} \leq \mathbb{E}\left\|\zeta_{0}^{(n)}-\zeta_{0}\right\|_{L_{w ; x}^{1},}
$$

which implies that $u_{s}\left(t ; \zeta_{0}^{(n)}\right) \rightarrow u_{s}\left(t ; \zeta_{0}\right)$ in $L_{\omega}^{1} L_{w ; x}^{1}$. Since $F$ is continuous and bounded, the left-hand side of $(6.5)$ converges to $\mathbb{E}\left(F\left(u_{s}\left(t ; \zeta_{0}\right)\right) \mid \mathcal{F}_{s}\right) \mathbb{P}$-almost surely passing to a subsequence. On the other hand, the mapping $L_{w ; x}^{1} \ni \zeta \mapsto \mathbb{E} F\left(u_{s}(t ; \zeta)\right)$ is continuous. Indeed, for arbitrary $\zeta \in L_{w ; x}^{1}$, let $\left(\zeta_{n}\right)_{n \geq 1}$ be a sequence in $L_{w ; x}^{1}$ such 
that $\zeta_{n} \rightarrow \zeta$ in $L_{w ; x}^{1}$. Then, for every subsequence $k_{n} \rightarrow \infty$ we can use (3.2) (with $u$ replaced by $u_{s}$ ) and the continuity and boundedness of $F$ to find a further subsequence $m_{k_{n}} \rightarrow \infty$ such that $\mathbb{E} F\left(u_{s}\left(t ; \zeta_{m_{k_{n}}}\right)\right) \rightarrow \mathbb{E} F\left(u_{s}(t ; \zeta)\right)$. Using the continuity of $L_{w ; x}^{1} \ni$ $\zeta \mapsto \mathbb{E} F\left(u_{s}(t ; \zeta)\right)$ and the fact that $\zeta_{0}^{(n)} \rightarrow \zeta_{0}$ in $L_{\omega}^{1} L_{w ; x}^{1}$, we conclude that the righthand side of (6.5) converges to $\left.\mathbb{E} F\left(u_{s}(t ; \zeta)\right)\right|_{\zeta=\zeta_{0}} \mathbb{P}$-almost surely. Hence, we can pass to the limit in (6.5) to obtain (6.4) for arbitrary $\mathcal{F}_{s}$-measurable $\zeta_{0} \in L_{\omega}^{m+1} L_{x}^{m+1}$. This proves (6.3) for $\xi \in L_{x}^{m+1}$.

Finally, if $\xi \in L_{w, x}^{1}$, we take any sequence $\xi_{n} \rightarrow \xi$ in $L_{w ; x}^{1}$ such that $\xi_{n} \in$ $L_{x}^{m+1}$. Using the same arguments as in the previous paragraph we obtain that $\mathbb{E}\left(F\left(u\left(t+s ; \xi_{n}\right)\right) \mid \mathcal{F}_{s}\right) \rightarrow \mathbb{E}\left(F(u(t+s ; \xi)) \mid \mathcal{F}_{s}\right) \mathbb{P}$-almost surely (upon relabeling a subsequence) and that the mapping $L_{w ; x}^{1} \ni \zeta \mapsto \mathbb{E} F\left(u_{s}(t ; \zeta)\right)$ is continuous. This implies (6.3) for $\xi \in L_{w, x}^{1}$.

To prove that the semigroup $P_{t}$ is Feller we simply note that if $\xi_{n} \rightarrow \xi$ in $L_{w ; x}^{1}$, then, by (3.2) for every subsequence $k_{n} \rightarrow \infty$, there exists a further subsequence $m_{k_{n}} \rightarrow \infty$ such that $u\left(t ; \xi_{m_{k_{n}}}\right) \rightarrow u(t ; \xi)$ in $L_{w ; x}^{1} \mathbb{P}$-almost surely. Then, by the continuity and boundedness of $F, P_{t} F\left(\xi_{m_{k_{n}}}\right) \rightarrow P_{t} F(\xi)$.

We are now ready to prove Theorem 3.12, following [Röc07, Proof of Theorem 4.3.9, Proof of Lemma 4.3.11].

Proof of Theorem 3.12. Step 1 . For $\xi \in L_{x}^{m+1}$ we let $\eta_{s}(\xi):=u_{s}(0 ; \xi)$. By Corollary 6.1 , for every $s_{1} \leq s_{2} \leq-1$ we have that

$$
\eta_{s_{1}}(\xi)=u_{s_{2}}\left(0 ; u_{s_{1}}\left(s_{2} ; \xi\right)\right)
$$

in $L_{\omega}^{1} L_{w ; x}^{1}$. By Theorem 3.8 we know that

$$
\mathbb{E}\left\|\eta_{s_{2}}(\xi)-\eta_{s_{1}}(\xi)\right\|_{L_{w ; x}^{1}} \lesssim\|w\|_{L_{x}^{m *}}^{m_{*}}\left|s_{2}\right|^{-\frac{1}{m-1}}
$$

The last inequality implies that $\left(\eta_{s}(\xi)\right)_{s \leq-1}$ is a Cauchy sequence in $L_{\omega}^{1} L_{w ; x}^{1}$. Hence there exists a random variable $\eta(\xi) \in L_{\omega}^{1} L_{w ; x}^{1}$ such that $\eta_{s}(\xi) \rightarrow \eta(\xi)$ as $s \rightarrow-\infty$.

We claim that $\eta(\xi)$ is independent of $\xi$. Indeed, again using Theorem 3.8 we have that for any $\xi, \tilde{\xi} \in L_{x}^{m+1}$,

$$
\mathbb{E}\left\|\eta_{s}(\xi)-\eta_{s}(\tilde{\xi})\right\|_{L_{w ; x}^{1}} \lesssim\|w\|_{L_{x}^{m_{*}}}^{m_{*}}|s|^{-\frac{1}{m-1}},
$$

and letting $s \rightarrow-\infty$ asserts our claim.

We now let $\mu=\mathcal{L}(\eta) \in \mathcal{M}_{1}\left(L_{w ; x}^{1}\right)$ for $\eta=\eta(0)$. Then $\mu \in \mathcal{M}_{1}\left(L_{x}^{m+1}\right)$ since

$$
\mathbb{E}\|\eta\|_{L_{x}^{m+1}}^{m+1} \leq \liminf _{s \rightarrow-\infty} \mathbb{E}\left\|\eta_{s}(0)\right\|_{L_{x}^{m+1}}^{m+1} \leq \sup _{s \leq-1} \mathbb{E}\left\|\eta_{s}(0)\right\|_{L_{x}^{m+1}}^{m+1}
$$

and the last quantity is bounded by Proposition 3.6. Similarly to Definition 3.9 we denote by $P_{s, t}$ the semigroup associated to (6.1) at time $t$. In this notation $P_{t}=P_{0, t}$. Then one has the following elementary calculation:

$$
\begin{aligned}
\int_{L_{w ; x}^{1}} P_{0, t} F(\xi) \mu(\mathrm{d} \xi) & =\lim _{s \rightarrow \infty} P_{-s, 0}\left(P_{0, t} F\right)(0) \\
& =\lim _{s \rightarrow \infty} P_{-s, t} F(0)=\lim _{s \rightarrow \infty} P_{-(t+s), 0} F(0)=\int_{L_{w ; x}^{1}} F(\xi) \mu(\mathrm{d} \xi)
\end{aligned}
$$

for every $F \in C_{b}\left(L_{w ; x}^{1}\right)$ (bounded continuous functions from $L_{w, x}^{1}$ to $\mathbb{R}$ ), where we also use the fact that $P_{s, t}$ is Feller for every $s<t$ and use as well the identities 
$P_{s, t}=P_{s+\tau, t+\tau}$ for every $\tau \in \mathbb{R}$ and $P_{s, \tau} P_{\tau, t}=P_{s, t}$ for every $s<\tau<t$. This implies that $P_{t}^{*} \mu=\mu$ as measures on $L_{w ; x}^{1}$ and hence on $L_{x}^{m+1}$ since both $P_{t}^{*} \mu$ and $\mu$ are supported on $L_{x}^{m+1}$.

Step 2. By Theorem 3.8, for every $F \in \operatorname{Lip}\left(L_{w ; x}^{1}\right)$ and $\xi, \tilde{\xi} \in L_{w ; x}^{1}$ we have that

$$
\left|P_{t} F(\xi)-P_{t} F(\tilde{\xi})\right| \lesssim\|F\|_{\operatorname{Lip}\left(L_{w ; x}^{1}\right)}\|w\|_{L_{x}^{m *}}^{m_{*}} t^{-\frac{1}{m-1}},
$$

which implies that any two invariant measures $\mu$ and $\tilde{\mu}$ on $L_{w ; x}^{1}$ coincide. Using the last estimate we also see that

$$
\left|P_{t} F(\xi)-\int_{L_{w ; x}^{1}} F(\tilde{\xi}) \mu(\mathrm{d} \tilde{\xi})\right| \lesssim\|F\|_{\operatorname{Lip}\left(L_{w ; x}^{1}\right)}\|w\|_{L_{x}^{m *}}^{m_{*}} t^{-\frac{1}{m-1}},
$$

which completes the proof by taking the supremum over $\|F\|_{\operatorname{Lip}\left(L_{w ; x}^{1}\right)} \leq 1$ and $\xi \in$ $L_{w ; x}^{1}$.

Appendix A. In this appendix we prove Proposition 5.5 using Galerkin approximations as in [DG18]. We also prove Lemma A.1, which is used in the proof of Proposition 3.6.

Proof of Proposition 5.5. We fix $n \geq 1$, and since $n$ is fixed, in order to ease the notation we drop the dependence on $n$ and relabel $\bar{A}:=A_{n}, \bar{\sigma}=\sigma_{n}, \bar{\xi}:=\xi_{n}$, and $u:=$ $u_{n}$. Let $\left(e_{k}\right)_{k=1}^{\infty} \subset H_{x}^{4} \cap H_{0, x}^{2}$ be an orthonormal basis of $L_{x}^{2}$ consisting of eigenvectors of $\Delta^{2}$. For $m=1,2$ let us denote by $H_{x}^{-m}$ the dual of $H_{0 ; x}^{m}$ equipped with the inner product $(\cdot, \cdot)_{H_{x}^{-m}}:=\left(\Delta^{-m} \cdot \Delta^{-m} \cdot\right)_{H_{0 ; x}^{m}}$, and let $\Pi_{l}: H_{x}^{-1} \rightarrow V_{l}:=\operatorname{span}\left\{e_{1}, \ldots, e_{l}\right\}$ be the projection operator, that is, for $v \in H_{x}^{-1}$,

$$
\Pi_{l} v:=\sum_{i=1}^{l} H^{-1}\left\langle v, e_{i}\right\rangle_{H_{0 ; x}^{1}} e_{i}
$$

Then, the Galerkin approximation

$$
\left\{\begin{array}{l}
d u_{l}=\Pi_{l} \Delta \bar{A}\left(u_{l}\right) \mathrm{d} t+\Pi_{l} \bar{\sigma}^{k}\left(u_{l}\right) \mathrm{d} \beta^{k}(t) \\
u(0)=\Pi_{l} \bar{\xi}
\end{array}\right.
$$

is an equation on $V_{l}$ with locally Lipschitz continuous coefficients having linear growth. Consequently, it admits a unique solution $u_{l}$, for which we have

$$
u_{l} \in L_{\omega, t}^{2} H_{0 ; x}^{1} \cap L_{\omega}^{2} C\left([0, T] ; L_{x}^{2}\right) .
$$

After applying Itô's formula for the function $u \mapsto\|u\|_{L_{x}^{2}}^{2}$, by standard arguments we obtain that

$$
\mathbb{E} \int_{0}^{T}\left\|u_{l}\right\|_{H_{0 ; x}^{1}}^{2} \mathrm{~d} t \leq C\left(1+\mathbb{E}\|\bar{\xi}\|_{L_{x}^{2}}^{2}\right)
$$

and that for every $p \geq 2$,

$$
\mathbb{E} \sup _{t \leq T}\left\|u_{l}(t)\right\|_{L_{x}^{2}}^{p} \leq C\left(1+\mathbb{E}\|\bar{\xi}\|_{L_{x}^{2}}^{p}\right)
$$

Copyright $@$ by SIAM. Unauthorized reproduction of this article is prohibited. 
where $C$ is independent of $l$. In $H_{x}^{-1}$ we have $\mathbb{P}$-almost surely for every $t \in[0, T]$,

$$
u_{l}(t)=\Pi_{l} \bar{\xi}+\int_{0}^{t} \Pi_{l} \Delta \bar{A}\left(u_{l}\right) \mathrm{d} s+\int_{0}^{t} \Pi_{l} \bar{\sigma}^{k}\left(u_{l}\right) \mathrm{d} \beta^{k}(s)=J_{l}^{1}+J_{l}^{2}(t)+J_{l}^{3}(t) .
$$

By Sobolev's embedding theorem and (A.2),

$$
\sup _{l} \mathbb{E}\left\|J_{l}^{2}\right\|_{W_{t}^{\frac{1}{3}, 4} H_{x}^{-1}}^{2} \leq \sup _{l} \mathbb{E}\left\|J_{l}^{2}\right\|_{H_{t}^{1} H_{x}^{-1}}^{2}<\infty .
$$

By [FG95, Lemma 2.1], the linear growth of $\sigma$, and (A.3) we have

$$
\sup _{l} \mathbb{E}\left\|J_{l}^{3}\right\|_{W_{t}^{\alpha, p} H_{x}^{-1}}^{p}<\infty
$$

for every $\alpha \in\left(0, \frac{1}{2}\right)$ and $p \geq 2$. By the last two estimates and (A.2) we obtain

$$
\sup _{l} \mathbb{E}\left\|u_{l}\right\|_{W_{t}^{\frac{1}{3}, 4} H_{x}^{-1} \cap L_{t}^{2} H_{0 ; x}^{1}}<\infty .
$$

By virtue of [FG95, Theorems 2.1 and 2.2] one can easily see that the embedding

$$
W_{t}^{\frac{1}{3}, 4} H_{x}^{-1} \cap L_{t}^{2} H_{0 ; x}^{1} \hookrightarrow L_{t}^{2} L_{x}^{2} \cap C\left([0, T] ; H_{x}^{-2}\right)=: \mathcal{X}
$$

is compact. It follows that for any sequences $\left(l_{q}\right)_{q \geq 1},\left(\bar{l}_{q}\right)_{q \geq 1}$, the laws of $\left(u_{l_{q}}, u_{\bar{l}_{q}}\right)_{q \geq 1}$ are tight on $\mathcal{X} \times \mathcal{X}$. Let us set

$$
\beta(t)=\sum_{k=1}^{\infty} \frac{1}{\sqrt{2^{k}}} \beta^{k}(t) \mathfrak{e}_{k},
$$

where $\left(\mathfrak{e}_{k}\right)_{k=1}^{\infty}$ is the standard orthonormal basis of $\ell^{2}$. By Prokhorov's theorem, there exists a (non-relabeled) subsequence $\left(u_{l_{q}}, u_{\bar{l}_{q}}\right)_{q \geq 1}$ such that the laws of $\left(u_{l_{q}}, u_{\bar{l}_{q}}, \beta\right)_{q \geq 1}$ on $\mathcal{Z}:=\mathcal{X} \times \mathcal{X} \times C\left([0, T] ; \ell^{2}\right)$ are weakly convergent. By Skorohod's representation theorem, there exist $\mathcal{Z}$-valued random variables $(\hat{u}, \check{u}, \tilde{\beta}),\left(\widehat{u_{l_{q}}}, \widetilde{u_{\overline{l_{q}}}}, \tilde{\beta}_{q}\right), q \geq 1$, on a probability space $(\tilde{\Omega}, \tilde{\mathcal{F}}, \tilde{\mathbb{P}})$ such that in $\mathcal{Z}, \tilde{\mathbb{P}}$-almost surely,

$$
\left(\widehat{u_{l_{q}}}, \widetilde{u_{\bar{l}_{q}}}, \tilde{\beta}_{q}\right) \rightarrow(\hat{u}, \check{u}, \tilde{\beta})
$$

as $l \rightarrow \infty$, and for each $q \geq 1$, as random variables in $\mathcal{Z}$,

$$
\left(\widehat{u_{l_{q}}}, \widetilde{u_{\bar{l}_{q}}}, \tilde{\beta}_{q}\right) \stackrel{d}{=}\left(u_{l_{q}}, u_{\bar{l}_{q}}, \beta\right) .
$$

Moreover, upon passing to a non-relabeled subsequence, we may assume that

$$
\left(\widehat{u_{l_{q}}}, \widetilde{u_{\bar{l}_{q}}}\right) \rightarrow(\hat{u}, \check{u})
$$

for almost every $(\tilde{\omega}, t, x)$. Let $\left(\tilde{\mathcal{F}}_{t}\right)_{t \in[0, T]}$ be the augmented filtration of $\mathcal{G}_{t}:=\sigma(\hat{u}(s)$, $\check{u}(s), \tilde{\beta}(s) ; s \leq t)$, and let $\tilde{\beta}^{k}(t):=\sqrt{2^{k}}\left(\tilde{\beta}(t), \mathfrak{e}_{k}\right)_{\ell^{2}}$. It is easy to see that $\tilde{\beta}^{k}, k \geq 1$, are independent, standard, real-valued $\tilde{\mathcal{F}}_{t}$-Wiener processes. Indeed, they are $\tilde{\mathcal{F}}_{t^{-}}$-adapted by definition, and they are independent since $\beta^{k}$ are. We only have to show that they are $\tilde{\mathcal{F}}_{t}$-Wiener processes. Let us fix $s<t$, and let $V$ be a bounded continuous function on $C\left([0, s] ; H_{x}^{-2}\right) \times C\left([0, s] ; H_{x}^{-2}\right) \times C\left([0, s] ; \ell^{2}\right)$. For each $l \geq 1$ we have

$$
\begin{aligned}
& \tilde{\mathbb{E}}\left(\tilde{\beta}_{q}^{k}(t)-\tilde{\beta}_{q}^{k}(s)\right) V\left(\left.\widehat{u_{l_{q}}}\right|_{[0, s]},{\widetilde{u_{\bar{l}}}}_{\bar{l}}{ }_{[0, s]},\left.\tilde{\beta}_{q}\right|_{[0, s]}\right) \\
& =\mathbb{E}\left(\beta^{k}(t)-\beta^{k}(s)\right) V\left(\left.u_{l_{q}}\right|_{[0, s]},\left.u_{\bar{l}_{q}}\right|_{[0, s]},\left.\beta\right|_{[0, s]}\right)=0,
\end{aligned}
$$

Copyright $@$ by SIAM. Unauthorized reproduction of this article is prohibited. 
which, by using uniform integrability and passing to the limit $q \rightarrow \infty$, shows that $\tilde{\beta}^{k}(t)$ is a $\mathcal{G}_{t}$-martingale. Similarly, $\left|\tilde{\beta}^{k}(t)\right|^{2}-t$ is a $\mathcal{G}_{t}$-martingale. By continuity of $\tilde{\beta}^{k}(t)$ and $\left|\beta^{k}(t)\right|^{2}-t$ and the fact that their supremum in time is integrable in $\omega$, one can easily see that they are also $\tilde{\mathcal{F}}_{t}$-martingales. Hence, by Lévy's characterization theorem (see, e.g., [KS91, Theorem 3.16]) $\tilde{\beta}^{k}$ are $\tilde{\mathcal{F}}_{t}$-Wiener processes.

We now show that both $\hat{u}$ and $\check{u}$ satisfy the equation

$$
d v=\Delta \bar{A}(v) d t+\bar{\sigma}^{k}(x, v) \mathrm{d} \tilde{\beta}^{k}(t) .
$$

Note that due to (A.2), we have that $\hat{u} \in L_{\tilde{\omega}, t}^{2} H_{0 ; x}^{1}$. Let us set

$$
\begin{aligned}
& \hat{M}(t):=\hat{u}(t)-\hat{u}(0)-\int_{0}^{t} \Delta \bar{A}(\hat{u}) \mathrm{d} s, \\
& \hat{M}_{q}(t):=\widehat{u_{l_{q}}}(t)-\widehat{u_{l_{q}}}(0)-\int_{0}^{t} \Pi_{l_{q}} \Delta \bar{A}\left(\widehat{u_{l_{q}}}\right) \mathrm{d} s, \\
& M_{q}(t):=u_{l_{q}}(t)-u_{l_{q}}(0)-\int_{0}^{t} \Pi_{l_{q}} \Delta \bar{A}\left(u_{l_{q}}\right) \mathrm{d} s .
\end{aligned}
$$

We will show that for any $\phi \in H_{x}^{-2}$ and $k \geq 1$, the processes

$$
\begin{aligned}
\hat{M}^{1}(t) & :=(\hat{M}(t), \phi)_{H^{-2}}, \\
\hat{M}^{2}(t) & :=(\hat{M}(t), \phi)_{H^{-2}}^{2}-\int_{0}^{t} \sum_{k=1}^{\infty}\left|\left(\bar{\sigma}^{k}(\hat{u}), \phi\right)_{H^{-2}}\right|^{2} \mathrm{~d} s,
\end{aligned}
$$

and

$$
\hat{M}^{3, k}(t):=\tilde{\beta}^{k}(t)(\hat{M}(t), \phi)_{H_{x}^{-2}}-\int_{0}^{t}\left(\bar{\sigma}^{k}(\hat{u}), \phi\right)_{H^{-2}} \mathrm{~d} s
$$

are continuous $\tilde{\mathcal{F}}_{t}$-martingales. We first show that they are continuous $\mathcal{G}_{t}$-martingales. Assume for now that $\phi=\Delta^{2} \psi$, where $\psi \in V_{l_{q}}$. For, $i=1,2,3$, let us also define the processes $\hat{M}_{q}^{i}, M_{q}^{i}$ similarly to $\hat{M}^{i}$ but with $\hat{M}, \hat{u}, \bar{\sigma}^{k}(\cdot)$ replaced by $\hat{M}_{q}, \widehat{u_{q}}, \Pi_{l_{q}} \bar{\sigma}^{k}(\cdot)$ and $M_{q}, u_{l_{q}}, \Pi_{l_{q}} \sigma^{k}(\cdot)$, respectively. Let us fix $s<t$, and let $V$ be a bounded continuous function on $C\left([0, s] ; H_{x}^{-2}\right) \times C\left([0, s] ; \ell^{2}\right)$. We have that

$$
\left(M_{q}(t), \phi\right)_{H^{-2}}=\int_{0}^{t}\left(\Pi_{l_{q}} \bar{\sigma}^{k}\left(u_{l_{q}}\right), \phi\right)_{H_{x}^{-2}} \mathrm{~d} \beta^{k}(s) .
$$

It follows that $M_{q}^{i}$ are continuous $\mathcal{F}_{t}$-martingales. Hence,

$$
\mathbb{E} V\left(\left.u_{l_{q}}\right|_{[0, s]},\left.u_{\bar{l}_{q}}\right|_{[0, s]},\left.\beta\right|_{[0, s]}\right)\left(M_{q}^{i}(t)-M_{q}^{i}(s)\right)=0,
$$

which, combined with (A.5), gives

$$
\tilde{\mathbb{E}} V\left(\left.\widehat{u_{l_{q}}}\right|_{[0, s]},\left.\widetilde{u_{\bar{l}_{q}}}\right|_{[0, s]},\left.\tilde{\beta}_{q}\right|_{[0, s]}\right)\left(\hat{M}_{q}^{i}(t)-\hat{M}_{q}^{i}(s)\right)=0 .
$$

Next, note that

$$
\begin{aligned}
\tilde{\mathbb{E}} \int_{0}^{T}\left|\left(\Pi_{l_{q}} \Delta \bar{A}\left(\widehat{u_{l_{q}}}\right)-\Delta \bar{A}(\hat{u}), \phi\right)_{H_{x}^{-2}}\right| \mathrm{d} t & =\tilde{\mathbb{E}} \int_{0}^{T}\left|\left(\bar{A}\left(\widehat{u_{l_{q}}}\right)-\bar{A}(\hat{u}), \Delta \psi\right)_{L_{x}^{2}}\right| \mathrm{d} t \\
& \leq \tilde{\mathbb{E}} \int_{0}^{T}\left\|\hat{u}-\widehat{u_{l_{q}}}\right\|_{L_{x}^{2}} \rightarrow 0
\end{aligned}
$$

Copyright $@$ by SIAM. Unauthorized reproduction of this article is prohibited. 
where the convergence follows from (A.4) and the fact that $\left(\int_{0}^{T}\left\|\widehat{u_{q}}-\hat{u}\right\|_{L_{x}^{2}} \mathrm{~d} t\right)_{q \geq 1}$ is uniformly integrable on $\Omega$ (which, in turn, follows from (A.2)). Hence, by (A.8) and (A.4) we see that for each $t \in[0, T]$,

$$
\left(\hat{M}_{q}(t), \phi\right)_{H_{x}^{-2}} \rightarrow(\hat{M}(t), \phi)_{H_{x}^{-2}}
$$

in probability. Then, one can easily verify that $\hat{M}_{q}^{i}(t) \rightarrow \hat{M}^{i}(t)$ in probability. Moreover, for every $\phi \in H_{x}^{-2}$ and every $p \geq 2$, by (A.5) and (A.3), we have

$$
\begin{aligned}
\sup _{q} \tilde{\mathbb{E}}\left|\left(\hat{M}_{q}(t), \phi\right)_{H_{x}^{-2}}\right|^{p} & =\sup _{q} \mathbb{E}\left|\int_{0}^{t}\left(\Pi_{l_{q}} \bar{\sigma}^{k}\left(u_{l_{q}}\right), \phi\right)_{H_{x}^{-2}} \mathrm{~d} \beta^{k}(s)\right|^{p} \\
& \lesssim\|\phi\|_{H_{x}^{-2}}^{p} \mathbb{E}\left(1+\|\bar{\xi}\|_{L_{x}^{2}}^{p}\right) .
\end{aligned}
$$

From this, one easily deduces that for each $i=1,2,3$ and for $t \in[0, T], M_{q}^{i}(t)$ are uniformly integrable. Hence, we can pass to the limit in (A.7) to obtain

$$
\tilde{\mathbb{E}} V\left(\left.\hat{u}\right|_{[0, s]},\left.\check{u}\right|_{[0, s]},\left.\tilde{\beta}\right|_{[0, s]}\right)\left(\hat{M}^{i}(t)-\hat{M}^{i}(s)\right)=0 .
$$

In addition, using the continuity of $\hat{M}^{i}(t)$ in $\phi$, uniform integrability, and the fact that $\cup_{q} \Delta^{2} V_{l_{q}}$ is dense in $H_{x}^{-2}$, it follows that (A.10) holds also for every $\phi \in H_{x}^{-2}$. Hence, for every $\phi \in H_{x}^{-2}, \hat{M}^{i}$ are continuous $\mathcal{G}_{t}$-martingales having all moments finite. In particular, by Doob's maximal inequality, they are uniformly integrable (in $t$ ), which, combined with continuity (in $t$ ), implies that they are also $\tilde{\mathcal{F}}_{t}$-martingales. By [Hof13, Proposition A.1] we obtain that $\tilde{\mathbb{P}}$-almost surely for every $\phi \in H_{x}^{-2}$ and $t \in[0, T]$,

$$
(\hat{u}(t), \phi)_{H^{-2}}=(\hat{u}(0), \phi)_{H_{x}^{-2}}+\int_{0}^{t}(\Delta \bar{A}(\hat{u}), \phi)_{H_{x}^{-2}} \mathrm{~d} s+\int_{0}^{t}\left(\bar{\sigma}^{k}(\hat{u}), \phi\right)_{H_{x}^{-2}} \mathrm{~d} \tilde{\beta}^{k}(s) .
$$

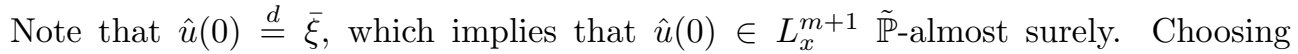
$\phi=\Delta^{2} \psi$ for $\psi \in C_{c}^{\infty}(Q)$, we obtain that for almost every $(\tilde{\omega}, t)$,

$$
(\hat{u}(t), \psi)_{L_{x}^{2}}=(\hat{u}(0), \psi)_{L_{x}^{2}}-\int_{0}^{t}(\nabla \bar{A}(\hat{u}), \nabla \psi)_{L_{x}^{2}} \mathrm{~d} s+\int_{0}^{t}\left(\bar{\sigma}^{k}(\hat{u}), \psi\right)_{L_{x}^{2}} \mathrm{~d} \tilde{\beta}^{k}(s) .
$$

It follows (see [KR79]) that $\hat{u}$ is a continuous $L_{x}^{2}$-valued $\tilde{\mathcal{F}}_{t^{-}}$-adapted process. Hence, $\hat{u}$ is an $L_{x}^{2}$-solution of $\mathcal{E}(\bar{A}, \hat{\sigma}, \hat{\xi})$ on $\left(\tilde{\Omega},\left(\tilde{\mathcal{F}}_{t}\right)_{t}, \tilde{\mathbb{P}}\right)$ with driving noise $\left(\tilde{\beta}^{k}\right)_{k=1}^{\infty}$, where $\hat{\xi}:=\hat{u}(0)$. Again, by standard arguments, for every $p \geq 2$ one has the estimate

$$
\mathbb{E} \sup _{t \leq T}\|\hat{u}(t)\|_{L_{x}^{p}}^{p}+\mathbb{E} \int_{0}^{T} \int_{Q}|\hat{u}|^{p-2}|\nabla \hat{u}|^{2} \mathrm{~d} x \mathrm{~d} t \leq N\left(1+\mathbb{E}\|\bar{\xi}\|_{L_{x}^{2}}^{p} .\right.
$$

Using this, Itô's formula (see, e.g., [Kry13]) for the function $u \mapsto \int_{Q} \eta(u) \varrho d x$, and Itô's product rule, one can see that $\hat{u}$ is an entropy solution of $\mathcal{E}(\bar{A}, \hat{\sigma}, \hat{\xi})$ on $\left(\tilde{\Omega},\left(\tilde{\mathcal{F}}_{t}\right)_{t}, \tilde{\mathbb{P}}\right)$ with driving noise $\left(\tilde{\beta}^{k}\right)_{k=1}^{\infty}$ and initial condition $\hat{\xi}:=\hat{u}(0)$. In the exact same way, $\check{u}$ is an $L_{x}^{2}$-solution and an entropy solution of $\mathcal{E}(\bar{A}, \bar{\sigma}, \tilde{\xi})$ (again, on $\left.\left(\tilde{\Omega},\left(\tilde{\mathcal{F}}_{t}\right)_{t}, \tilde{\mathbb{P}}\right)\right)$ with driving noise $\left(\tilde{\beta}^{k}\right)_{k=1}^{\infty}$ and $\check{\xi}:=\breve{u}(0)$. Furthermore, we have for $\delta>0$ 


$$
\begin{aligned}
\tilde{\mathbb{P}}\left(\|\hat{\xi}-\check{\xi}\|_{H_{x}^{-2}}>\delta\right) & \leq \delta^{-1} \tilde{\mathbb{E}}\|\hat{\xi}-\check{\xi}\|_{H_{x}^{-2}} \\
& \leq \liminf _{q \rightarrow \infty} \delta^{-1} \tilde{\mathbb{E}}\left\|\widehat{u_{q}}(0)-\widetilde{u_{\bar{l}_{q}}}(0)\right\|_{H_{x}^{-2}} \\
& =\liminf _{q \rightarrow \infty} \delta^{-1} \mathbb{E}\left\|\Pi_{l_{q}} \bar{\xi}-\Pi_{\bar{l}_{q}} \bar{\xi}\right\|_{H_{x}^{-2}}=0 .
\end{aligned}
$$

Hence, both $\hat{u}$ and $\check{u}$ are entropy solutions with the same initial condition. Moreover, by Lemma 5.3 they have the $(\star)$-property (4.1). Hence, by Corollary 4.5 we conclude that $\hat{u}=\breve{u}$. By [GK96, Lemma 1.1] we have that the initial sequence $\left(u_{l}\right)_{l=1}^{\infty}$ converges in probability in $\mathcal{X}$ to some $u \in \mathcal{X}$. Using this convergence and the uniform estimates on $u_{l}$, it is then straightforward to pass to the limit in (A.1) and see that $u$ is indeed an $L_{x}^{2}$-solution.

Lemma A.1. Let $u$ be an entropy solution of $\mathcal{E}(A, \sigma, \xi)$ for $A(r)=|r|^{m-1} r$. There exists $C>0$ depending only on $d, k, m$, and $|Q|$ and not on $T$ and $\xi$ such that

$$
\sup _{t \in[0, T]} \operatorname{ess}(t \wedge 1)^{\frac{m+1}{m-1}} \mathbb{E}\|u(t)\|_{L_{x}^{m+1}}^{m+1} \leq C .
$$

Proof. Let $u_{n}$ be the unique entropy solution of $\mathcal{E}\left(A_{n}, \sigma, \xi\right)$, where $A_{n}(r)=$ $|r|^{m-1} r+n^{-1} r$. It is easy to see by construction that $u_{n}$ is an $L_{x}^{2}$-solution (see Definition 5.2). By Itô's formula we see that

$$
\begin{aligned}
\mathbb{E}\left\|u_{n}(t)\right\|_{L_{x}^{m+1}}^{m+1}= & \mathbb{E}\|\xi\|_{L_{x}^{m+1}}^{m+1}-\mathbb{E} \int_{0}^{t}\left((m+1)\left\|\nabla u_{n}^{m}\right\|_{L_{x}^{2}}^{2}+\frac{(m+1) m}{2 n}\left\|\nabla u_{n}^{\frac{m+1}{2}}\right\|_{L_{x}^{2}}^{2}\right) \mathrm{d} s \\
& +\mathbb{E} \int_{0}^{t} \int_{Q} \frac{(m+1) m}{2}\left|u_{n}\right|^{m-1}\left|\sigma\left(u_{n}\right)\right|_{\ell^{2}}^{2} \mathrm{~d} x \mathrm{~d} s .
\end{aligned}
$$

By Poincaré's inequality and Jensen's inequality we have

$$
\left(\mathbb{E}\left\|u_{n}\right\|_{L_{x}^{m+1}}^{m+1}\right)^{\frac{2 m}{m+1}} \lesssim\left\|\nabla u_{n}^{m}\right\|_{L_{x}^{2}}^{2} .
$$

From this we obtain for $f(t):=\mathbb{E}\left\|u_{n}(t)\right\|_{L_{x}^{m+1}}^{m+1}$ the differential inequality

$$
f^{\prime}(t) \leq-C_{1}|f(t)|^{\frac{2 m}{m+1}}+C_{2} f(t)+C_{3},
$$

where for $i=1, \ldots, 3, C_{i}$ is a positive constant which depends on $d, K, m,|Q|$ but not on $T$. Using Young's inequality we obtain that

$$
f(t) \leq C\left(-|f(t)|^{\frac{2 m}{m+1}}+1\right),
$$

and by a simple comparison criterion (see [TW18, Lemma 3.8]),

$$
f(t) \leq C(t \wedge 1)^{-\frac{m+1}{m-1}}
$$

for some $C>0$ independent of $T$ and $f(0)$. The result follows if we let $n \rightarrow \infty$, since $u_{n} \rightarrow u$ in $L_{\omega, t}^{1} L_{w ; x}^{1}$.

\section{Appendix B.}

Lemma B.1. For every $m \geq 1$ there exists $C>0$ such that

$$
\left|\left(|u|^{m-1} u-|v|^{m-1} v\right)\right| \geq C|u-v|^{m}
$$

for every $u, v \in \mathbb{R}$. 
Proof. Let $f(z)=|z|^{m-1} z, z \in \mathbb{R}$. Without loss of generality, we can assume that $u-v>0$, which, by monotonicity, implies that $f(u)-f(v)=|u|^{m-1} u-|v|^{m-1} v$. We write

$$
f(u)-f(v)=\int_{v}^{u} \frac{\mathrm{d}}{\mathrm{d} z} f(z) \mathrm{d} z=m \int_{v}^{u}|z|^{m-1} \mathrm{~d} z .
$$

We distinguish among the cases $u, v \geq 0, u, v \leq 0$, and $u \geq 0 \geq v$.

Case $u, v \geq 0$. By a change of variables and the monotonicity of $\frac{\mathrm{d}}{\mathrm{d} z} f$ on $[0, \infty)$ we have that

$$
f(u)-f(v)=\int_{0}^{u-v} \frac{\mathrm{d}}{\mathrm{d} z} f(z+v) \mathrm{d} z \geq \int_{0}^{u-v} \frac{\mathrm{d}}{\mathrm{d} z} f(z) \mathrm{d} z=(u-v)^{m} .
$$

Case $u, v \leq 0$. By symmetry we get

$$
f(u)-f(v)=\int_{-u}^{-v} \frac{\mathrm{d}}{\mathrm{d} z} f(z) \mathrm{d} z .
$$

Then, similarly to the first case, we observe that

$$
f(u)-f(v)=\int_{0}^{-(v-u)} \frac{\mathrm{d}}{\mathrm{d} z} f(z-u) \mathrm{d} z \geq \int_{0}^{-(v-u)} f(z) \mathrm{d} z=(u-v)^{m} .
$$

Case $u \geq 0 \geq v$. We first assume that $-\frac{1}{2}(u-v) \leq v \leq 0$. In this case $u \geq \frac{1}{2}(u-v)$, and we get the bound

$$
f(u)-f(v) \geq \int_{0}^{\frac{1}{2}(u-v)} \frac{\mathrm{d}}{\mathrm{d} z} f(z) \mathrm{d} z=\frac{1}{2^{m}}(u-v)^{m} .
$$

We now assume that $v \leq-\frac{1}{2}(u-v)$. Then

$$
f(u)-f(v) \geq \int_{-\frac{1}{2}(u-v)}^{0} \frac{\mathrm{d}}{\mathrm{d} z} f(z) \mathrm{d} z=\frac{1}{2^{m}}(u-v)^{m} .
$$

The above assertions complete the proof.

Lemma B.2. Let $f, h: \mathbb{R} \rightarrow \mathbb{R}$ be continuous functions such that for every $s \leq t$,

$$
\left\{\begin{array}{l}
f(t)-f(s) \leq-C \int_{s}^{t}|f(\tau)|^{m-1} f(\tau) \mathrm{d} \tau \\
h(t)-h(s)=-C \int_{s}^{t}|h(\tau)|^{m-1} h(\tau) \mathrm{d} \tau
\end{array}\right.
$$

for some $C>0$. Then, provided $f(0) \leq h(0)$, we have that $f(t) \leq h(t)$ for every $t \geq 0$.

Proof. Let $h_{\varepsilon}(t)$ be the unique continuous solution of

$$
\left\{\begin{array}{l}
h_{\varepsilon}^{\prime}(t)=-C\left|h_{\varepsilon}(t)\right|^{m-1} h_{\varepsilon}(t)+\varepsilon \\
h_{\varepsilon}(0)=h(0)
\end{array}\right.
$$

We show that for every $t>0$ and $\varepsilon \in(0,1)$ we have that $f(t) \leq h_{\varepsilon}(t)$. The result is then immediate if we pass to the limit $\varepsilon \rightarrow 0$.

Copyright (C) by SIAM. Unauthorized reproduction of this article is prohibited. 
Assume for contradiction that there exists $t>0$ such that $f(t)>h_{\varepsilon}(t)$. Using the continuity of $f$ and $h_{\varepsilon}$ and the fact that $f(0) \leq h(0)$ we may assume that there exist $s<t_{*} \leq t$ such that $f(s)=h_{\varepsilon}(s)$ and $f(\tau)>h_{\varepsilon}(\tau)$ for every $\tau \in\left(s, t_{*}\right]$. This implies that for every $\tau \in\left(s, t_{*}\right]$,

$$
h_{\varepsilon}(\tau)-h_{\varepsilon}(s)<f(\tau)-f(s) \leq-C \int_{s}^{\tau}|f(\bar{\tau})|^{m-1} f(\bar{\tau}) \mathrm{d} \bar{\tau} .
$$

Multiplying the last inequality by $(\tau-s)^{-1}$ and passing to the limit $\tau \rightarrow s$, we obtain that

$$
-C\left|h_{\varepsilon}(s)\right|^{m-1} h_{\varepsilon}(s)+\varepsilon=h_{\varepsilon}^{\prime}(s) \leq-C|f(s)|^{m-1} f(s),
$$

which is a contradiction since $f(s)=h_{\varepsilon}(s)$. This completes the proof.

\section{REFERENCES}

[AP81]

[BDPR04]

[BDPR16]

[BGLR11]

[BR11]

[CCP19]

[DG18]

[DGG19]

[DPR04a]

[DPR04b]

[DPRRW06]

[DPZ14]

[FG95]

[FGS17]

[Ges13]

[Ges14]

[Ges15]

[GH18]
D. G. Aronson And L. A. Peletier, Large time behaviour of solutions of the porous medium equation in bounded domains, J. Differential Equations, 39 (1981), pp. $378-412$.

V. I. Bogachev, G. Da Prato, And M. Röckner, Invariant measures of generalized stochastic equations of porous media, Dokl. Akad. Nauk, 396 (2004), pp. 7-11 (in Russian).

V. Barbu, G. Da Prato, and M. Röckner, Stochastic Porous Media Equations, Lecture Notes in Math. 2163, Springer, Cham, 2016.

W.-J. Beyn, B. Gess, P. Lescot, AND M. RöCKNer, The global random attractor for a class of stochastic porous media equations, Comm. Partial Differential Equations, 36 (2011), pp. 446-469.

V. BARBU AND M. RöCKNER, On a random scaled porous media equation, J. Differential Equations, 251 (2011), pp. 2494-2514.

J. A. Carrillo, K. Craig, and F. S. Patacchini, A blob method for diffusion, Calc. Var. Partial Differential Equations, 58 (2019), 53.

K. Dareiotis And B. Gess, Nonlinear diffusion equations with nonlinear gradient noise, Electron. J. Probab., 25 (2020), 35.

K. Dareiotis, M. Gerencsér, And B. Gess, Entropy solutions for stochastic porous media equations, J. Differential Equations, 266 (2019), pp. 3732-3763.

G. DA PRATO AND M. RÖCKNeR, Invariant measures for a stochastic porous medium equation, in Stochastic Analysis and Related Topics in Kyoto, Adv. Stud. Pure Math. 41, Math. Soc. Japan, Tokyo, 2004, pp. 13-29.

G. Da Prato And M. RöckneR, Weak solutions to stochastic porous media equations, J. Evol. Equ., 4 (2004), pp. 249-271.

G. Da Prato, M. Röckner, B. L. Rozovskit, And F.-Y. WAng, Strong solutions of stochastic generalized porous media equations: Existence, uniqueness, and ergodicity, Comm. Partial Differential Equations, 31 (2006), pp. 277-291.

G. Da Prato and J. Zabczyk, Stochastic Equations in Infinite Dimensions, 2nd ed., Encyclopedia Math. Appl. 152, Cambridge University Press, Cambridge, UK, 2014.

F. Flandoli and D. GatareK, Martingale and stationary solutions for stochastic Navier-Stokes equations, Probab. Theory Related Fields, 102 (1995), pp. 367391.

F. Flandoli, B. Gess, and M. Scheutzow, Synchronization by noise for orderpreserving random dynamical systems, Ann. Probab., 45 (2017), pp. 1325-1350.

B. Gess, Random attractors for degenerate stochastic partial differential equations, J. Dynam. Differential Equations, 25 (2013), pp. 121-157.

B. Gess, Random attractors for stochastic porous media equations perturbed by space-time linear multiplicative noise, Ann. Probab., 42 (2014), pp. 818-864.

B. Gess, Finite time extinction for stochastic sign fast diffusion and self-organized criticality, Comm. Math. Phys., 335 (2015), pp. 309-344.

B. Gess and M. Hofmanová, Well-posedness and regularity for quasilinear degenerate parabolic-hyperbolic SPDE, Ann. Probab., 46 (2018), pp. 2495-2544. 
[GK96] I. GYÖNGY AND N. KRYLOv, Existence of strong solutions for Itô's stochastic equations via approximations, Probab. Theory Related Fields, 105 (1996), pp. 143158.

[GT14] B. GeSs ANd J. M. Tölle, Multi-valued, singular stochastic evolution inclusions, J. Math. Pures Appl. (9), 101 (2014), pp. 789-827.

[GT16] B. GESS AND J. M. TÖLLE, Ergodicity and local limits for stochastic local and nonlocal p-Laplace equations, SIAM J. Math. Anal., 48 (2016), pp. 4094-4125, https: //doi.org/10.1137/15M1049774.

[Hof13] M. Hofmanová, Degenerate parabolic stochastic partial differential equations, Stochastic Process. Appl., 123 (2013), pp. 4294-4336.

[KR79] N. V. KRYlov and B. L. Rozovskĭ,, Stochastic evolution equations, in Current Problems in Mathematics, Vol. 14, Akad. Nauk SSSR, Vsesoyuz. Inst. Nauchn. i Tekhn. Informatsii, Moscow, 1979, pp. 71-147, 256 (in Russian).

[Kry13] N. V. KRYLOV, A relatively short proof of Itô's formula for SPDEs and its applications, Stoch. Partial Differ. Equ. Anal. Comput., 1 (2013), pp. 152-174.

[KS91] I. Karatzas And S. E. Shreve, Brownian Motion and Stochastic Calculus, 2nd ed., Grad. Texts in Math. 113, Springer-Verlag, New York, 1991.

[Liu09] W. LiU, Harnack inequality and applications for stochastic evolution equations with monotone drifts, J. Evol. Equ., 9 (2009), pp. 747-770.

[LMG01] P.-L. Lions AND S. Mas-Gallic, Une méthode particulaire déterministe pour des équations diffusives non linéaires, C. R. Acad. Sci. Paris Sér. I Math., 332 (2001), pp. 369-376.

[Lot07] S. V. Lототsкy, A random change of variables and applications to the stochastic porous medium equation with multiplicative time noise, Commun. Stoch. Anal., 1 (2007), pp. 343-355.

[LR15] W. LiU And M. Röckner, Stochastic Partial Differential Equations: An Introduction, Universitext, Springer, Cham, 2015.

[MR93] S. MÉLÉARD AND S. RoELly, Interacting measure branching processes. Some bounds for the support, Stochastics Stochastics Rep., 44 (1993), pp. 103-121.

[Röc07] M. Röckner, Introduction to Stochastic Partial Differential Equations, Lecture Notes, Purdue University, 2007.

[TW18] P. Tsatsoulis AND H. WeBer, Spectral gap for the stochastic quantization equation on the 2-dimensional torus, Ann. Inst. Henri Poincaré Probab. Stat., 54 (2018), pp. 1204-1249.

[Wan07] F.-Y. WANG, Harnack inequality and applications for stochastic generalized porous media equations, Ann. Probab., 35 (2007), pp. 1333-1350.

[Wan13] F.-Y. WANG, Harnack Inequalities for Stochastic Partial Differential Equations, SpringerBriefs in Math., Springer, New York, 2013.

[Wan15a] F.-Y. WANG, Exponential convergence of non-linear monotone SPDEs, Discrete Contin. Dyn. Syst., 35 (2015), pp. 5239-5253.

[Wan15b] F.-Y. WANG, Asymptotic couplings by reflection and applications for nonlinear monotone SPDES, Nonlinear Anal., 117 (2015), pp. 169-188.

Copyright $@$ by SIAM. Unauthorized reproduction of this article is prohibited. 